\begin{tabular}{|c|c|c|c|c|c|c|}
\hline \multirow{4}{*}{ Impact Factor: } & ISRA (India) & $=3.117$ & SIS (USA) & $=0.912$ & ICV (Poland) & $=6.630$ \\
\hline & ISI (Dubai, UAE & $=0.829$ & РИНЦ (Russia) & $=0.156$ & PIF (India) & $=1.940$ \\
\hline & GIF (Australia) & $=0.564$ & ESJI (KZ) & $=8.716$ & IBI (India) & $=4.260$ \\
\hline & JIF & $=1.500$ & SJIF (Morocco) & $=5.667$ & OAJI (USA) & $=0.350$ \\
\hline
\end{tabular}

\begin{tabular}{|c|c|}
\hline $\begin{array}{l}\text { SOI: } \underline{1.1 / \mathrm{T}} \\
\text { International Sc} \\
\text { Theoretical } \boldsymbol{\&}\end{array}$ & $\begin{array}{l}\frac{\mathrm{AS}}{\mathrm{D}} \text { DOI } 10.15863 / \mathrm{TAS} \\
\text { ientific Journal } \\
\text { Applied Science }\end{array}$ \\
\hline p-ISSN: 2308-4944 (print) & e-ISSN: 2409-0085 (online) \\
\hline Year: $2019 \quad$ Issue: 02 & Volume: 70 \\
\hline & http://T-Science.org \\
\hline
\end{tabular}

SECTION 7. Mechanics and machine construction.
QR - Issue
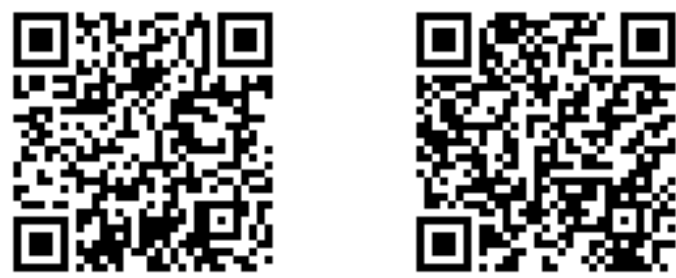

Denis Chemezov

M.Sc.Eng., Corresponding Member of International Academy of Theoretical and Applied Sciences, Lecturer of Vladimir Industrial College, Russian Federation https://orcid.org/0000-0002-2747-552X chemezov-da@yandex.ru

Anzhelika Bayakina Lecturer of Vladimir Industrial College, Russian Federation

Lyubov Suvorova Student of Vladimir Industrial College, Russian Federation

Irina Pavluhina Lecturer of Vladimir Industrial College, Russian Federation

Oleg Stepanov Student of Vladimir Industrial College, Russian Federation

Alexandra Strunina Lecturer of Vladimir Industrial College, Russian Federation

Ivan Mochalov Lecturer of Vladimir Industrial College, Russian Federation

Elena Kiseleva Master of Industrial Training, Vladimir Industrial College, Russian Federation

\title{
ISOSURFACES OF MECHANICAL STRESSES IN CANTILEVER AND DOUBLY SUPPORTED STEEL I-BEAMS SUBJECT TO BENDING
}

Abstract: Comparison of stress condition of cantilever and doubly supported steel I-beams after removing of external forces and moment was performed in the article. Stresses tensors are presented by color contours on a volume of deformed models of the I-beams. The most dangerous sections of the I-beams subject to bending at action of various external active loads were determined.

Key words: an I-beam, bending, stress, tensor, deformation, force, moment, a model, a component.

Language: English

Citation: Chemezov, D., et al. (2019). Isosurfaces of mechanical stresses in cantilever and doubly supported steel I-beams subject to bending. ISJ Theoretical \& Applied Science, 02 (70), 301-339.

Soi: http://s-o-i.org/1.1/TAS-02-70-30 Doi: crossef https://dx.doi.org/10.15863/TAS.2019.02.70.30 


\begin{tabular}{|c|c|c|c|c|c|c|}
\hline \multirow{4}{*}{ Impact Factor: } & ISRA (India) & $=3.117$ & SIS (USA) & $=0.912$ & ICV (Poland) & $=6.630$ \\
\hline & ISI (Dubai, UAE & $=0.829$ & РИНЦ (Russia & $=0.156$ & PIF (India) & $=1.940$ \\
\hline & GIF (Australia) & $=0.564$ & ESJI (KZ) & $=8.716$ & IBI (India) & $=4.260$ \\
\hline & JIF & $=1.500$ & SJIF (Morocco & $=5.667$ & OAJI (USA) & $=0.350$ \\
\hline
\end{tabular}

\section{Introduction}

I-beams are subjected to bending at action of external active forces and moments [1 -9]. A small value of load on the I-beam causes elastic deformations in material. Plastic deformation occurs with the value increasing of applied load. Plastic deformation of the steel I-beam leads to a shape changing of structural elements (flanges and a web), cracks development and subsequent partial destruction of material. Maximum stress occurs in a material volume at maximum deflection of the Ibeam and in a field of supports at different loading schemes. Stress in material can be presented by normal and tangential stresses that occur along three coordinate axes of the Cartesian coordinate system. Thus, complete volumetric presentation of stress condition of material at the calculation of stresses tensors is given. This will reveal the most loaded outer and inner layers of the I-beams material by means of constructed isosurfaces.

\section{Materials and methods}

Materials and methods of research of the computer experiments are presented in the work [10]. The research objects were models of the cantilever and doubly supported I-beams, on which external active forces (concentrated and distributed) and moments were applied.

\section{Results and discussion}

The models of four cantilever and five doubly supported I-beams were subjected to bending. The isosurfaces of mechanical stresses of the steel Ibeams after removing of concentrated moment (A and $\mathrm{E})$, concentrated force $(\mathrm{B}, \mathrm{C}, \mathrm{F}$ and $\mathrm{H})$ and distributed force $(D, G$ and $I)$ are presented in the Fig. 1 - 36. All models of the cantilever I-beams were rigidly fixed to a wall on the left side. All models of the doubly supported I-beams were mounted on the hinged immovable support (left) and the hinged movable support (right).

Stress visualization of the steel I-beam at bending is presented by Gauss-points. Directions of the first, second and third principal stresses of the deformed I-beams material are determined by the $x, y$ and $z$ coordinate axes in accordance with the coordinate system located in the lower left corner of each figure. Principal stresses directions of material of the cantilever I-beams were determined: the volumes of the bottom flange, the web on the right side and on the side of rigid restraint on all axes at action of concentrated moment clockwise; the volumes of the web and the beam flanges on the $y$ and $z$ axes at action of concentrated force on a loose end of the beam; complex stress condition, a right part of the beam has not subjected to stress (the $y$ - axis) at action of concentrated force on $1 / 2$ of the beam length; the volumes of the web and the flange below the neutral axis, the top flange at action of distributed force along the entire length of the beam. Principal stresses directions of material of the doubly supported I-beams were determined: the volumes of the top and bottom flanges, the part of the web at action of concentrated moment clockwise; complex stress condition at action of concentrated and distributed forces on $1 / 2$ of the beam length, distributed force along the entire length of the beam and concentrated force on $1 / 3$ of the beam length.

The first Piola-Kirchhoff stress tensor (asymmetric) is a material measure of stress in the deformed point of a solid. It is defined as the ratio of stress vector to an unit normal. Tensor is presented by the $x X-z Z$ components. The $x Y$ and $y X, x Z$ and $z X, y Z$ and $z Y$ components are the same by the value and distribution of the isosurfaces of the I-beam material stresses. The calculated stress value in the $z Z$ component is maximum of all nine components.

The deviatoric second Piola-Kirchhoff stress tensor (symmetric) is presented as shear stress tensor. Shear stress at bending of the cantilever and doubly supported I-beams is observed in the volumes of the flanges and the web in direction of the $X, Y, Z$ coordinate axes and the $Y Z$ coordinate plane. Stress in direction of the $X Y$ and $X Z$ coordinate planes occurs only in the flanges of the I-beams.

Stress tensor is second-rank tensor consisting of nine parameters (written as a matrix) presenting mechanical stresses at the arbitrary point of the loaded solid. Calculated stress tensor of the I-beams material consists of six components. The values and distribution of stresses in the $x$ component are identical to stresses in the $x X$ component of the first Piola-Kirchhoff stress tensor. The values and distribution of stresses in the $x y$ component are identical to stresses in the $x Y$ and $y X$ components of the first Piola-Kirchhoff stress tensor and in the $X Y$ component of the deviatoric second Piola-Kirchhoff stress tensor. The values and distribution of stresses in the $x z$ component are identical to stresses in the $x Z$ and $z X$ components of the first Piola-Kirchhoff stress tensor and in the $X Z$ component of the deviatoric second Piola-Kirchhoff stress tensor. The values and distribution of stresses in the $y$ component are identical to stresses in the $y Y$ component of the first Piola-Kirchhoff stress tensor. The values and distribution of stresses in the $y z$ component are identical to stresses in the $y Z$ and $z Y$ components of the first Piola-Kirchhoff stress tensor and in the $Y Z$ component of the deviatoric second Piola-Kirchhoff stress tensor. The values and distribution of stresses in the $z$ component are identical to stresses in the $z Z$ component of the first Piola-Kirchhoff stress tensor. 


\begin{tabular}{|c|c|c|c|c|c|c|}
\hline \multirow{4}{*}{ Impact Factor: } & ISRA (India) & $=3.117$ & SIS (USA) & $=0.912$ & ICV (Poland) & $=6.630$ \\
\hline & ISI (Dubai, UAE & $=0.829$ & РИНЦ (Russia & $=0.156$ & PIF (India) & $=1.940$ \\
\hline & GIF (Australia) & $=0.564$ & ESJI (KZ) & $=8.716$ & IBI (India) & $=4.260$ \\
\hline & JIF & $=1.500$ & SJIF (Morocco & $=5.667$ & OAJI (USA) & $=0.350$ \\
\hline
\end{tabular}

A

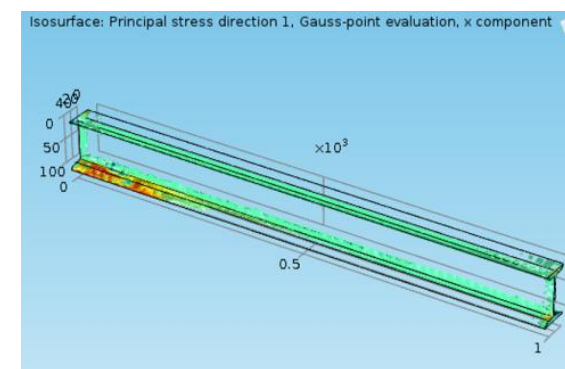

C

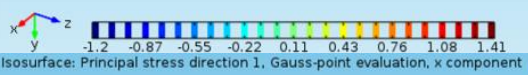

E

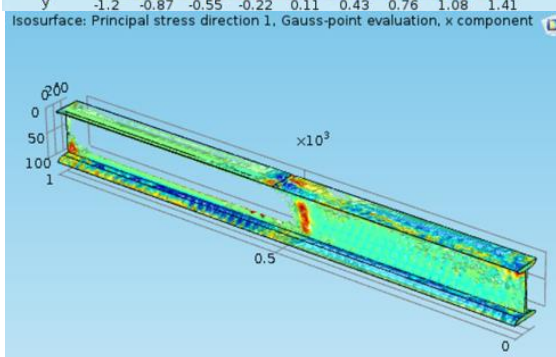

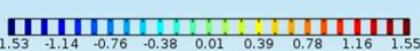
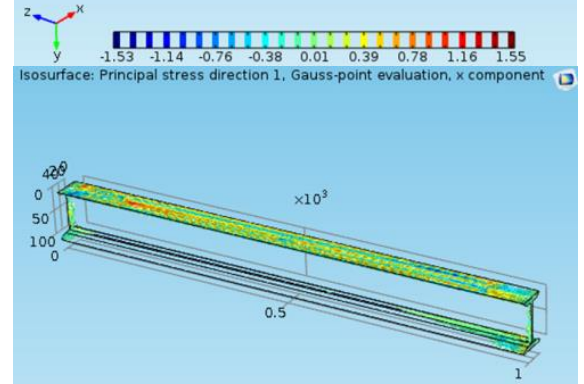

G

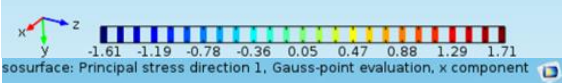

I

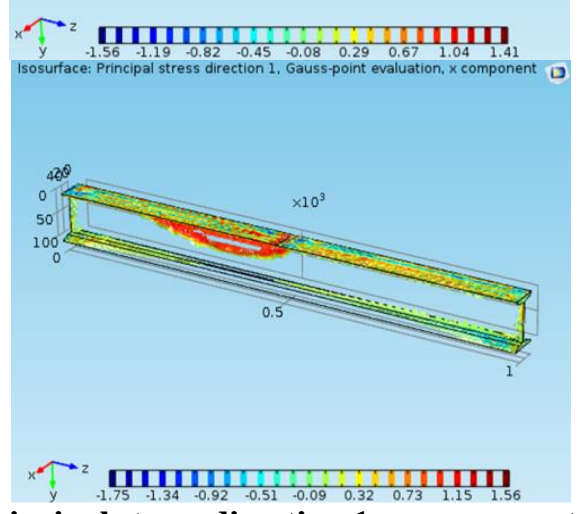

B

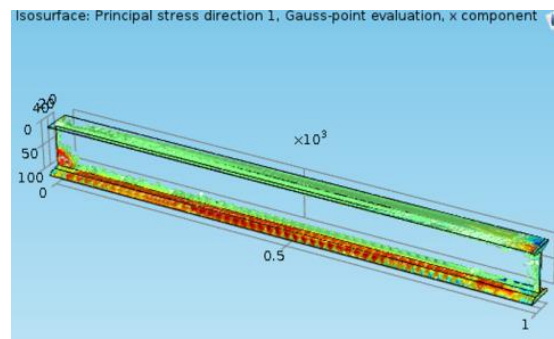

D

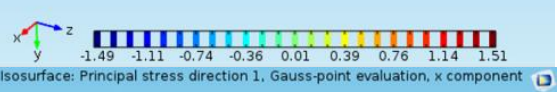

F

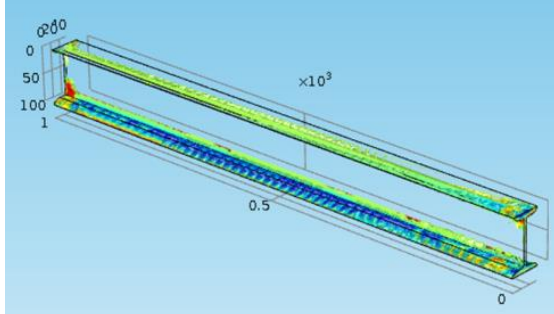

F

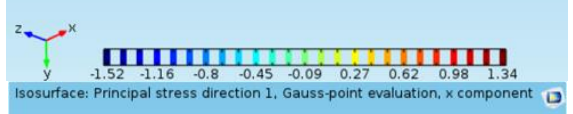

H
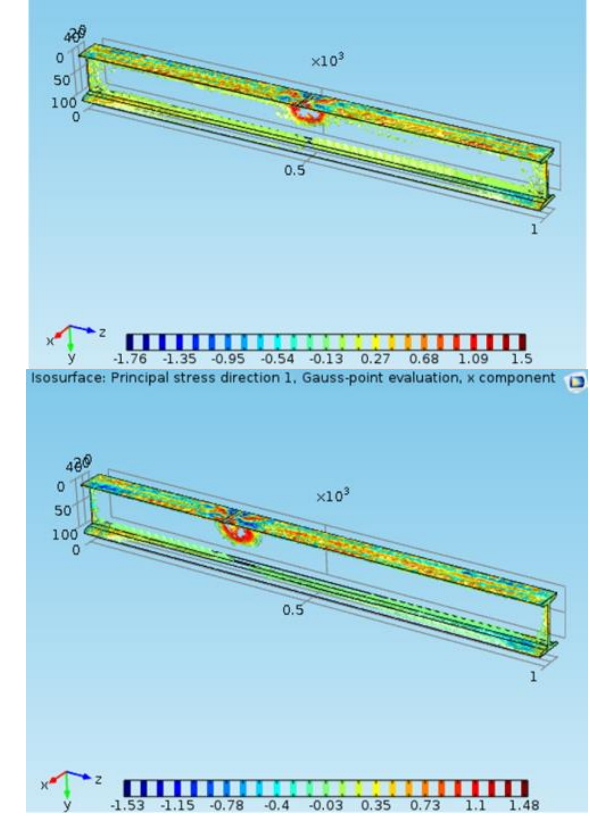

Figure 1 - Principal stress direction 1, $\mathrm{x}$ component: $\mathrm{A}$ - the first scheme; $\mathrm{B}$ - the second scheme; $\mathrm{C}$ - the third scheme; D - the fourth scheme; E - the fifth scheme; F - the sixth scheme; G - the seventh scheme; H the eighth scheme; I - the ninth scheme. 


\begin{tabular}{|c|c|c|c|c|c|c|}
\hline \multirow{4}{*}{ Impact Factor: } & ISRA (India) & $=3.117$ & SIS (USA) & $=0.912$ & ICV (Poland) & $=6.630$ \\
\hline & ISI (Dubai, UAE & $=0.829$ & РИНЦ (Russia & $=0.156$ & PIF (India) & $=1.940$ \\
\hline & GIF (Australia) & $=0.564$ & ESJI (KZ) & $=8.716$ & IBI (India) & $=4.260$ \\
\hline & JIF & $=1.500$ & SJIF (Morocco & $=5.667$ & OAJI (USA) & $=0.350$ \\
\hline
\end{tabular}

A

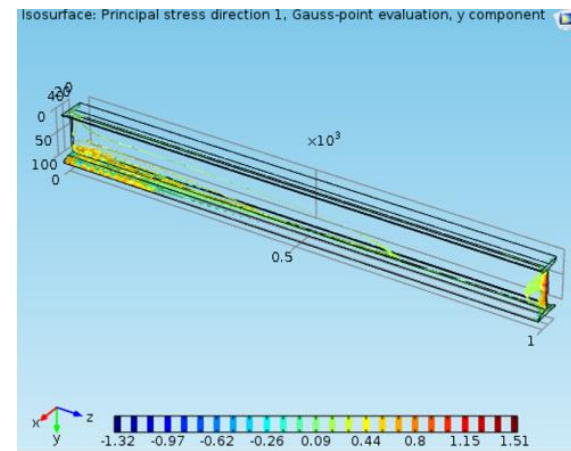

C

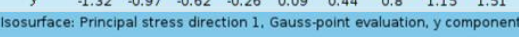

$\mathbf{E}$

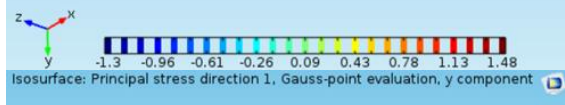

G
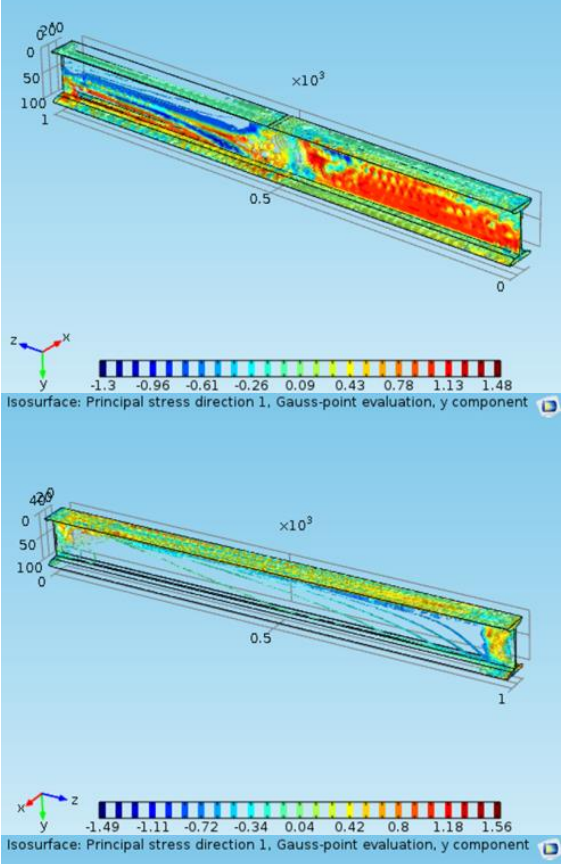

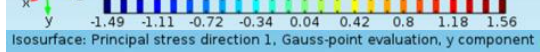

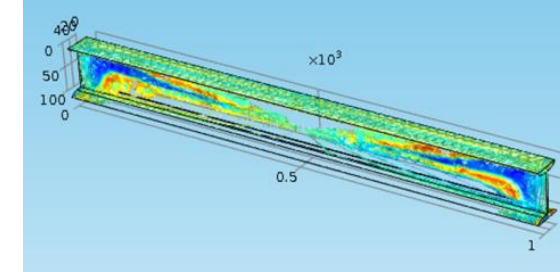

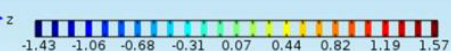

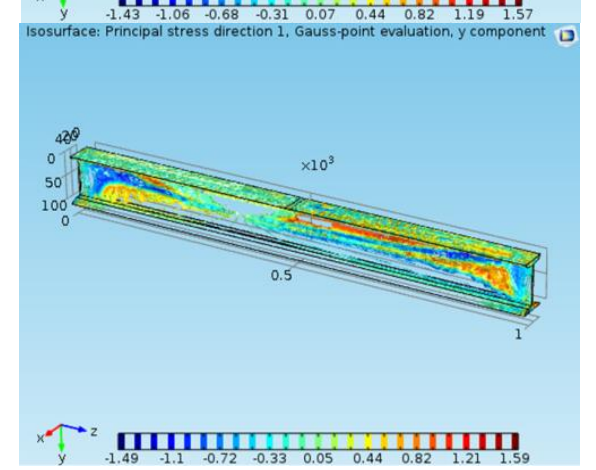

I
B

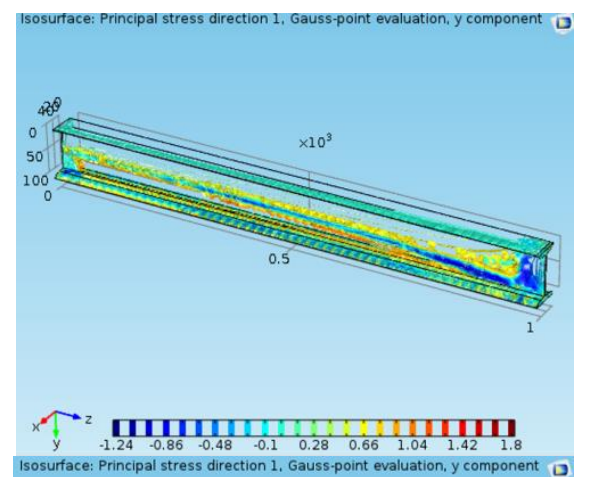

D

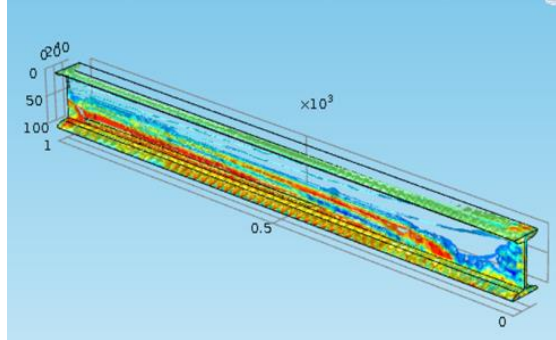

F

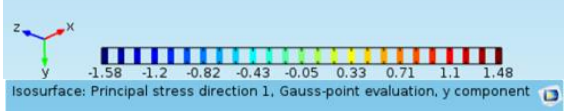

H
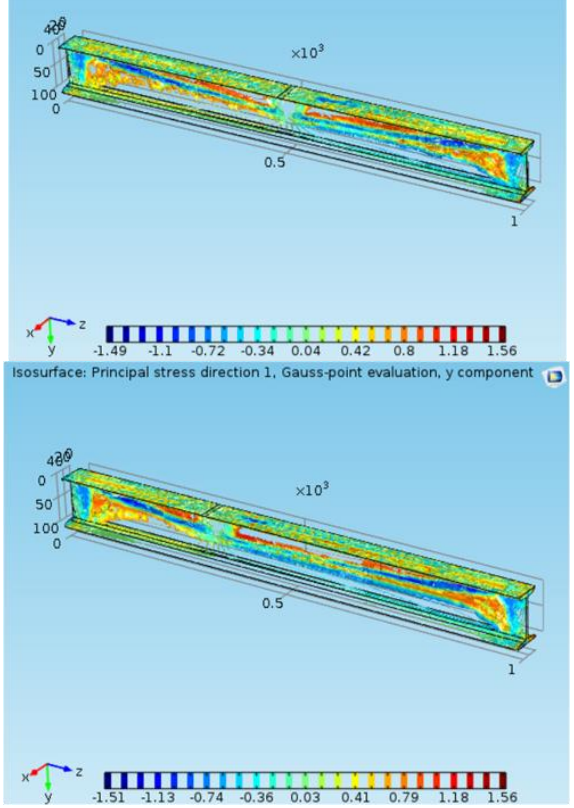

Figure 2 - Principal stress direction 1, y component: A - the first scheme; B - the second scheme; $\mathbf{C}$ - the third scheme; D - the fourth scheme; E - the fifth scheme; F - the sixth scheme; G - the seventh scheme; H the eighth scheme; I - the ninth scheme. 


\begin{tabular}{|c|c|c|c|c|c|c|}
\hline \multirow{4}{*}{ Impact Factor: } & ISRA (India) & $=3.117$ & SIS (USA) & $=0.912$ & ICV (Poland) & $=6.630$ \\
\hline & ISI (Dubai, UAE & $=0.829$ & РИНЦ (Russia & $=0.156$ & PIF (India) & $=1.940$ \\
\hline & GIF (Australia) & $=0.564$ & ESJI (KZ) & $=8.716$ & IBI (India) & $=4.260$ \\
\hline & JIF & $=1.500$ & SJIF (Morocco & $=5.667$ & OAJI (USA) & $=0.350$ \\
\hline
\end{tabular}

$\mathbf{A}$

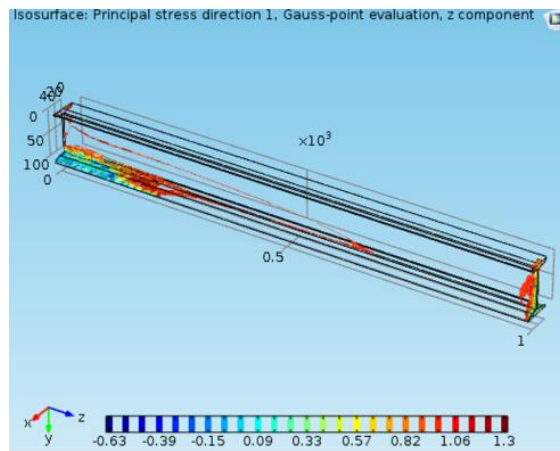

C

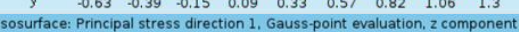

$\mathbf{E}$

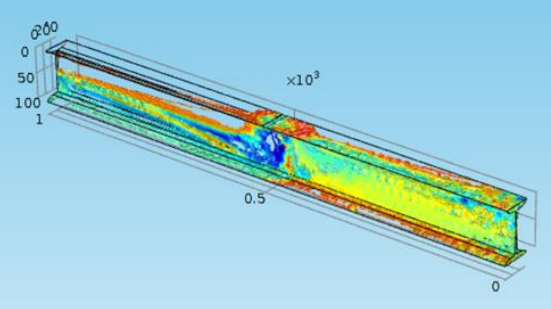

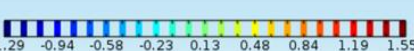

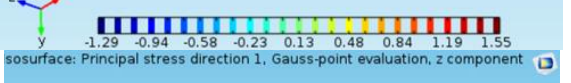

G
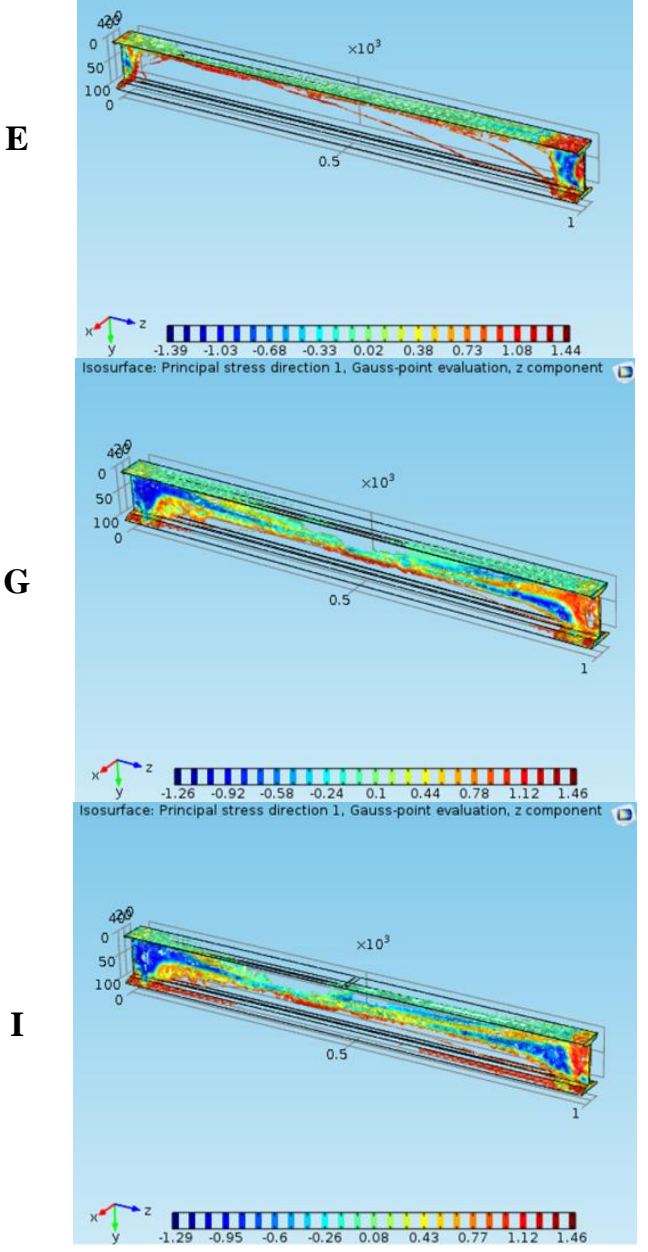

B

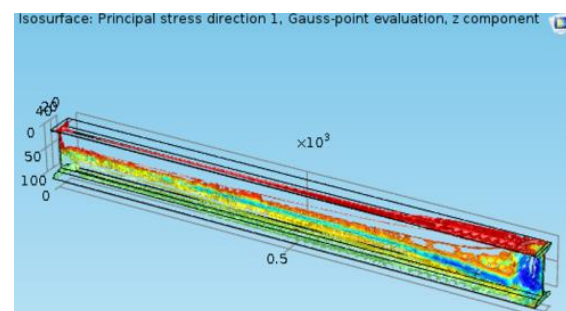

D
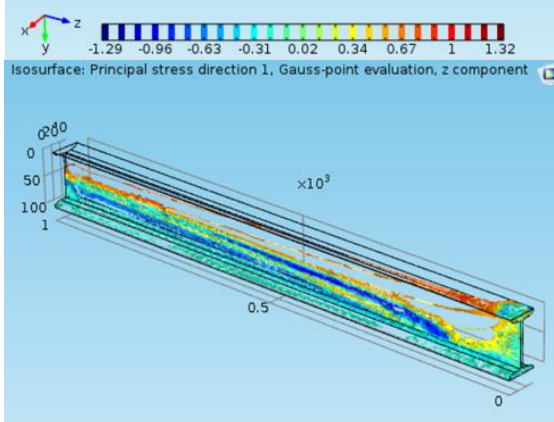

$\mathbf{F}$

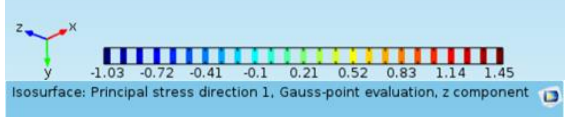

$\mathbf{H}$

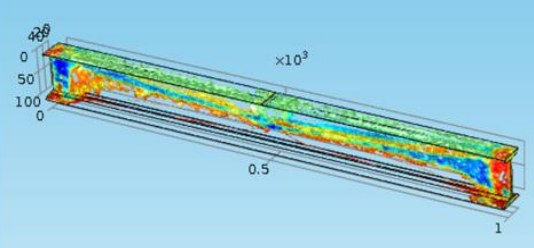

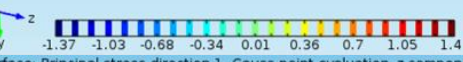
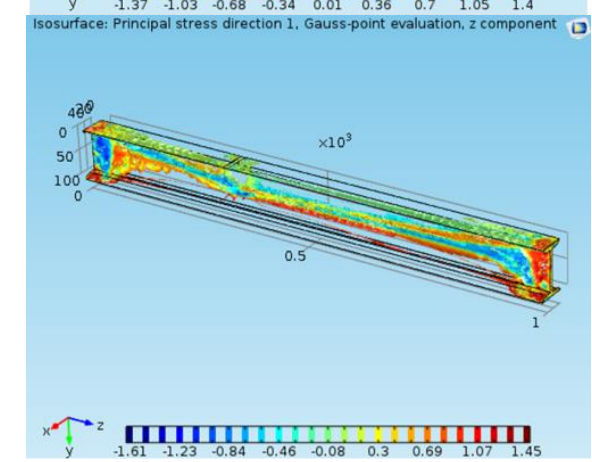

Figure 3 - Principal stress direction 1, $\mathrm{z}$ component: A - the first scheme; $\mathrm{B}$ - the second scheme; $\mathrm{C}$ - the third scheme; D - the fourth scheme; E - the fifth scheme; F - the sixth scheme; $\mathbf{G}$ - the seventh scheme; H the eighth scheme; I - the ninth scheme. 


\begin{tabular}{|c|c|c|c|c|c|c|}
\hline \multirow{4}{*}{ Impact Factor: } & ISRA (India) & $=3.117$ & SIS (USA) & $=0.912$ & ICV (Poland) & $=6.630$ \\
\hline & ISI (Dubai, UAE & $=0.829$ & РИНЦ (Russia & $=0.156$ & PIF (India) & $=1.940$ \\
\hline & GIF (Australia) & $=0.564$ & ESJI (KZ) & $=8.716$ & IBI (India) & $=4.260$ \\
\hline & JIF & $=1.500$ & SJIF (Morocco & $=5.667$ & OAJI (USA) & $=0.350$ \\
\hline
\end{tabular}

A

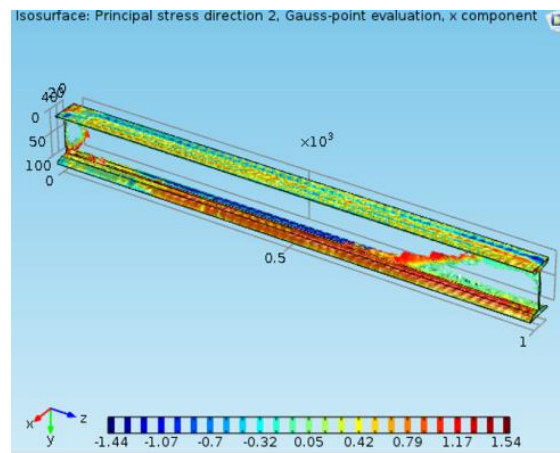

C

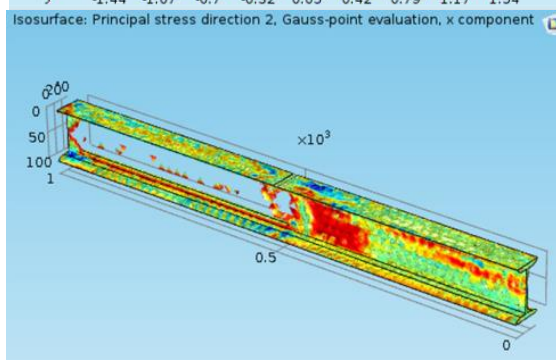

$\mathbf{E}$

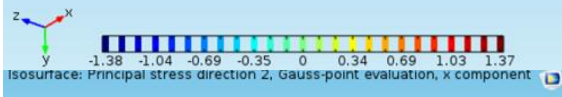

G

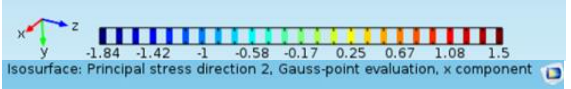

I

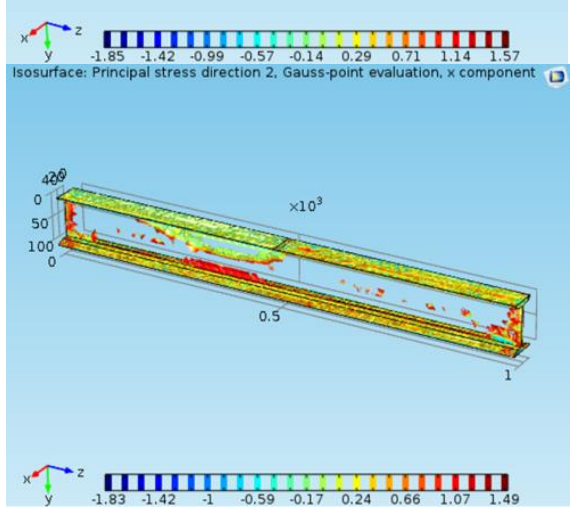

B

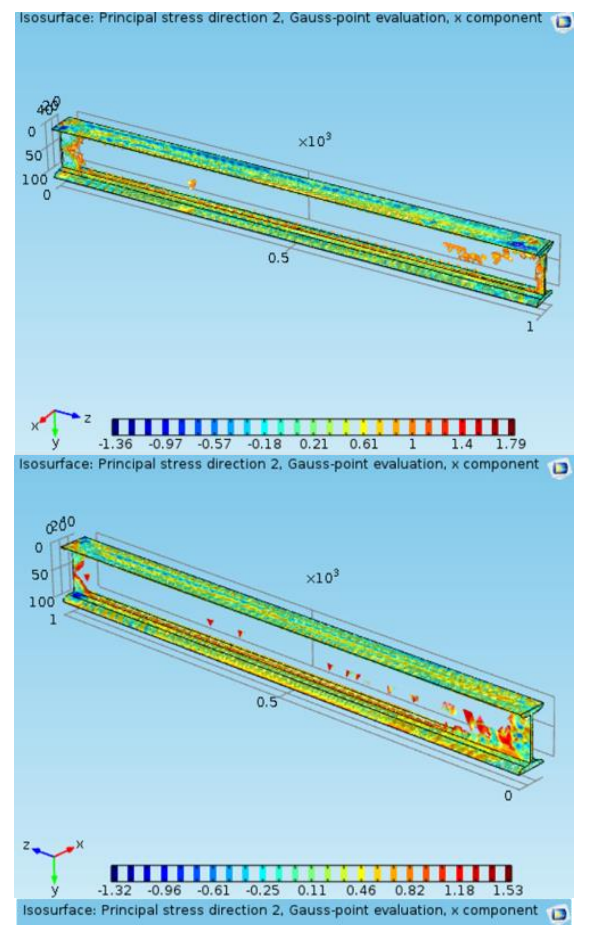

$\mathbf{F}$

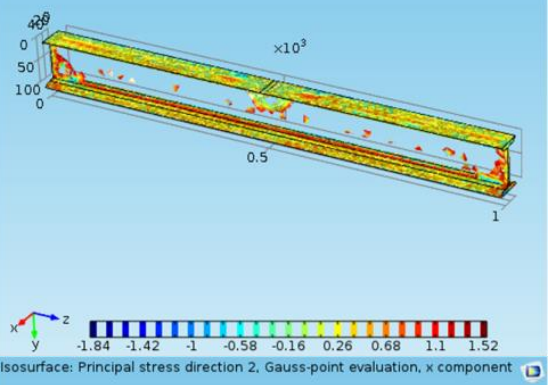

$\mathbf{H}$

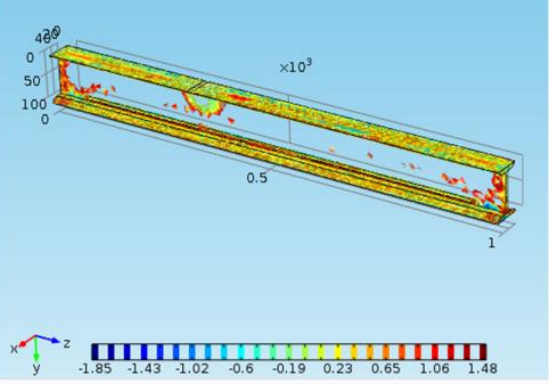

Figure 4 - Principal stress direction 2, $\mathrm{x}$ component: $\mathrm{A}$ - the first scheme; $\mathrm{B}$ - the second scheme; $\mathrm{C}$ - the third scheme; D - the fourth scheme; E - the fifth scheme; F - the sixth scheme; $\mathbf{G}$ - the seventh scheme; H the eighth scheme; I - the ninth scheme. 


\begin{tabular}{|c|c|c|c|c|c|c|}
\hline \multirow{4}{*}{ Impact Factor: } & ISRA (India) & $=3.117$ & SIS (USA) & $=0.912$ & ICV (Poland) & $=6.630$ \\
\hline & ISI (Dubai, UAE & $=0.829$ & РИНЦ (Russia & $=0.156$ & PIF (India) & $=1.940$ \\
\hline & GIF (Australia) & $=0.564$ & ESJI (KZ) & $=8.716$ & IBI (India) & $=4.260$ \\
\hline & JIF & $=1.500$ & SJIF (Morocco & $=5.667$ & OAJI (USA) & $=0.350$ \\
\hline
\end{tabular}

A

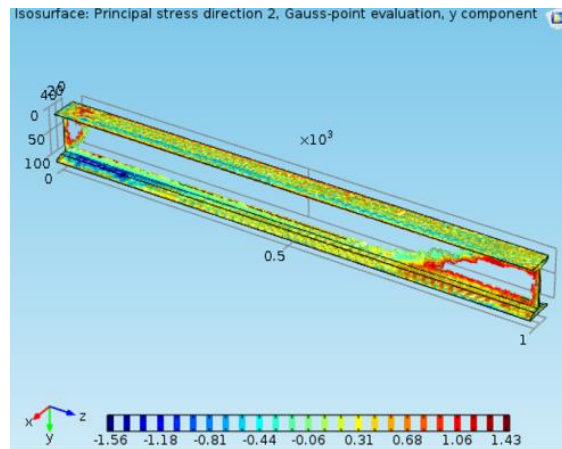

C

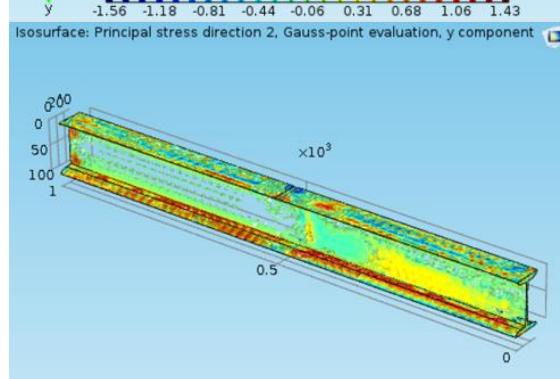

$\mathbf{E}$

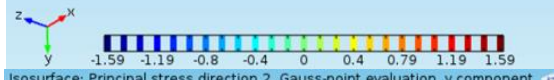

G
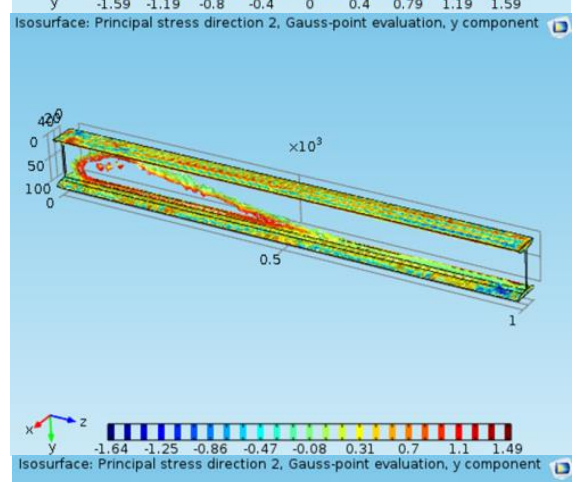

I
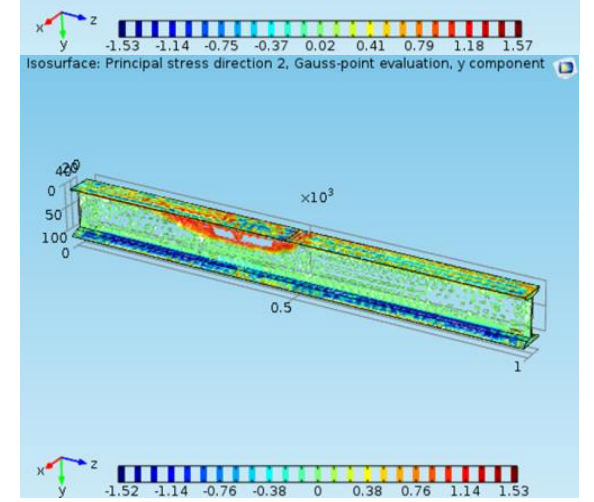

B

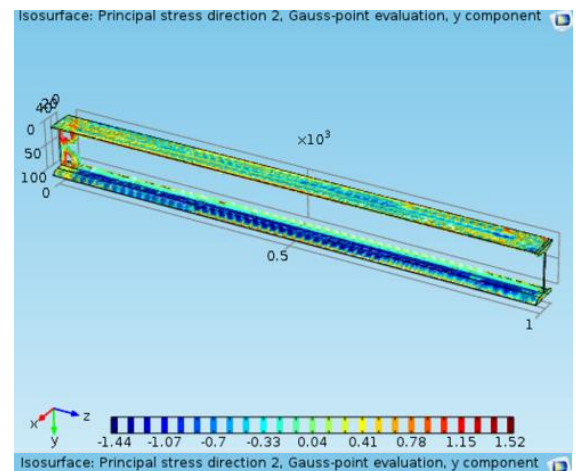

D

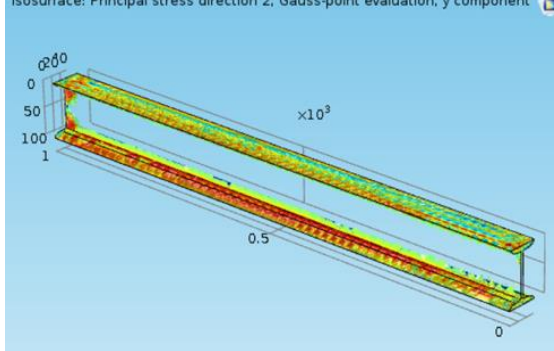

F

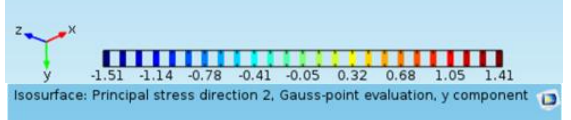

F

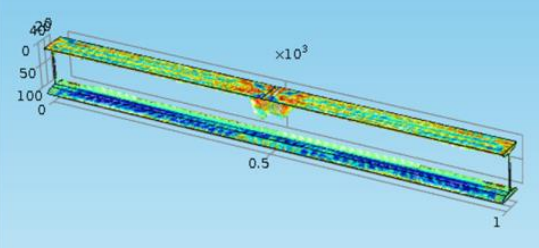

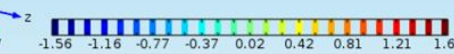
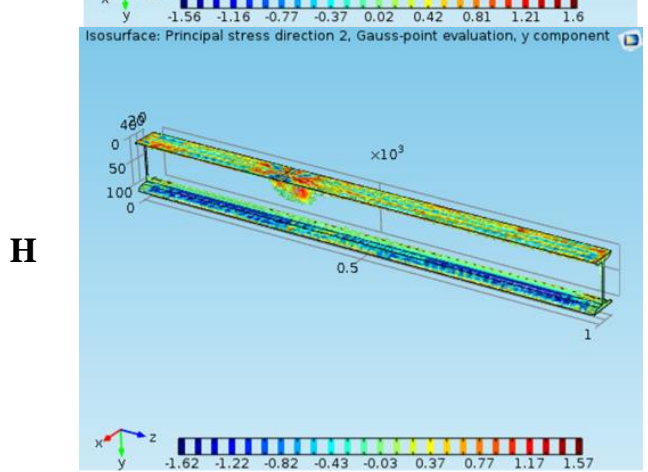

Figure 5 - Principal stress direction 2 , $\mathbf{y}$ component: A - the first scheme; $\mathbf{B}$ - the second scheme; $\mathbf{C}$ - the third scheme; D - the fourth scheme; E - the fifth scheme; F - the sixth scheme; G - the seventh scheme; H the eighth scheme; I - the ninth scheme. 


\begin{tabular}{|c|c|c|c|c|c|c|}
\hline \multirow{4}{*}{ Impact Factor: } & ISRA (India) & $=3.117$ & SIS (USA) & $=0.912$ & ICV (Poland) & $=6.630$ \\
\hline & ISI (Dubai, UAE & $=0.829$ & РИНЦ (Russia & $=0.156$ & PIF (India) & $=1.940$ \\
\hline & GIF (Australia) & $=0.564$ & ESJI (KZ) & $=8.716$ & IBI (India) & $=4.260$ \\
\hline & JIF & $=1.500$ & SJIF (Morocco & $=5.667$ & OAJI (USA) & $=0.350$ \\
\hline
\end{tabular}

A

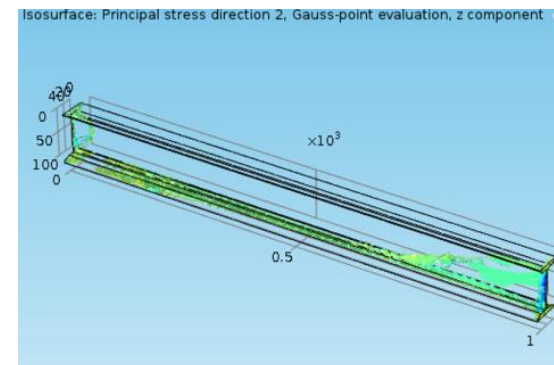

C

E

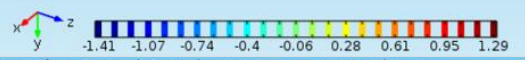
Isosurface: Principal stress direction 2, Gauss-point evaluation, $z$ component

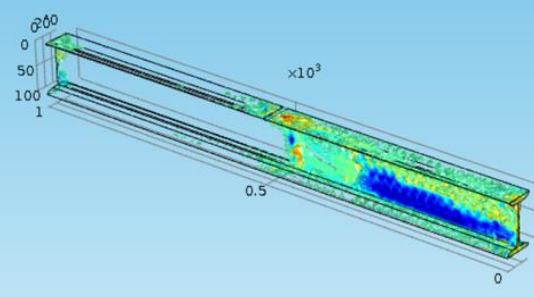

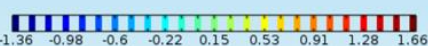

G
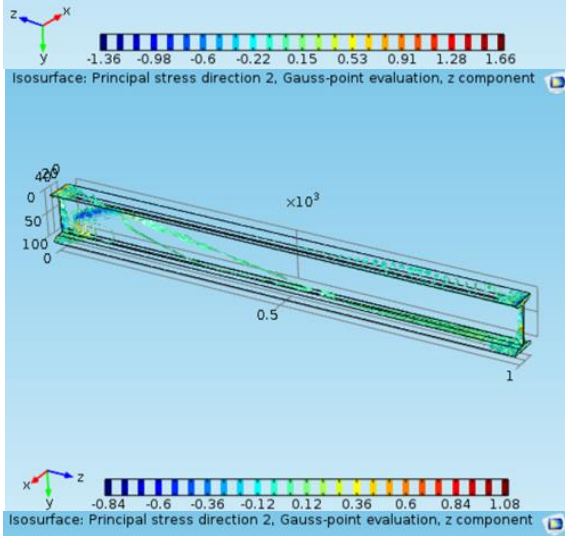

I

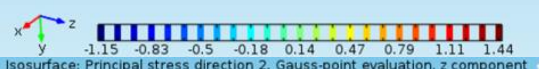

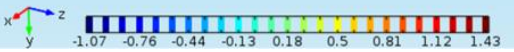

B

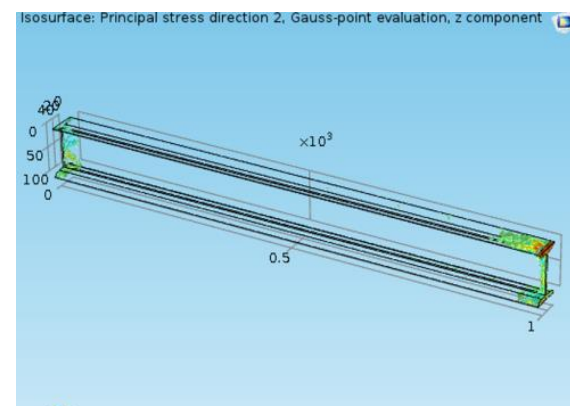

D
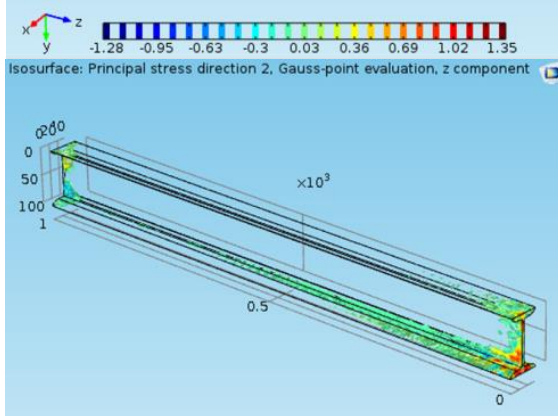

F
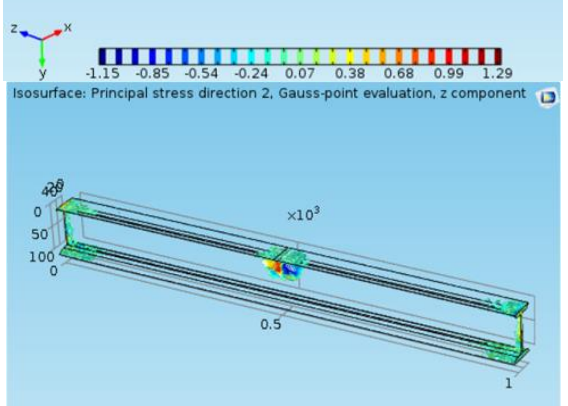

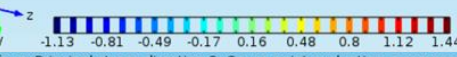
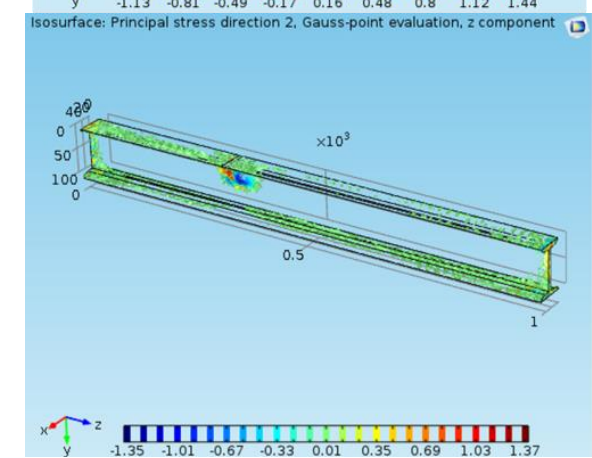

Figure 6 - Principal stress direction 2, z component: A - the first scheme; $B$ - the second scheme; $C$ - the third scheme; D - the fourth scheme; E - the fifth scheme; F - the sixth scheme; G - the seventh scheme; H the eighth scheme; I - the ninth scheme. 


\begin{tabular}{|c|c|c|c|c|c|c|}
\hline \multirow{4}{*}{ Impact Factor: } & ISRA (India) & $=3.117$ & SIS (USA) & $=0.912$ & ICV (Poland) & $=6.630$ \\
\hline & ISI (Dubai, UAE & $=0.829$ & РИНЦ (Russia & $=0.156$ & PIF (India) & $=1.940$ \\
\hline & GIF (Australia) & $=0.564$ & ESJI (KZ) & $=8.716$ & IBI (India) & $=4.260$ \\
\hline & JIF & $=1.500$ & SJIF (Morocco & $=5.667$ & OAJI (USA) & $=0.350$ \\
\hline
\end{tabular}

A

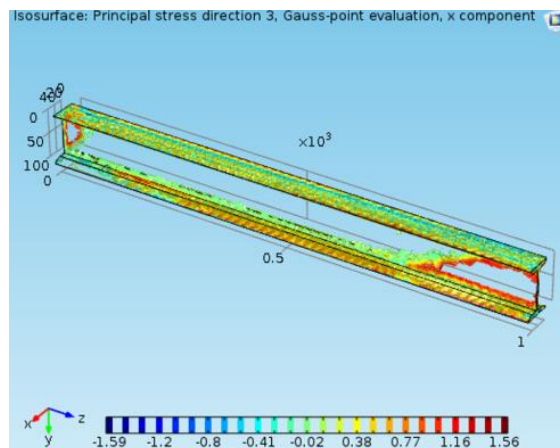

C sosurface: Principal stress difrection 3, Gussepont evaluation, x component

E
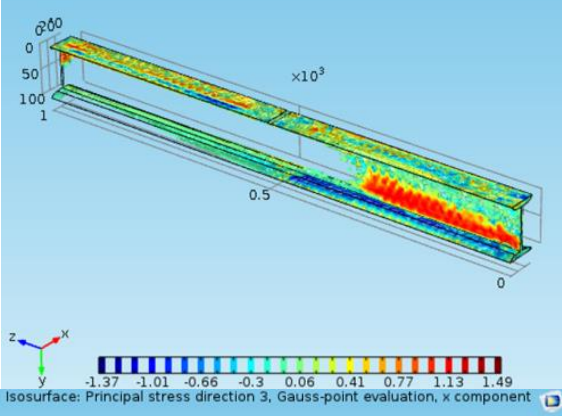

G

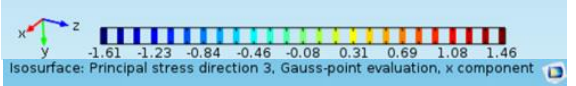

I
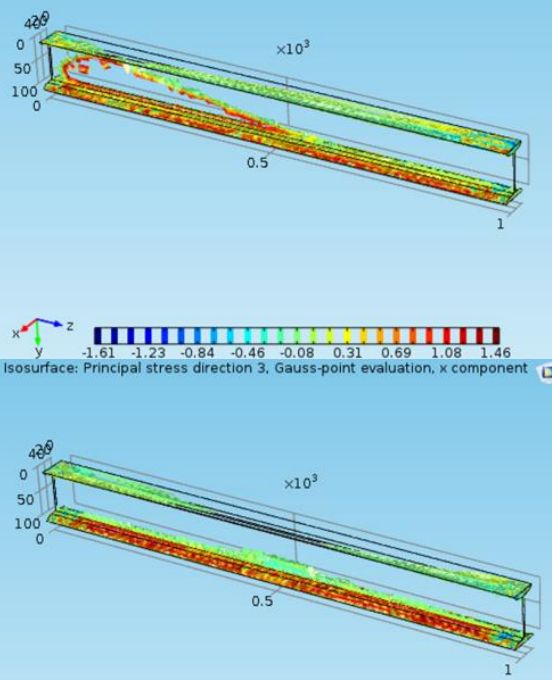

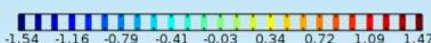
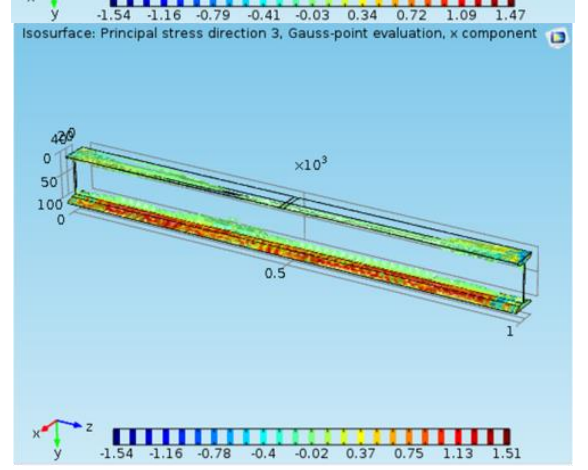

B

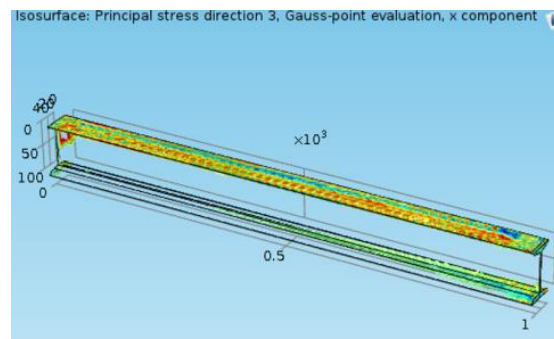

D
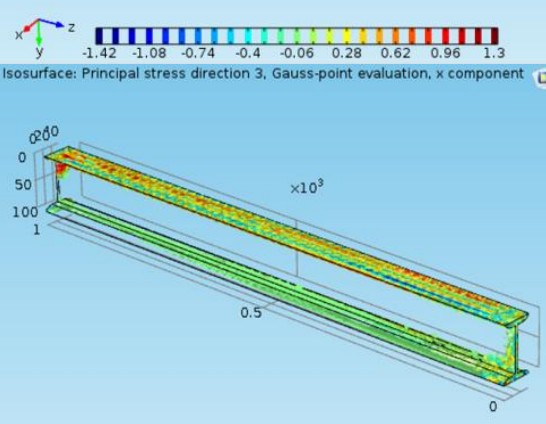

F

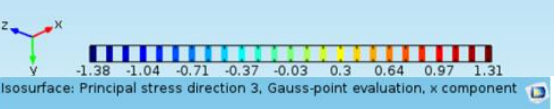

H
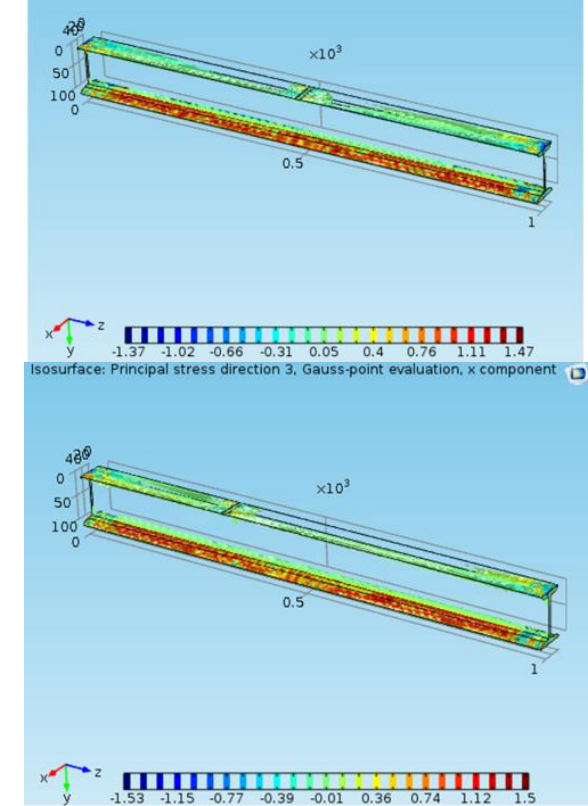

Figure 7 - Principal stress direction $3, \mathrm{x}$ component: $\mathrm{A}$ - the first scheme; $\mathrm{B}$ - the second scheme; $\mathrm{C}$ - the third scheme; D - the fourth scheme; E - the fifth scheme; F - the sixth scheme; G - the seventh scheme; H the eighth scheme; I - the ninth scheme. 


\begin{tabular}{|c|c|c|c|c|c|c|}
\hline \multirow{4}{*}{ Impact Factor: } & ISRA (India) & $=3.117$ & SIS (USA) & $=0.912$ & ICV (Poland) & $=6.630$ \\
\hline & ISI (Dubai, UAE & $=0.829$ & РИНЦ (Russia & $=0.156$ & PIF (India) & $=1.940$ \\
\hline & GIF (Australia) & $=0.564$ & ESJI (KZ) & $=8.716$ & IBI (India) & $=4.260$ \\
\hline & JIF & $=1.500$ & SJIF (Morocco & $=5.667$ & OAJI (USA) & $=0.350$ \\
\hline
\end{tabular}

A

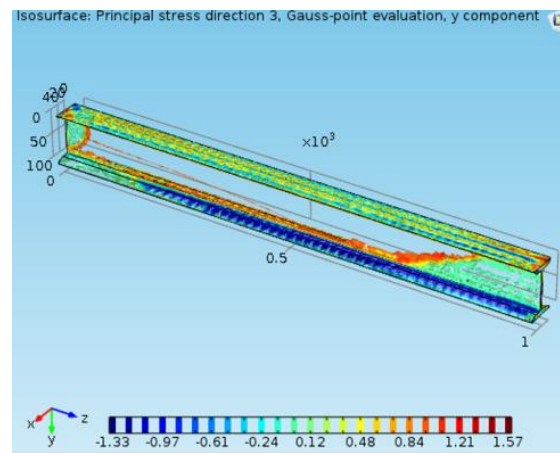

C

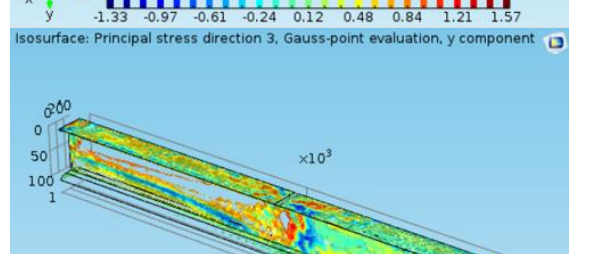

$\mathbf{E}$

G

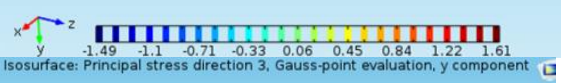

I

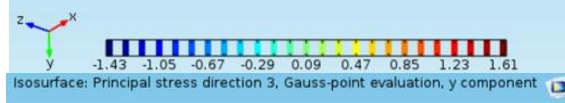

$\mathbf{E}$
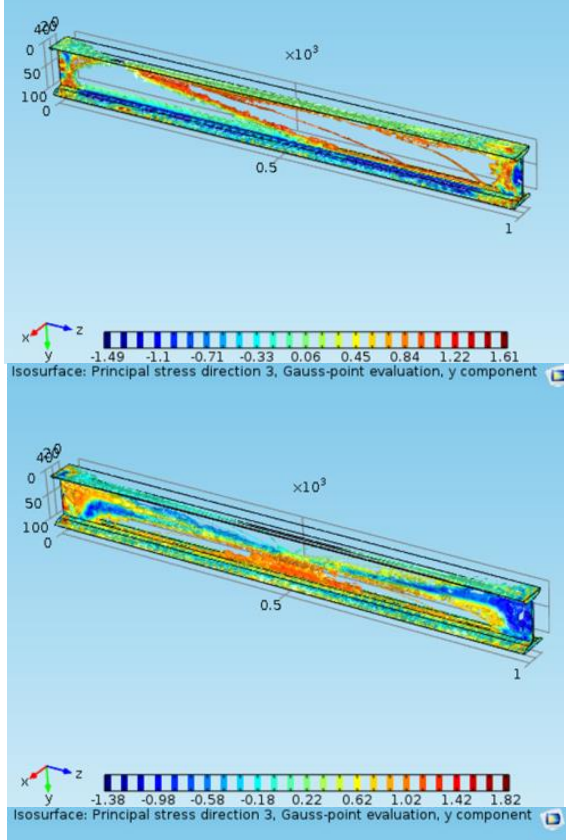

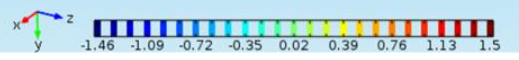

B

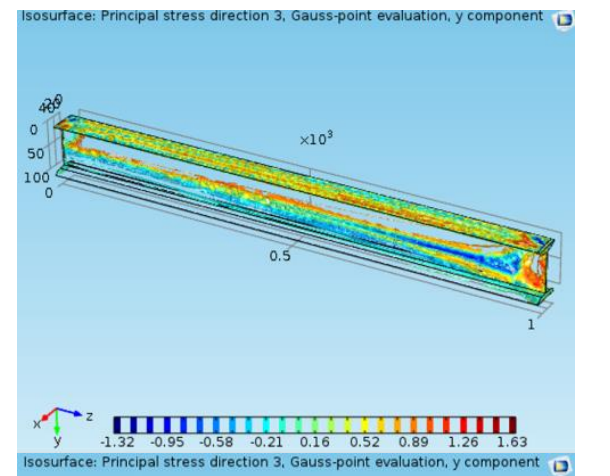

D

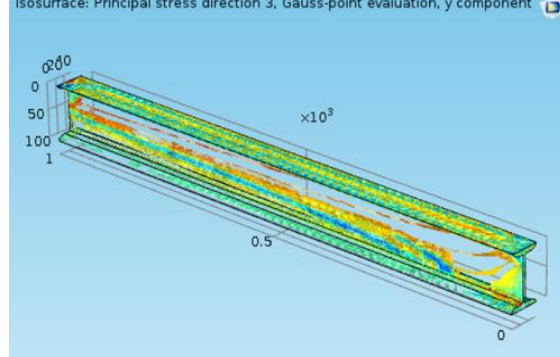

$\mathbf{F}$

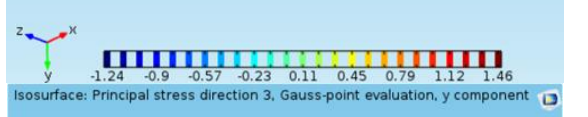

H
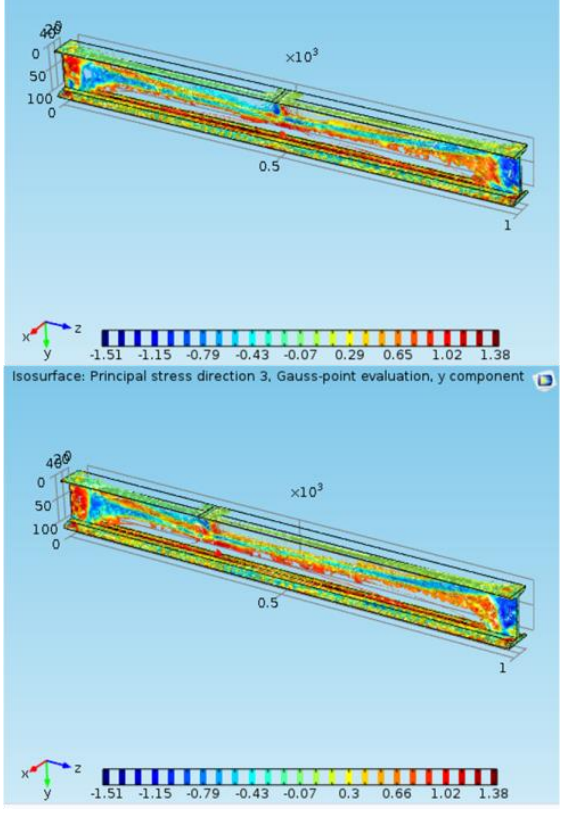

Figure 8 - Principal stress direction 3, y component: A - the first scheme; $B$ - the second scheme; $C$ - the third scheme; D - the fourth scheme; E - the fifth scheme; F - the sixth scheme; G - the seventh scheme; H the eighth scheme; I - the ninth scheme. 


\begin{tabular}{|c|c|c|c|c|c|c|}
\hline \multirow{4}{*}{ Impact Factor: } & ISRA (India) & $=3.117$ & SIS (USA) & $=0.912$ & ICV (Poland) & $=6.630$ \\
\hline & ISI (Dubai, UAE & $=0.829$ & РИНЦ (Russia) & $=0.156$ & PIF (India) & $=1.940$ \\
\hline & GIF (Australia) & $=0.564$ & ESJI (KZ) & $=8.716$ & IBI (India) & $=4.260$ \\
\hline & JIF & $=1.500$ & SJIF (Morocco) & $=5.667$ & OAJI (USA) & $=0.350$ \\
\hline
\end{tabular}

A

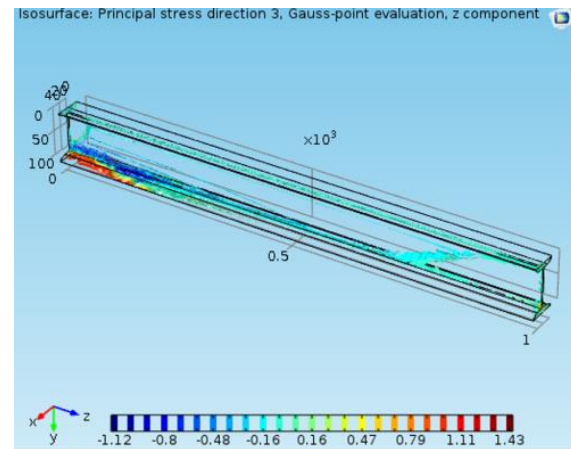

C

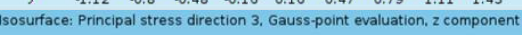

$\mathbf{E}$

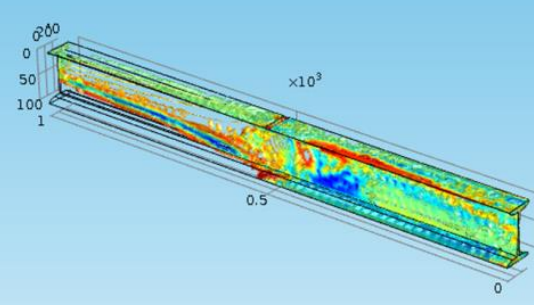

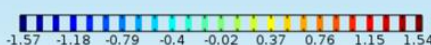

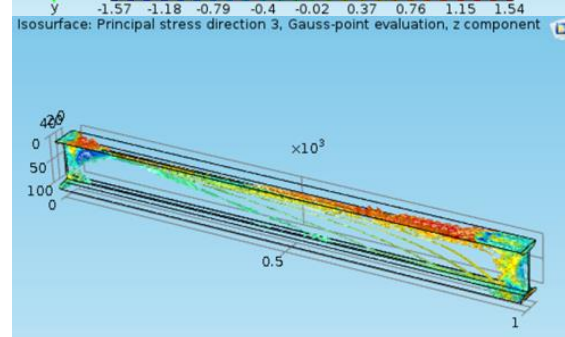

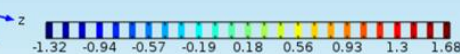
Isosurface: Principal stress direction 3, Gauss-point evaluation, 2 component

G

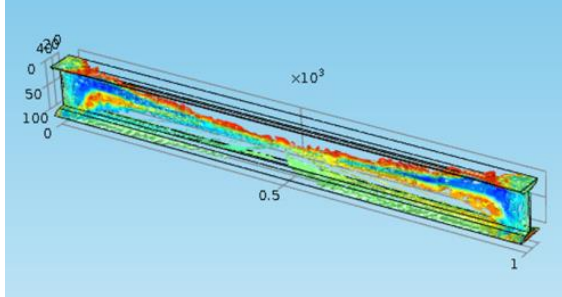

I

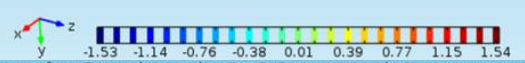

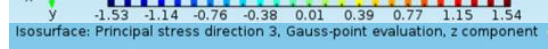

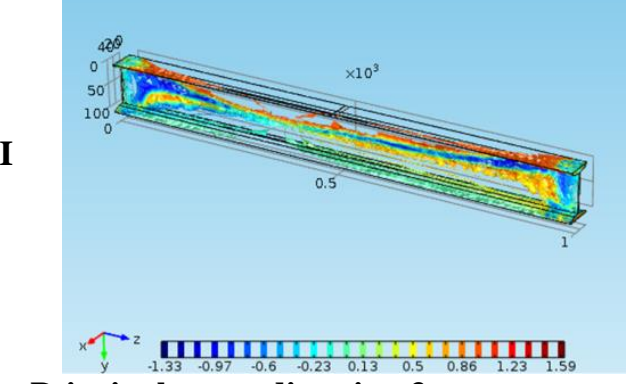

Figure 9 - Principal stress direction 3, z component: A - the first scheme; $B$ - the second scheme; $C$ - the third scheme; D - the fourth scheme; E - the fifth scheme; F - the sixth scheme; G - the seventh scheme; H the eighth scheme; I - the ninth scheme. 


\begin{tabular}{|c|c|c|c|c|c|c|}
\hline \multirow{4}{*}{ Impact Factor: } & ISRA (India) & $=3.117$ & SIS (USA) & $=0.912$ & ICV (Poland) & $=6.630$ \\
\hline & ISI (Dubai, UAE & $=0.829$ & РИНЦ (Russia) & $=0.156$ & PIF (India) & $=1.940$ \\
\hline & GIF (Australia) & $=0.564$ & ESJI (KZ) & $=8.716$ & IBI (India) & $=4.260$ \\
\hline & JIF & $=1.500$ & SJIF (Morocco) & $=5.667$ & OAJI (USA) & $=0.350$ \\
\hline
\end{tabular}

A

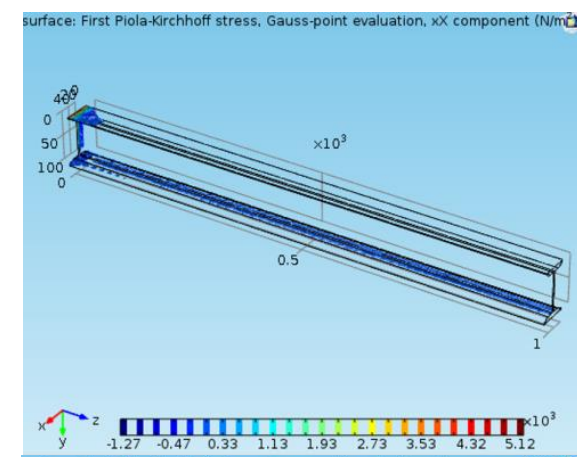

C
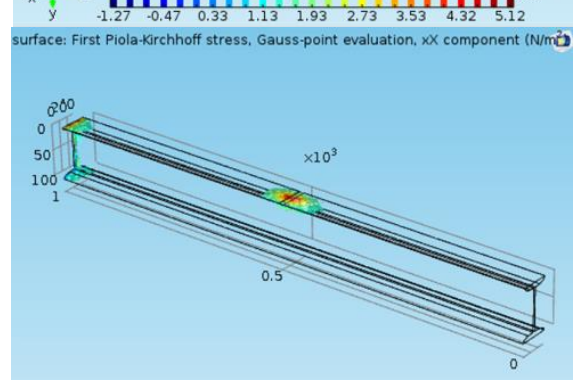

$\mathbf{E}$

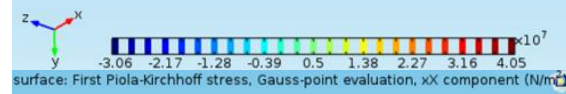

G
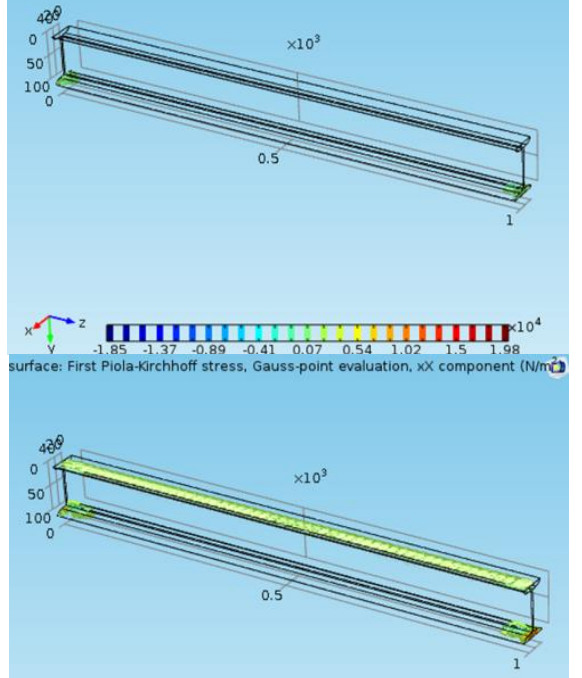

I

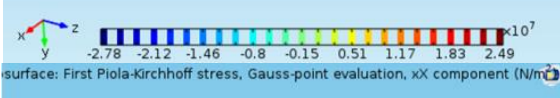

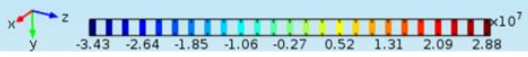

B

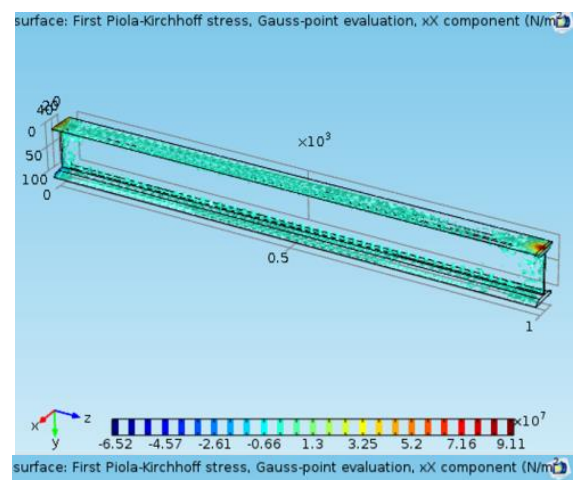

D

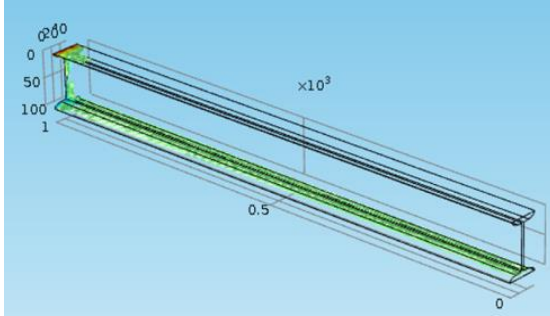

$\mathbf{F}$

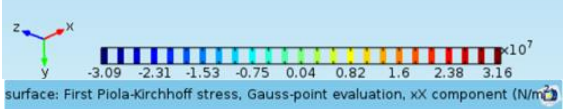

$\mathbf{H}$
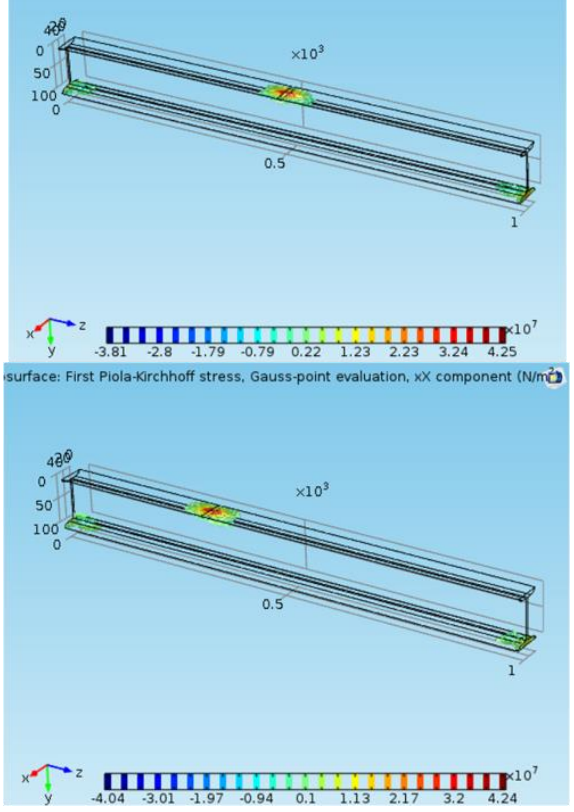

Figure 10 - First Piola-Kirchhoff stress, $\mathrm{XX}$ component: A - the first scheme; $\mathrm{B}$ - the second scheme; $\mathrm{C}$ - the third scheme; D - the fourth scheme; E - the fifth scheme; F - the sixth scheme; G - the seventh scheme; H the eighth scheme; I - the ninth scheme. 


\begin{tabular}{|c|c|c|c|c|c|c|}
\hline \multirow{4}{*}{ Impact Factor: } & ISRA (India) & $=3.117$ & SIS (USA) & $=0.912$ & ICV (Poland) & $=6.630$ \\
\hline & ISI (Dubai, UAE & $=0.829$ & РИНЦ (Russia & $=0.156$ & PIF (India) & $=1.940$ \\
\hline & GIF (Australia) & $=0.564$ & ESJI (KZ) & $=8.716$ & IBI (India) & $=4.260$ \\
\hline & JIF & $=1.500$ & SJIF (Morocco & $=5.667$ & OAJI (USA) & $=0.350$ \\
\hline
\end{tabular}

A

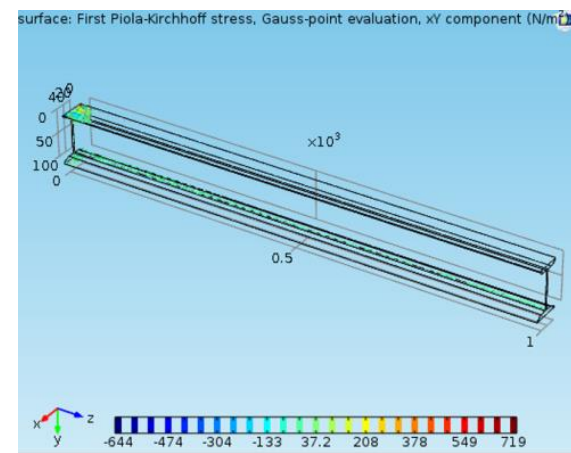

C

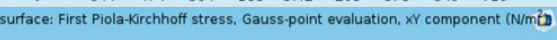

$\mathbf{E}$

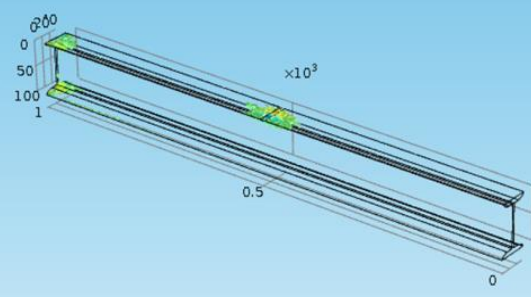

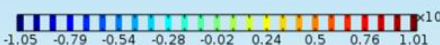
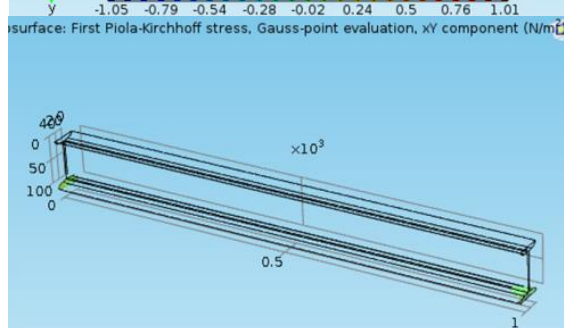

G
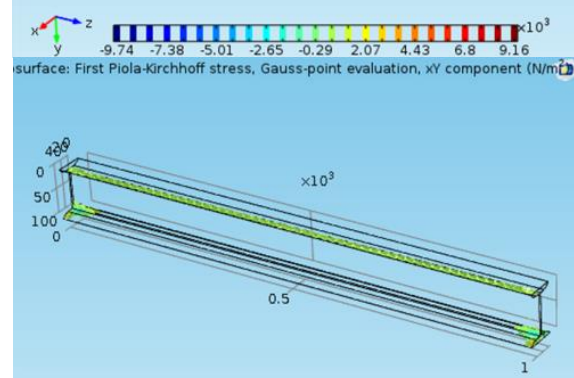

I

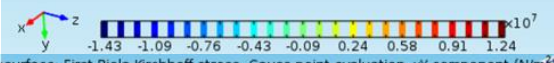

B

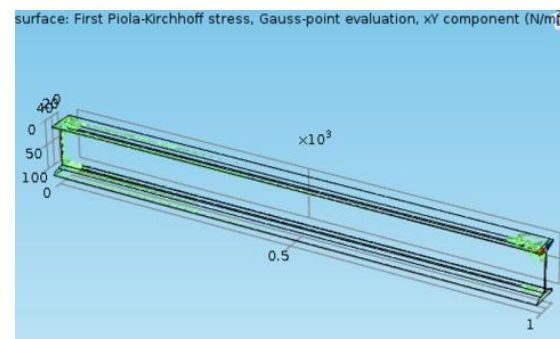

D
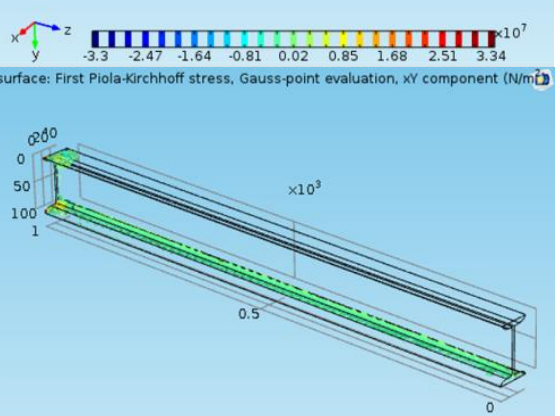

F

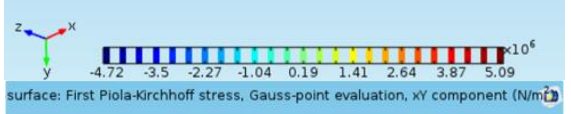

H
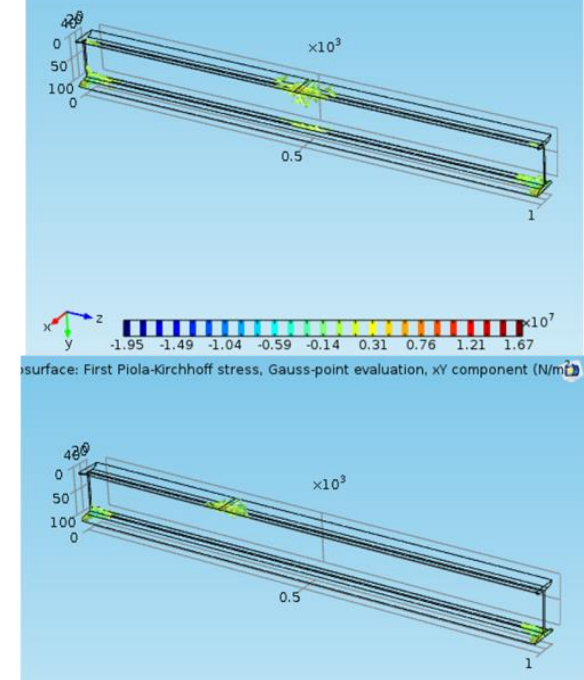

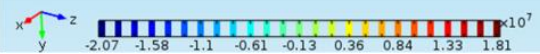

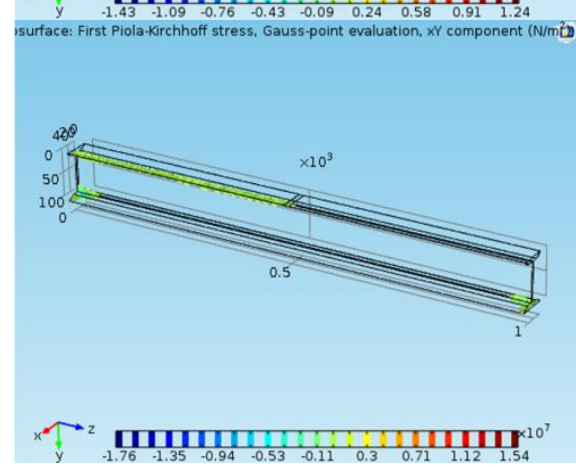

Figure 11 - First Piola-Kirchhoff stress, $\mathrm{xY}$ component: A - the first scheme; $\mathrm{B}$ - the second scheme; $\mathrm{C}$ - the third scheme; D - the fourth scheme; E - the fifth scheme; F - the sixth scheme; G - the seventh scheme; H the eighth scheme; I - the ninth scheme. 


\begin{tabular}{|c|c|c|c|c|c|c|}
\hline \multirow{4}{*}{ Impact Factor: } & ISRA (India) & $=3.117$ & SIS (USA) & $=0.912$ & ICV (Poland) & $=6.630$ \\
\hline & ISI (Dubai, UAE & $=0.829$ & РИНЦ (Russia & $=0.156$ & PIF (India) & $=1.940$ \\
\hline & GIF (Australia) & $=0.564$ & ESJI (KZ) & $=8.716$ & IBI (India) & $=4.260$ \\
\hline & JIF & $=1.500$ & SJIF (Morocco & $=5.667$ & OAJI (USA) & $=0.350$ \\
\hline
\end{tabular}

A

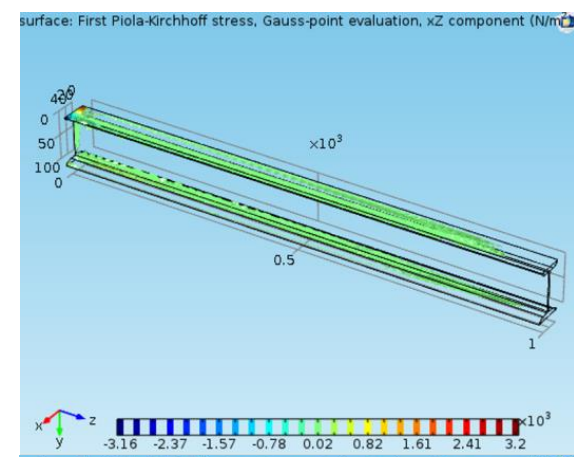

C

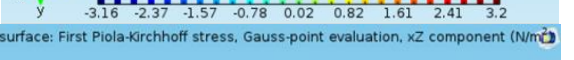

$\mathbf{E}$
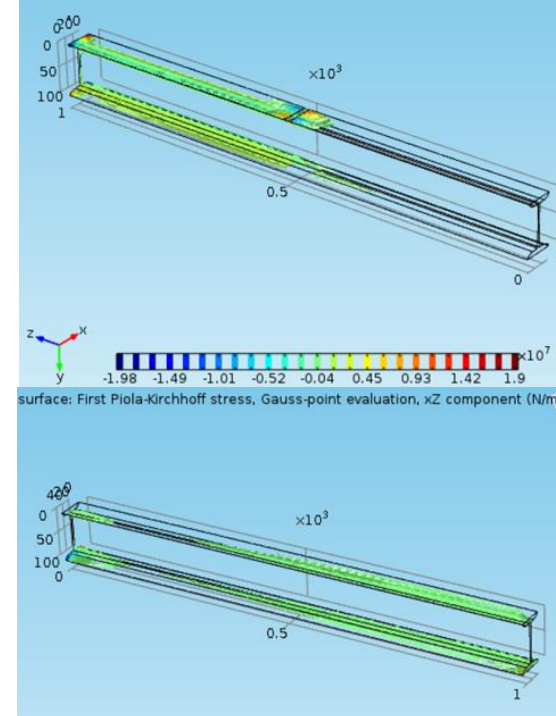

G
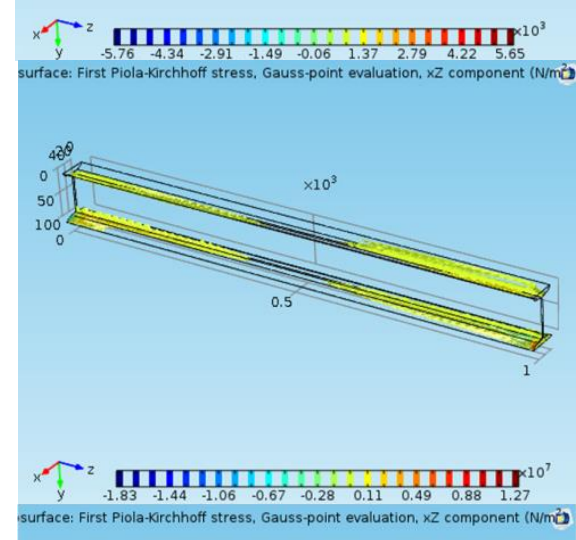

I

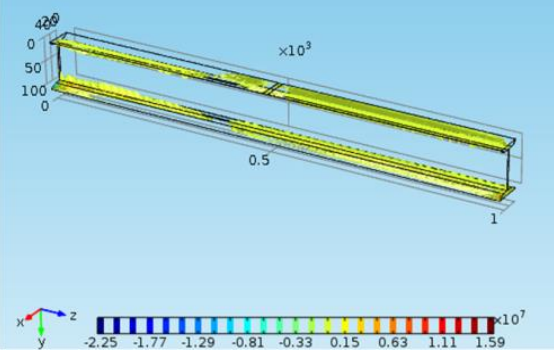

B

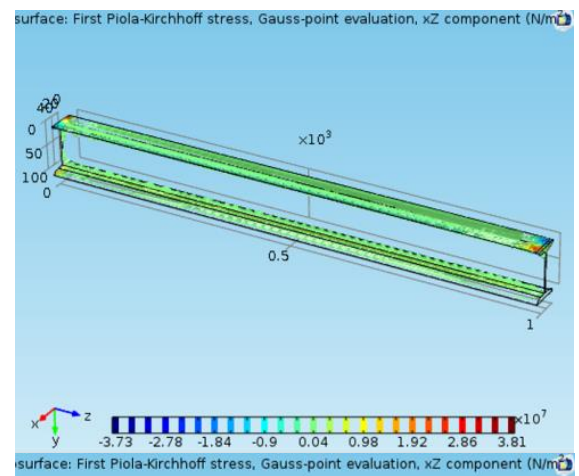

D

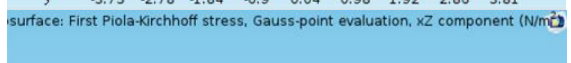

$\mathbf{F}$
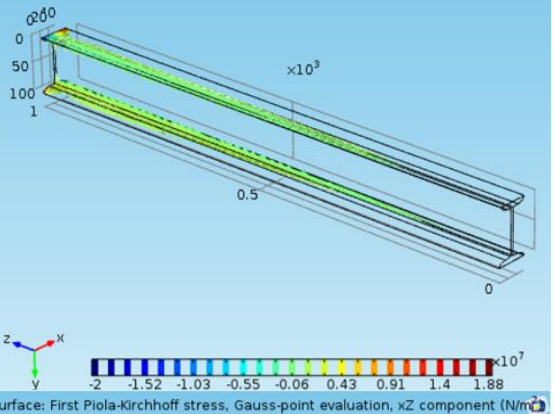

$\mathbf{H}$
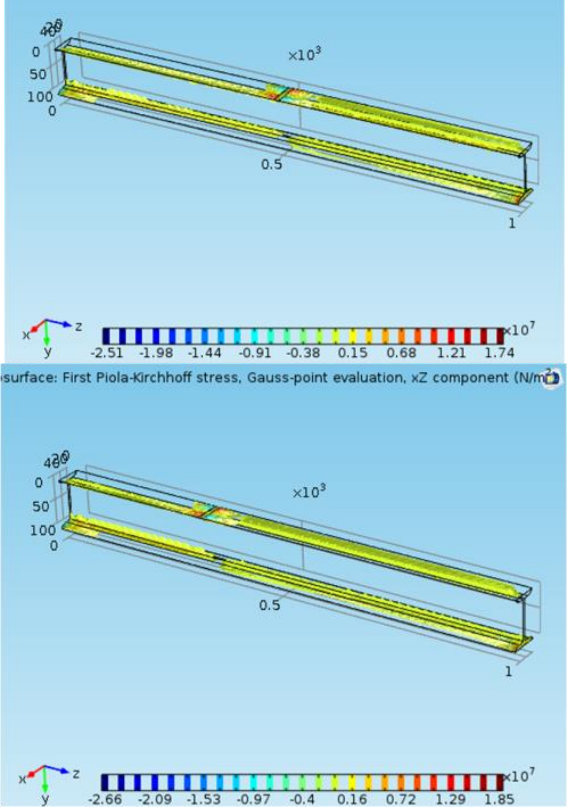

Figure 12 - First Piola-Kirchhoff stress, $x Z$ component: A - the first scheme; $B$ - the second scheme; $C$ - the third scheme; D - the fourth scheme; E - the fifth scheme; F - the sixth scheme; G - the seventh scheme; H the eighth scheme; I - the ninth scheme. 


\begin{tabular}{|c|c|c|c|c|c|c|}
\hline \multirow{4}{*}{ Impact Factor: } & ISRA (India) & $=3.117$ & SIS (USA) & $=0.912$ & ICV (Poland) & $=6.630$ \\
\hline & ISI (Dubai, UAE & $=0.829$ & РИНЦ (Russia & $=0.156$ & PIF (India) & $=1.940$ \\
\hline & GIF (Australia) & $=0.564$ & ESJI (KZ) & $=8.716$ & IBI (India) & $=4.260$ \\
\hline & JIF & $=1.500$ & SJIF (Morocco & $=5.667$ & OAJI (USA) & $=0.350$ \\
\hline
\end{tabular}

A

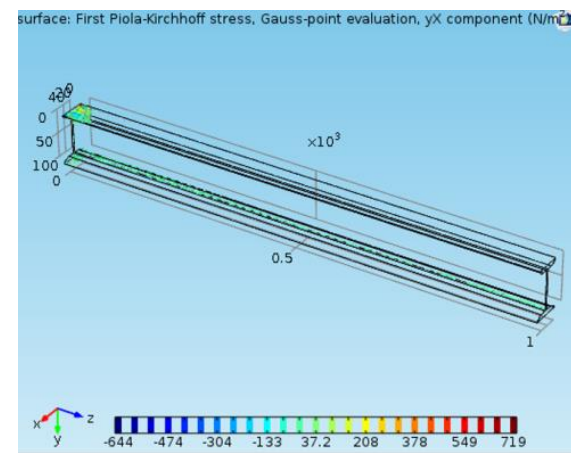

C

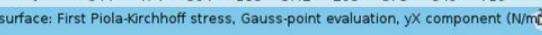

$\mathbf{E}$
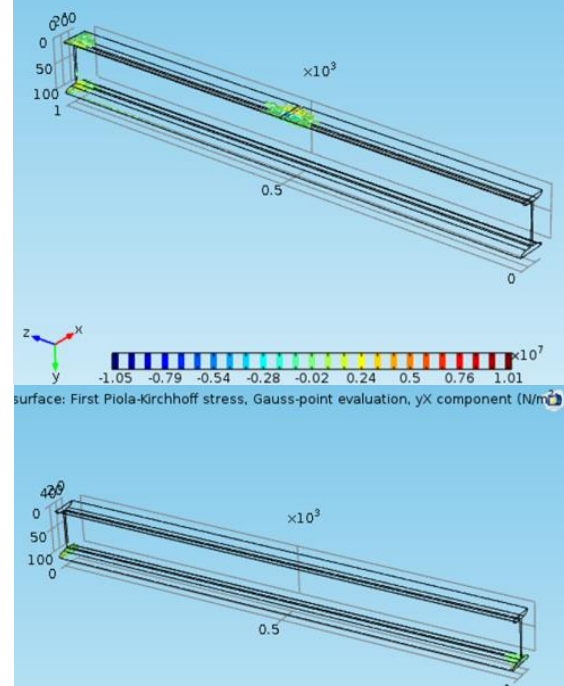

G
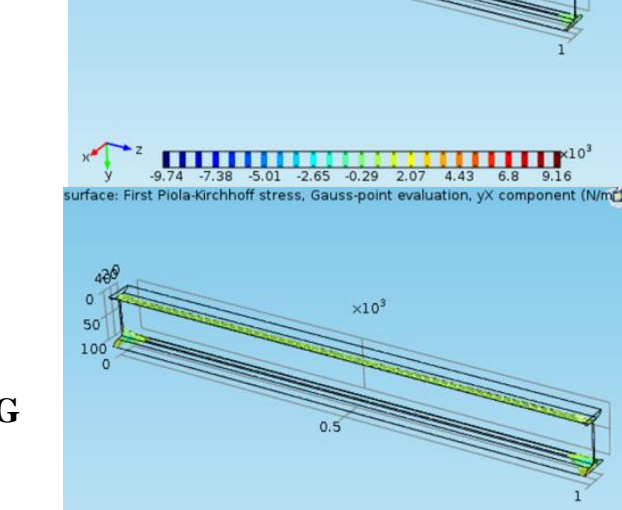

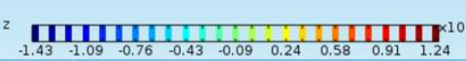

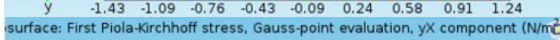

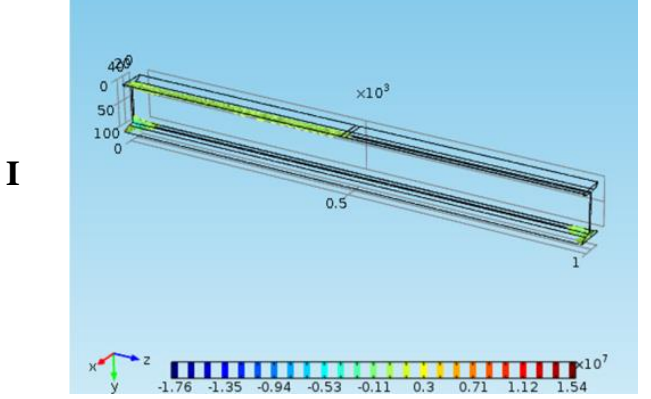

B

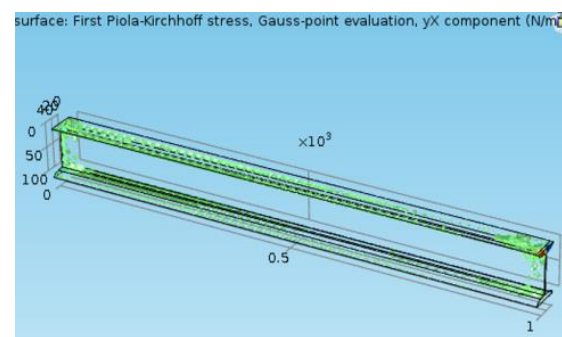

D

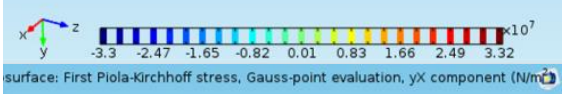

$\mathbf{F}$
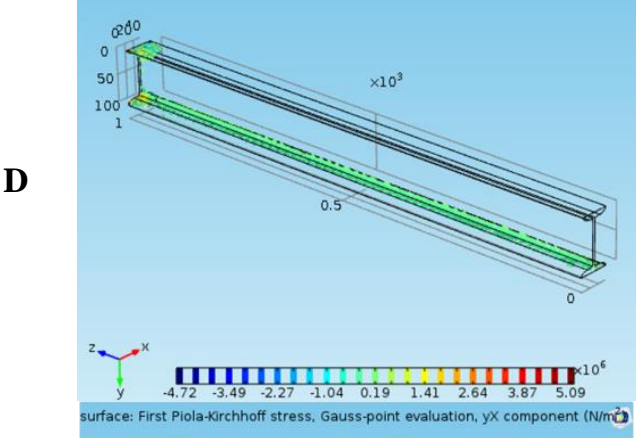

$\mathbf{H}$
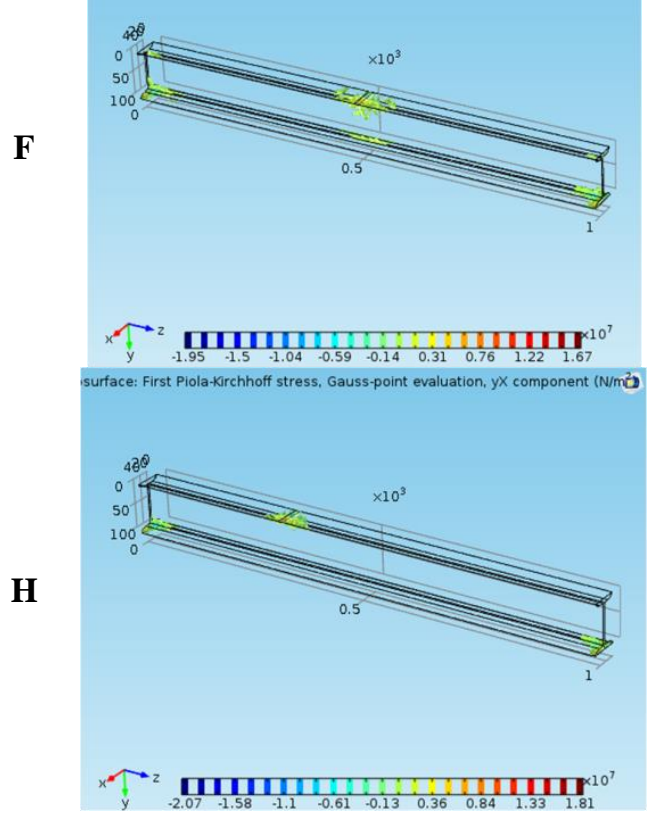

Figure 13 - First Piola-Kirchhoff stress, yX component: A - the first scheme; B - the second scheme; C - the third scheme; D - the fourth scheme; $\mathrm{E}$ - the fifth scheme; F - the sixth scheme; $\mathbf{G}$ - the seventh scheme; H the eighth scheme; I - the ninth scheme. 


\begin{tabular}{|c|c|c|c|c|c|c|}
\hline \multirow{4}{*}{ Impact Factor: } & ISRA (India) & $=3.117$ & SIS (USA) & $=0.912$ & ICV (Poland) & $=6.630$ \\
\hline & ISI (Dubai, UAE & $=0.829$ & РИНЦ (Russia & $=0.156$ & PIF (India) & $=1.940$ \\
\hline & GIF (Australia) & $=0.564$ & ESJI (KZ) & $=8.716$ & IBI (India) & $=4.260$ \\
\hline & JIF & $=1.500$ & SJIF (Morocco & $=5.667$ & OAJI (USA) & $=0.350$ \\
\hline
\end{tabular}

A

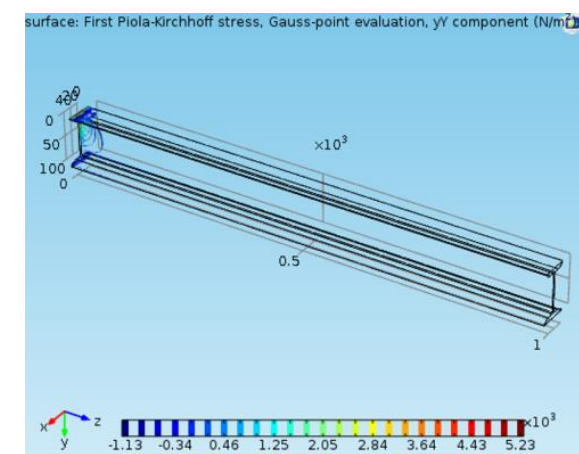

C
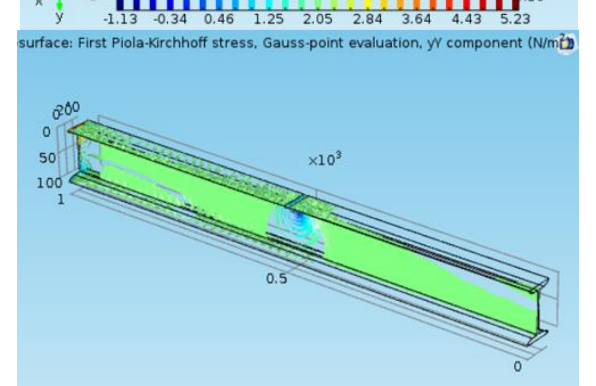

$\mathbf{E}$

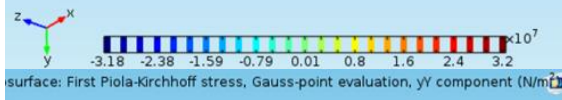

G
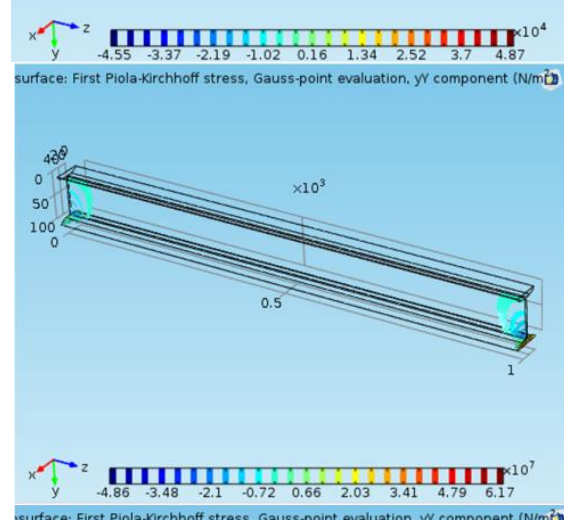

I

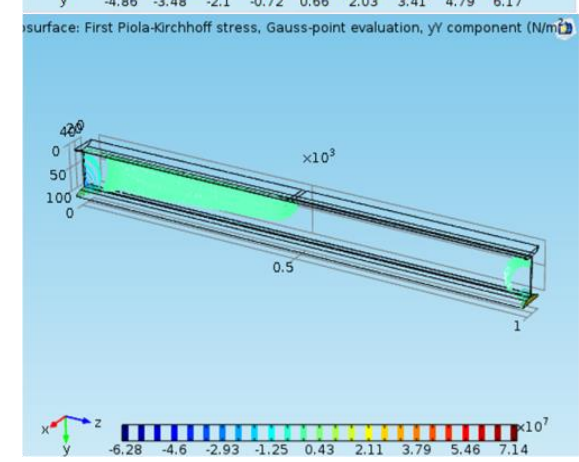

B

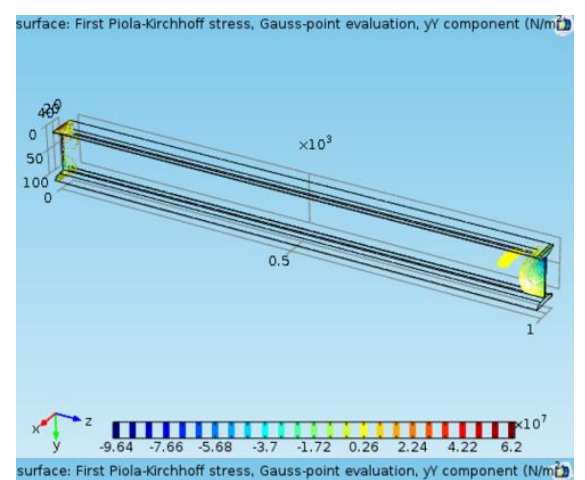

D

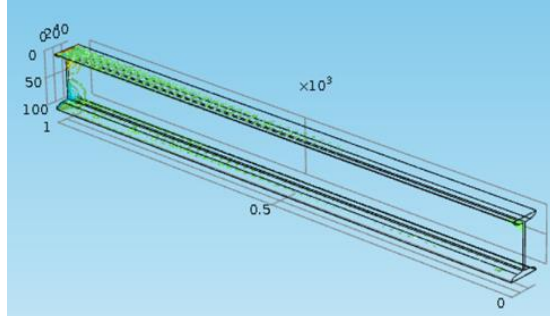

F

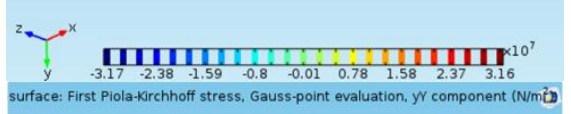

H
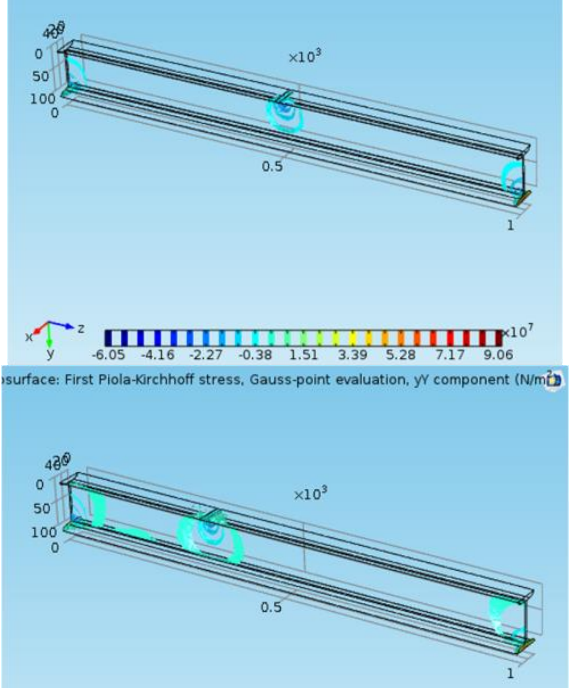

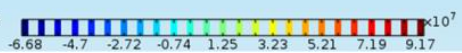

Figure 14 - First Piola-Kirchhoff stress, yY component: A - the first scheme; B - the second scheme; C - the third scheme; D - the fourth scheme; E - the fifth scheme; F - the sixth scheme; $\mathbf{G}$ - the seventh scheme; H the eighth scheme; I - the ninth scheme. 


\begin{tabular}{|c|c|c|c|c|c|c|}
\hline \multirow{4}{*}{ Impact Factor: } & ISRA (India) & $=3.117$ & SIS (USA) & $=0.912$ & ICV (Poland) & $=6.630$ \\
\hline & ISI (Dubai, UAE & $=0.829$ & РИНЦ (Russia & $=0.156$ & PIF (India) & $=1.940$ \\
\hline & GIF (Australia) & $=0.564$ & ESJI (KZ) & $=8.716$ & IBI (India) & $=4.260$ \\
\hline & JIF & $=1.500$ & SJIF (Morocco & $=5.667$ & OAJI (USA) & $=0.350$ \\
\hline
\end{tabular}

A

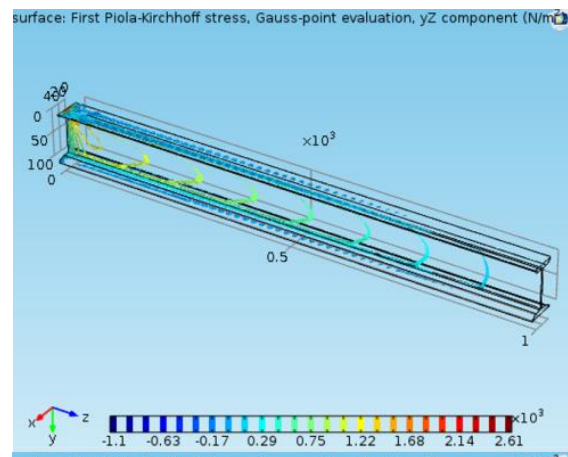

surface: First Piola-Kirchhoff stress, Gauss.point evaluation, yz component (N/ma

C

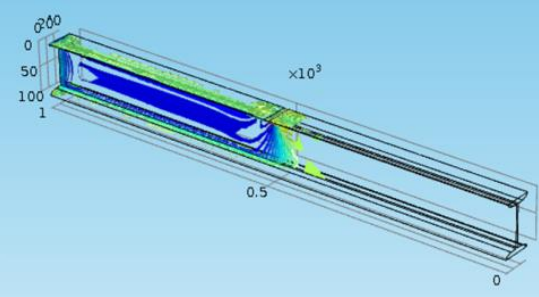

$\mathbf{E}$

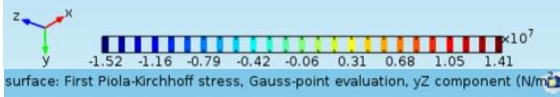

G
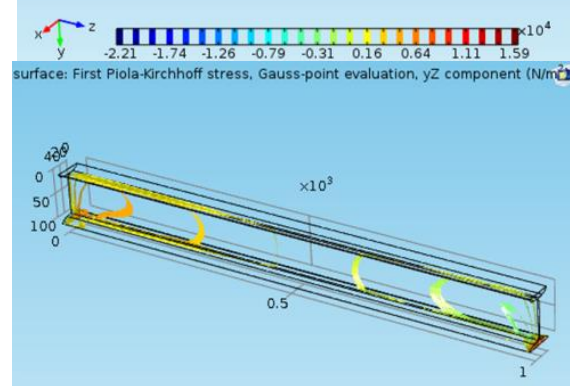

I

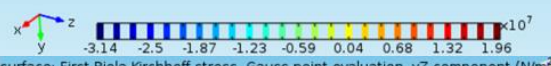

B

surface: First Piola-kirchhoff stress, Gauss-point evaluation, yz component (N/m²

D
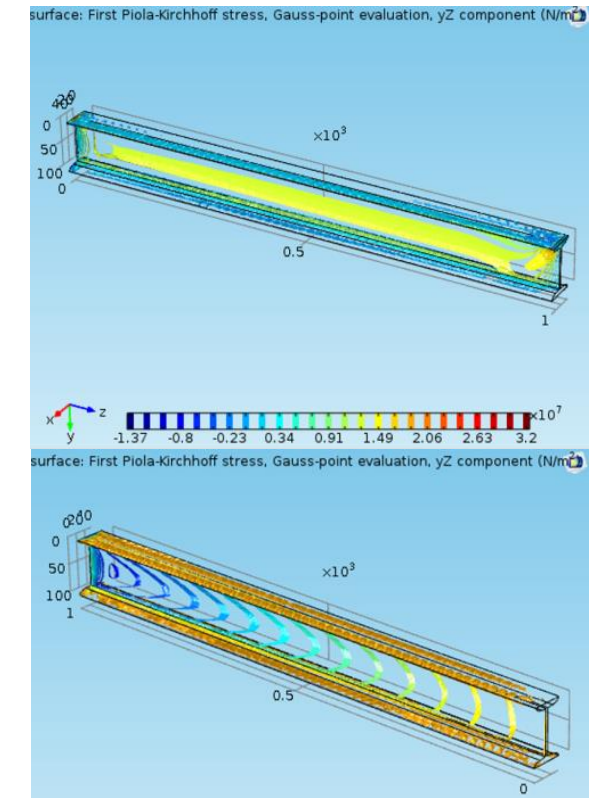

F

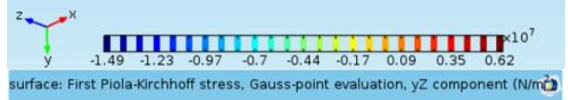

H
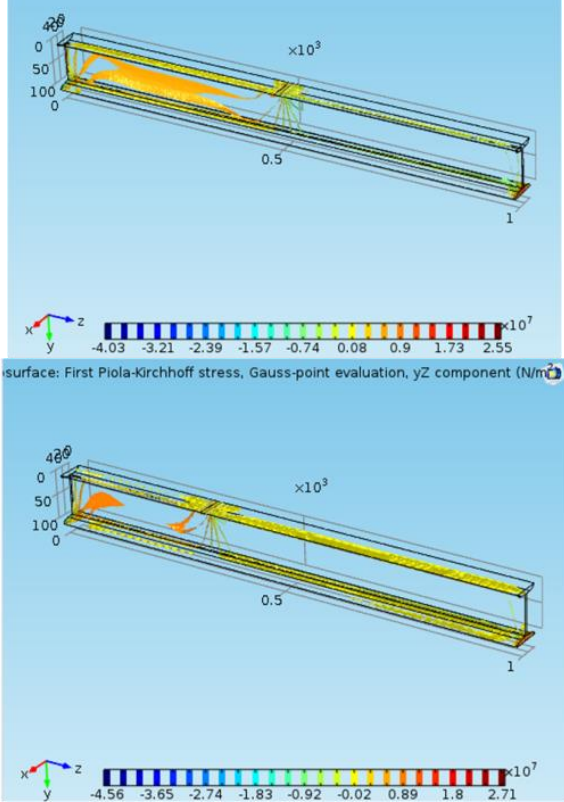

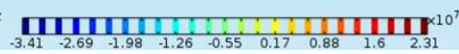

Figure 15 - First Piola-Kirchhoff stress, yZ component: A - the first scheme; B - the second scheme; $\mathrm{C}$ - the third scheme; D - the fourth scheme; E - the fifth scheme; F - the sixth scheme; G - the seventh scheme; H the eighth scheme; I - the ninth scheme. 


\begin{tabular}{|c|c|c|c|c|c|c|}
\hline \multirow{4}{*}{ Impact Factor: } & ISRA (India) & $=3.117$ & SIS (USA) & $=0.912$ & ICV (Poland) & $=6.630$ \\
\hline & ISI (Dubai, UAE & $=0.829$ & РИНЦ (Russia & $=0.156$ & PIF (India) & $=1.940$ \\
\hline & GIF (Australia) & $=0.564$ & ESJI (KZ) & $=8.716$ & IBI (India) & $=4.260$ \\
\hline & JIF & $=1.500$ & SJIF (Morocco & $=5.667$ & OAJI (USA) & $=0.350$ \\
\hline
\end{tabular}

A
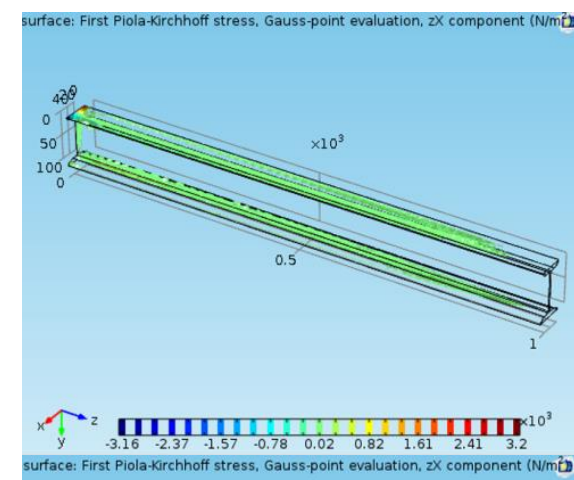

C

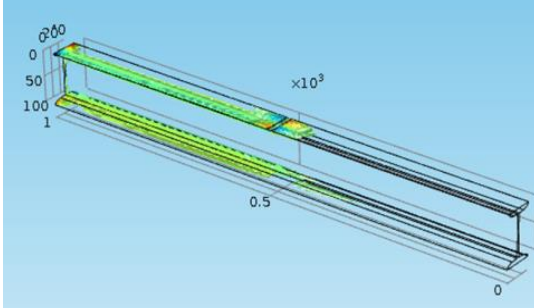

$\mathbf{E}$

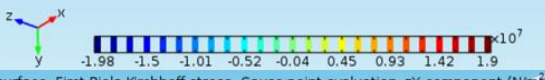

G
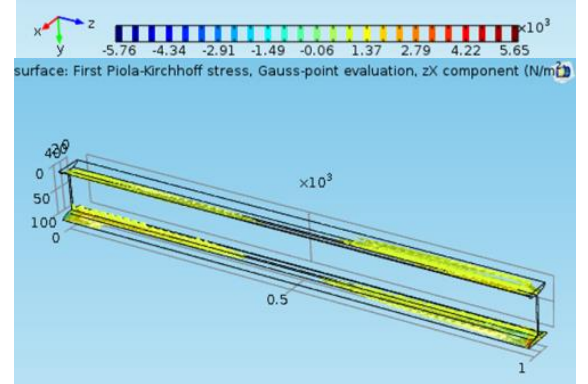

I

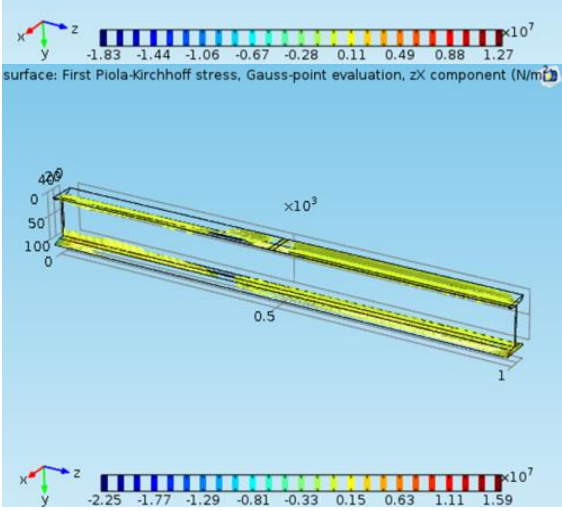

B

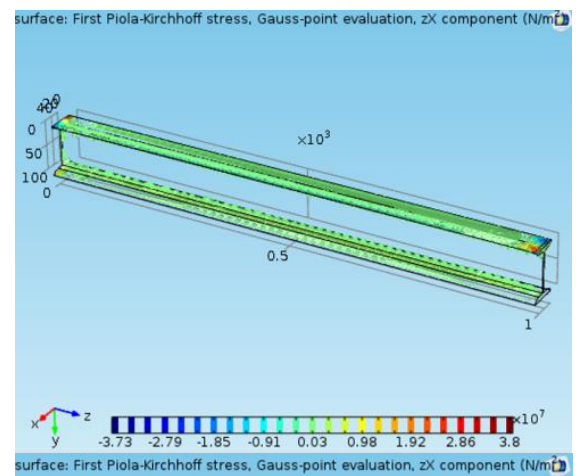

D
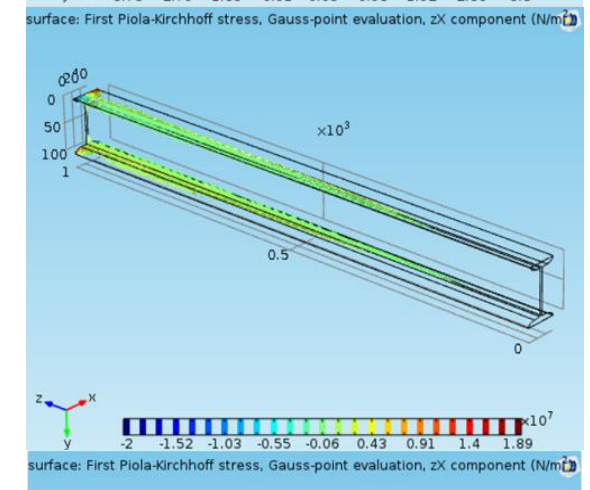

$\mathbf{F}$

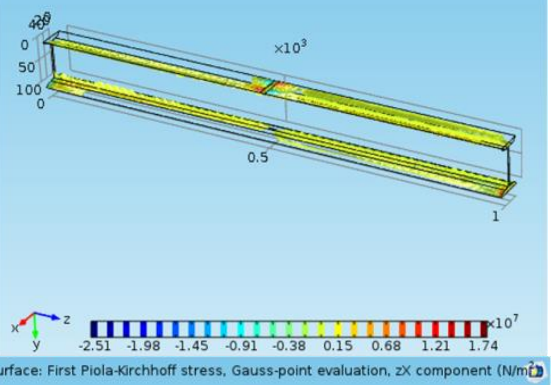

H

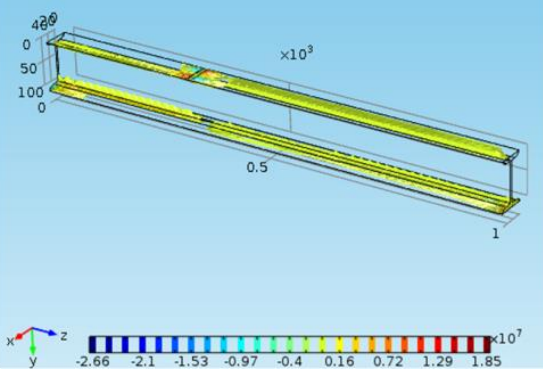

Figure 16 - First Piola-Kirchhoff stress, zX component: A - the first scheme; B - the second scheme; C - the third scheme; D - the fourth scheme; E - the fifth scheme; F - the sixth scheme; $\mathbf{G}$ - the seventh scheme; H the eighth scheme; I - the ninth scheme. 


\begin{tabular}{|c|c|c|c|c|c|c|}
\hline \multirow{4}{*}{ Impact Factor: } & ISRA (India) & $=3.117$ & SIS (USA) & $=0.912$ & ICV (Poland) & $=6.630$ \\
\hline & ISI (Dubai, UAE & $=0.829$ & РИНЦ (Russia & $=0.156$ & PIF (India) & $=1.940$ \\
\hline & GIF (Australia) & $=0.564$ & ESJI (KZ) & $=8.716$ & IBI (India) & $=4.260$ \\
\hline & JIF & $=1.500$ & SJIF (Morocco & $=5.667$ & OAJI (USA) & $=0.350$ \\
\hline
\end{tabular}

A
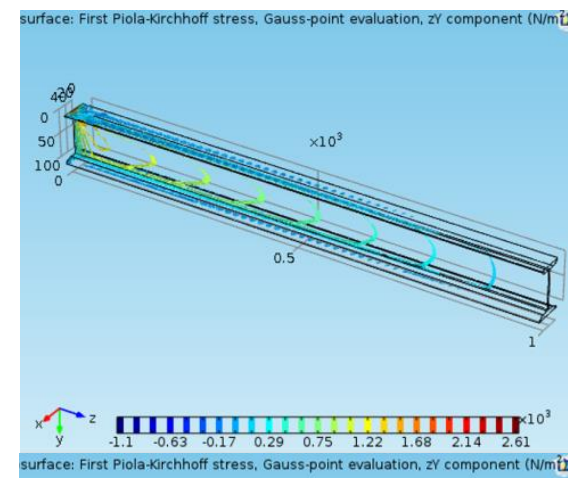

C

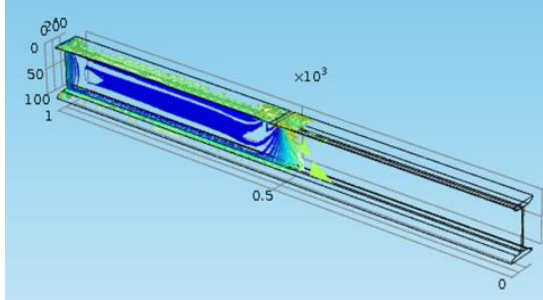

E

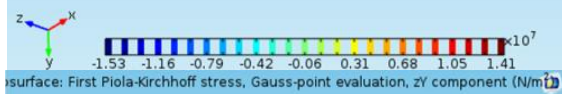

G
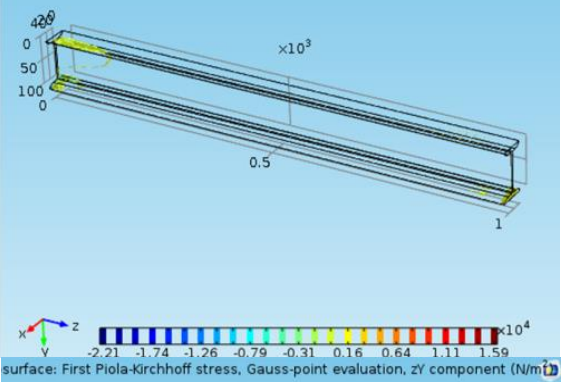

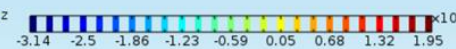
isurface: First Piola-kirchhoff stress, Gauss.point evaluation, $z Y$ component (N/m?

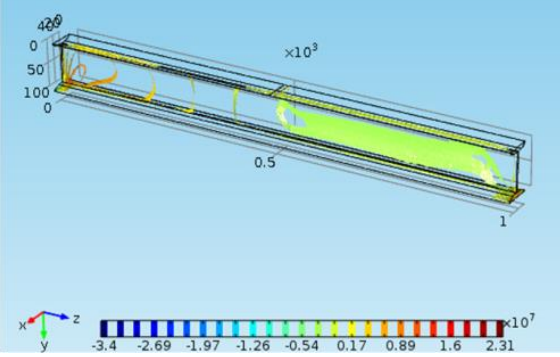

B

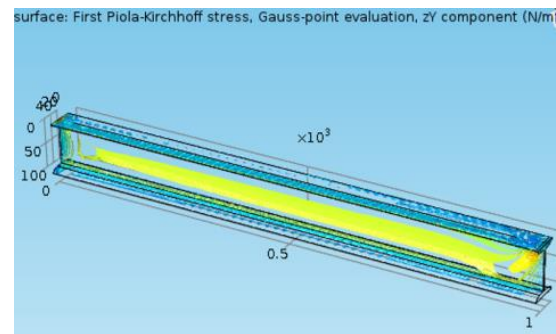

D
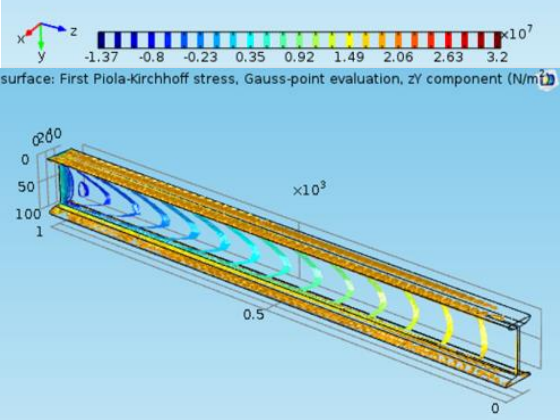

$\mathbf{F}$
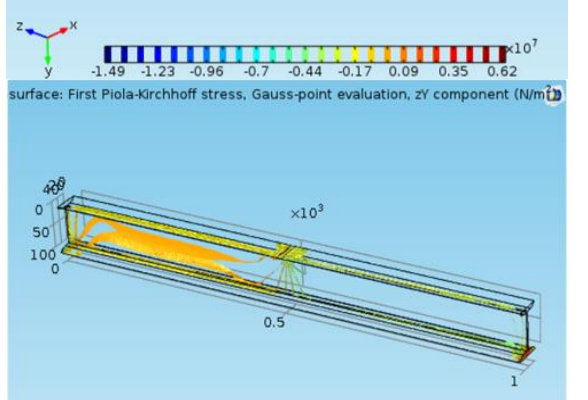

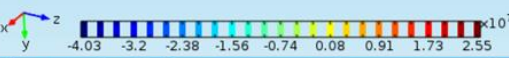

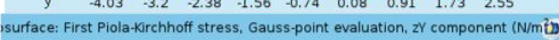$$
\text { surface: First Piola-kirchhoff stress, Gauss-point }
$$

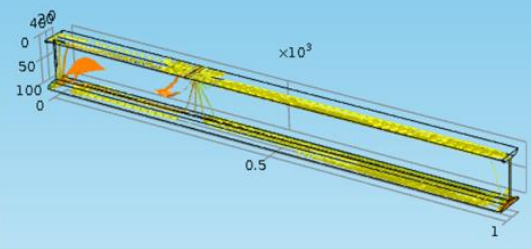

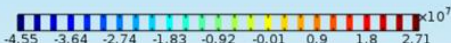

Figure 17 - First Piola-Kirchhoff stress, $\mathrm{zY}$ component: A - the first scheme; $\mathrm{B}$ - the second scheme; $\mathrm{C}$ - the third scheme; D - the fourth scheme; E - the fifth scheme; F - the sixth scheme; $\mathbf{G}$ - the seventh scheme; H the eighth scheme; I - the ninth scheme. 


\begin{tabular}{|c|c|c|c|c|c|c|}
\hline \multirow{4}{*}{ Impact Factor: } & ISRA (India) & $=3.117$ & SIS (USA) & $=0.912$ & ICV (Poland) & $=6.630$ \\
\hline & ISI (Dubai, UAE & $=0.829$ & РИНЦ (Russia & $=0.156$ & PIF (India) & $=1.940$ \\
\hline & GIF (Australia) & $=0.564$ & ESJI (KZ) & $=8.716$ & IBI (India) & $=4.260$ \\
\hline & JIF & $=1.500$ & SJIF (Morocco & $=5.667$ & OAJI (USA) & $=0.350$ \\
\hline
\end{tabular}

A

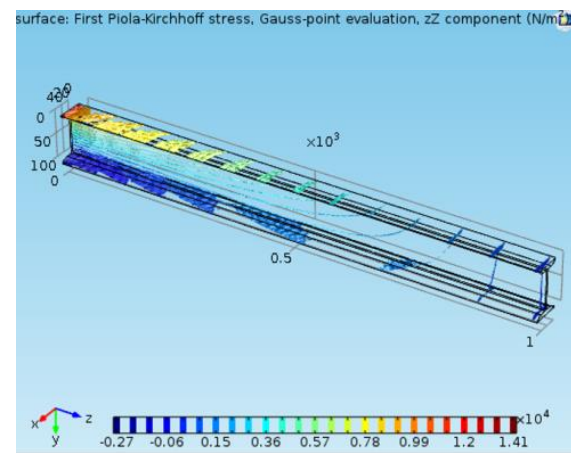

C surface: First Piola-Kirchhoff stress, Gauss-point evaluation, zZ component (N/m

$\mathbf{E}$

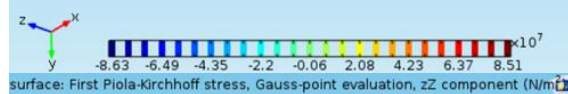

G
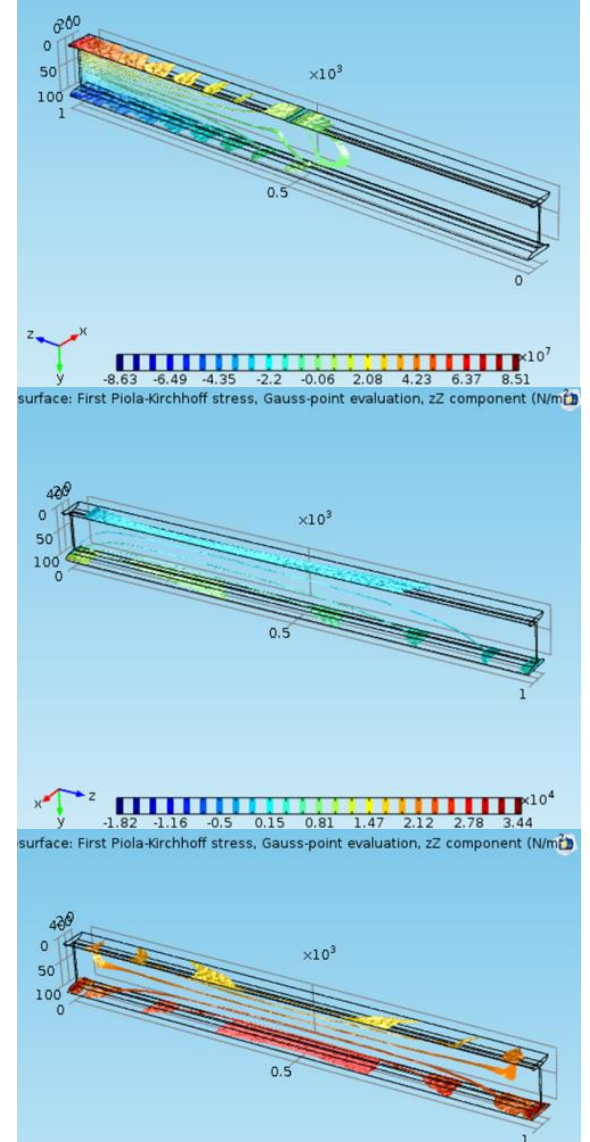

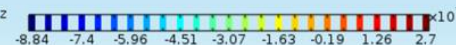
surface: First Piola-Airchhoff stress, Gauss.point evaluation, $z Z$ component (N/mEz)

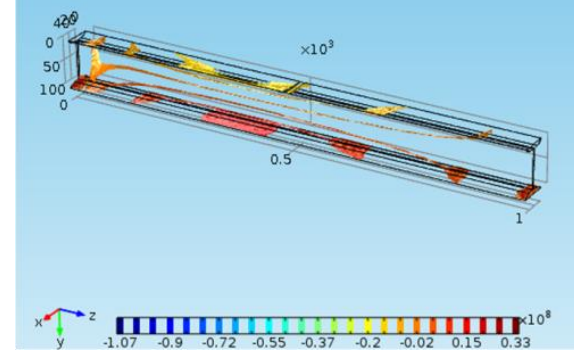

B

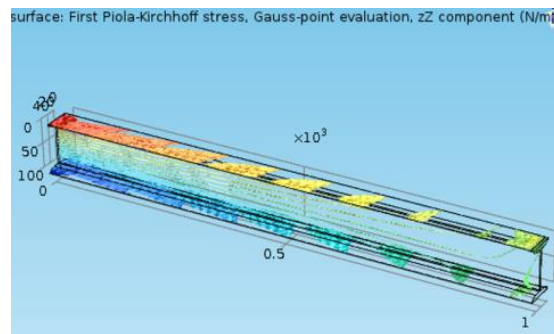

D

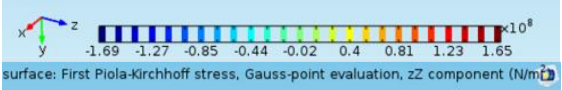

$\mathbf{F}$
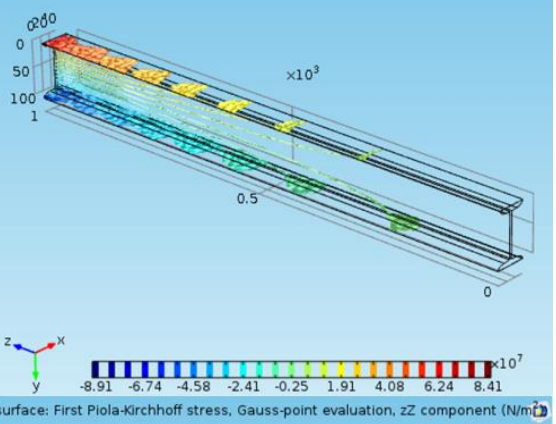

$\mathbf{H}$
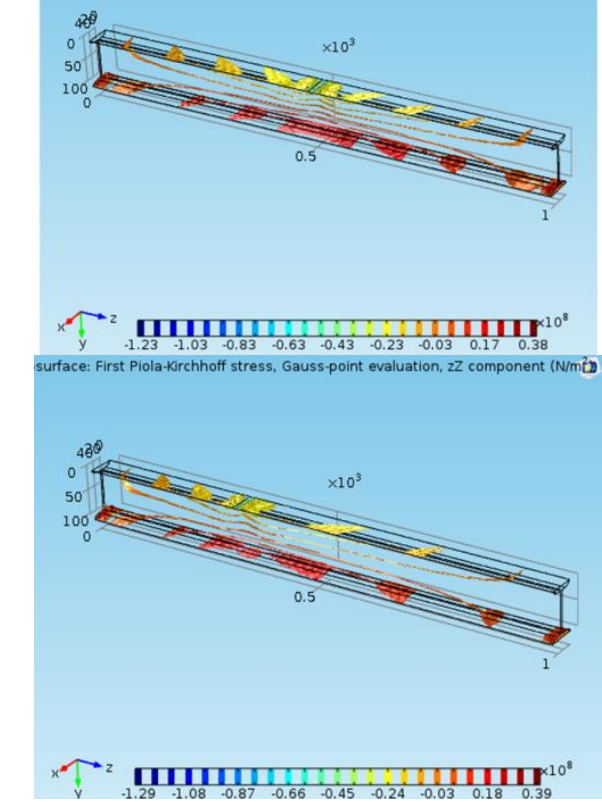

Figure 18 - First Piola-Kirchhoff stress, zZ component: A - the first scheme; $\mathrm{B}$ - the second scheme; $\mathrm{C}$ - the third scheme; D - the fourth scheme; E - the fifth scheme; F - the sixth scheme; $\mathbf{G}$ - the seventh scheme; H the eighth scheme; I - the ninth scheme. 


\begin{tabular}{|c|c|c|c|c|c|c|}
\hline \multirow{4}{*}{ Impact Factor: } & ISRA (India) & $=3.117$ & SIS (USA) & $=0.912$ & ICV (Poland) & $=6.630$ \\
\hline & ISI (Dubai, UAE & $=0.829$ & РИНЦ (Russia & $=0.156$ & PIF (India) & $=1.940$ \\
\hline & GIF (Australia) & $=0.564$ & ESJI (KZ) & $=8.716$ & IBI (India) & $=4.260$ \\
\hline & JIF & $=1.500$ & SJIF (Morocco & $=5.667$ & OAJI (USA) & $=0.350$ \\
\hline
\end{tabular}

A

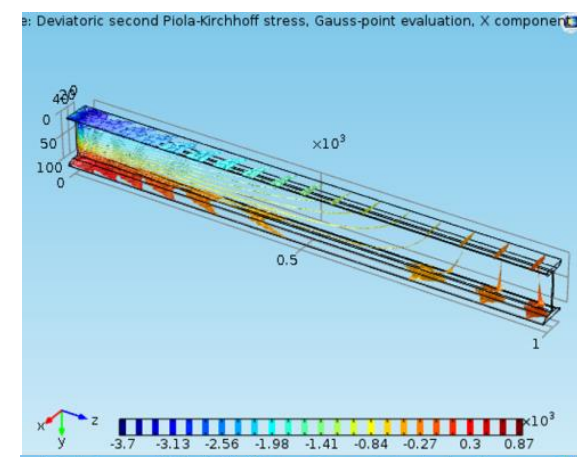

C

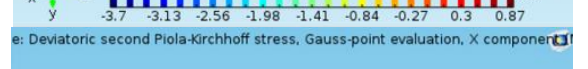

$\mathbf{E}$

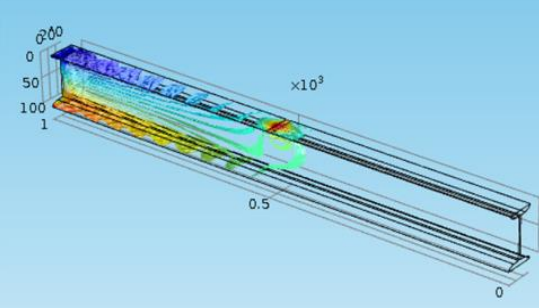

G
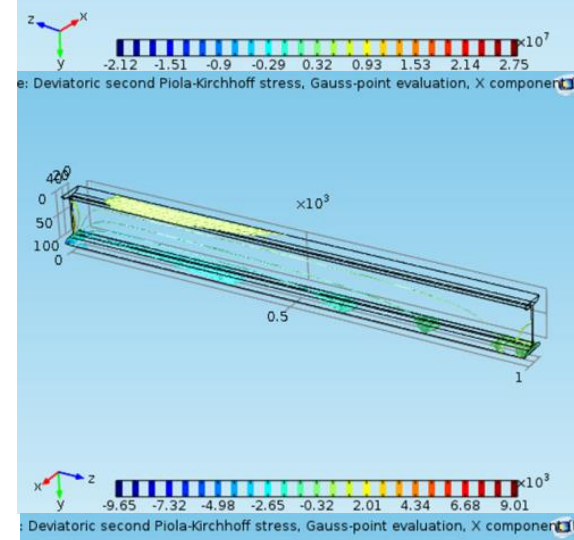

I

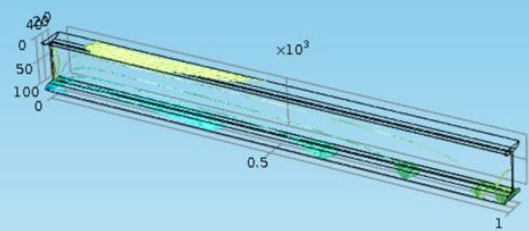

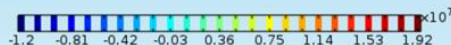

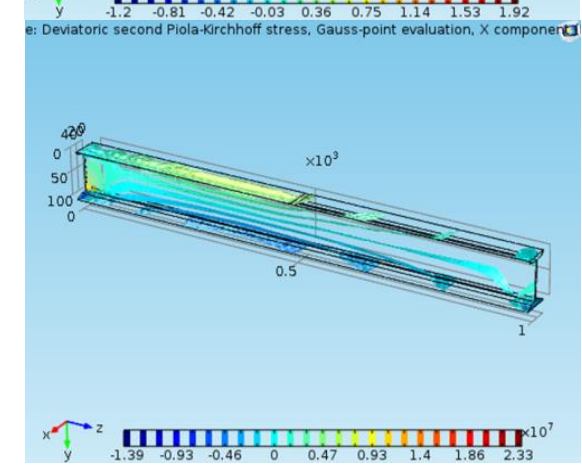

B

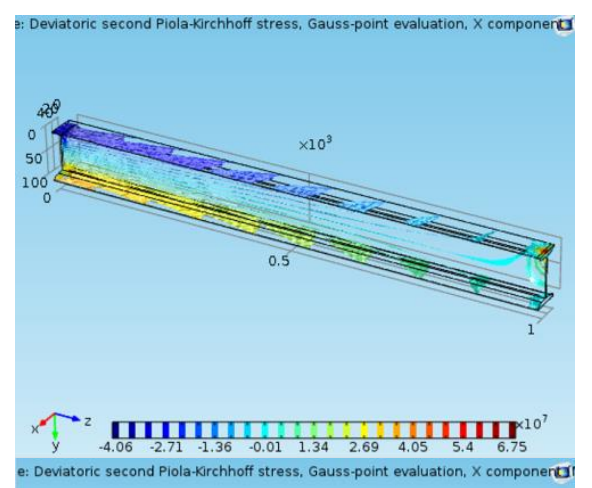

D

$\mathbf{F}$
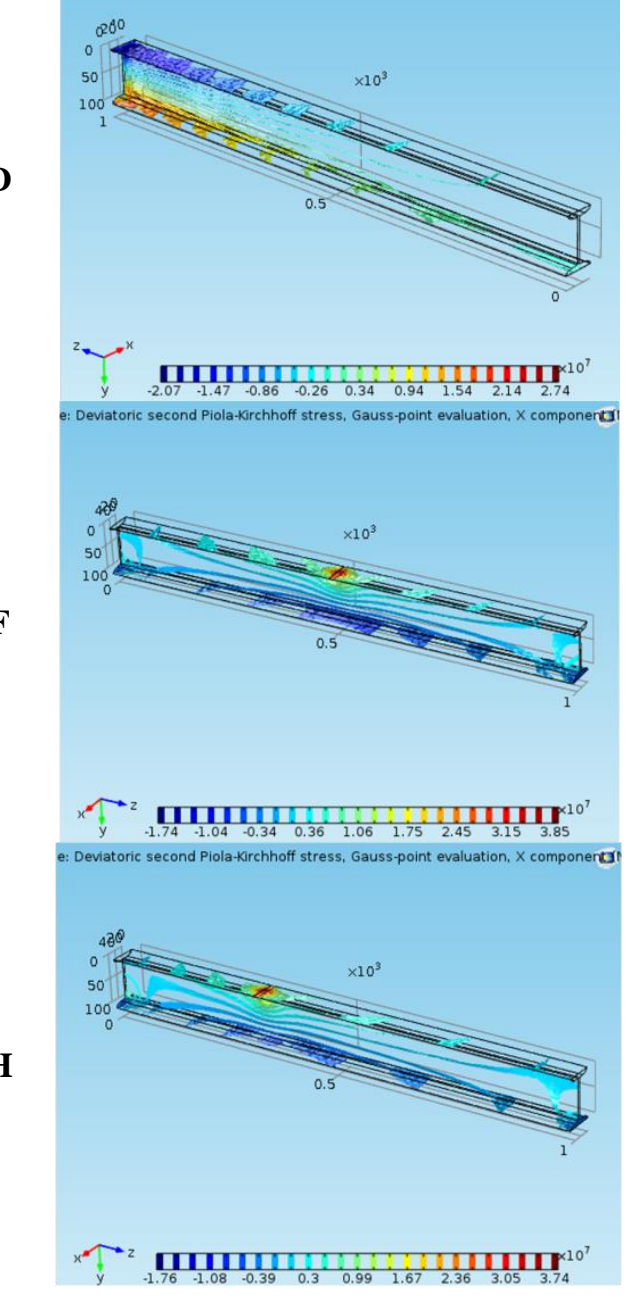

Figure 19 - Deviatoric second Piola-Kirchhoff stress, X component: A - the first scheme; B - the second scheme; $\mathrm{C}$ - the third scheme; D - the fourth scheme; $\mathrm{E}$ - the fifth scheme; $\mathrm{F}$ - the sixth scheme; $\mathrm{G}$ - the seventh scheme; $\mathrm{H}$ - the eighth scheme; I - the ninth scheme. 


\begin{tabular}{|c|c|c|c|c|c|c|}
\hline \multirow{4}{*}{ Impact Factor: } & ISRA (India) & $=3.117$ & SIS (USA) & $=0.912$ & ICV (Poland) & $=6.630$ \\
\hline & ISI (Dubai, UAE & $=0.829$ & РИНЦ (Russia & $=0.156$ & PIF (India) & $=1.940$ \\
\hline & GIF (Australia) & $=0.564$ & ESJI (KZ) & $=8.716$ & IBI (India) & $=4.260$ \\
\hline & JIF & $=1.500$ & SJIF (Morocco & $=5.667$ & OAJI (USA) & $=0.350$ \\
\hline
\end{tabular}

A

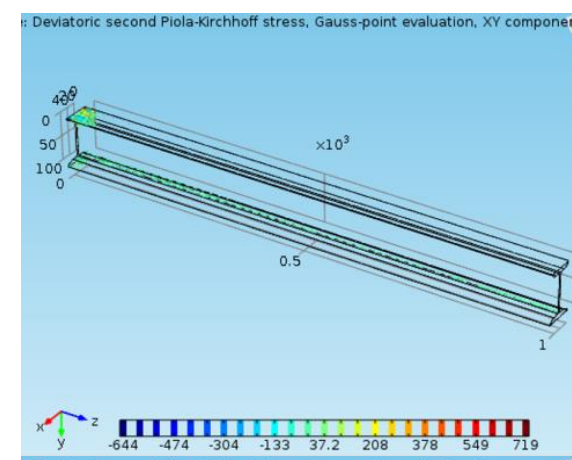

C

$\mathbf{E}$
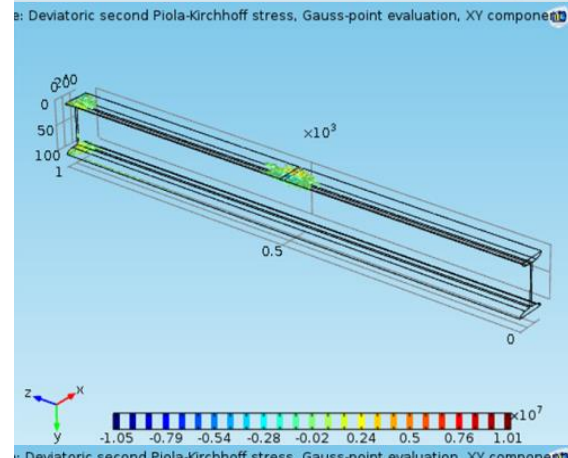

G
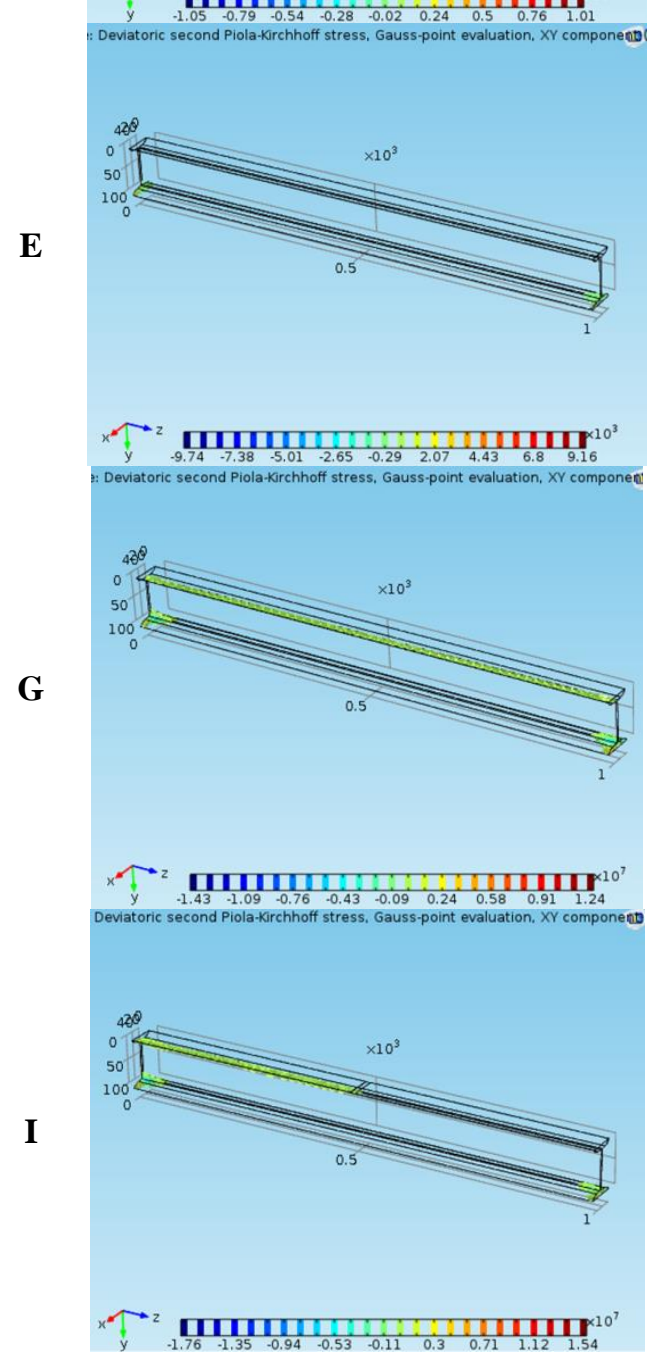

B

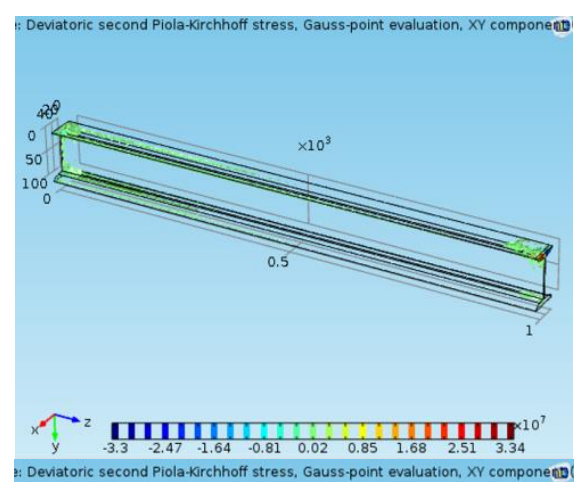

D

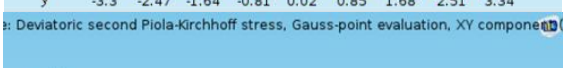

$\mathbf{F}$
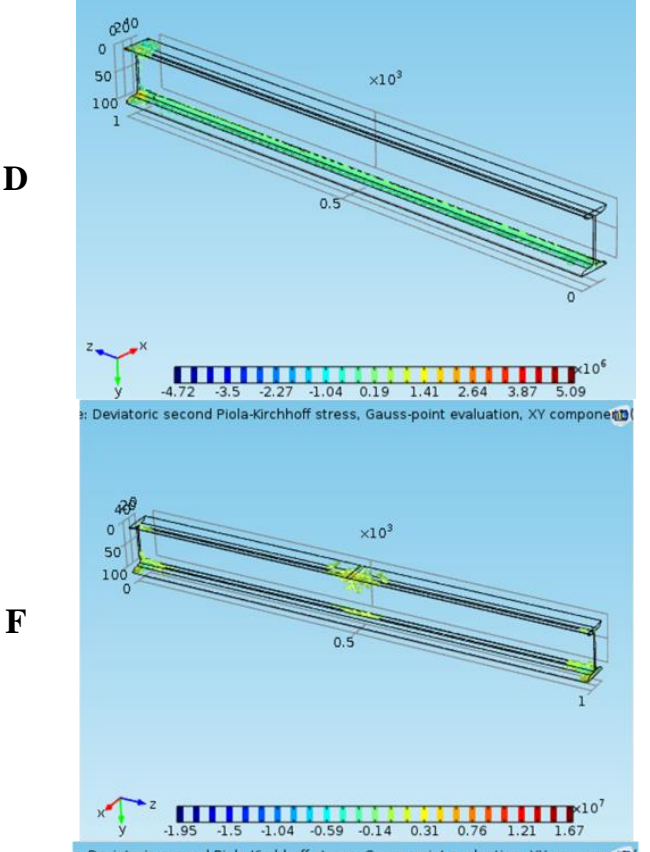

$\mathbf{H}$

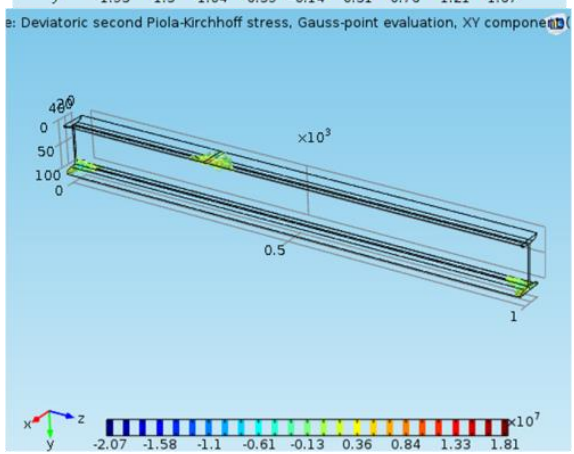

Figure 20 - Deviatoric second Piola-Kirchhoff stress, XY component: A - the first scheme; B - the second scheme; $\mathrm{C}$ - the third scheme; D - the fourth scheme; $\mathrm{E}$ - the fifth scheme; $\mathrm{F}$ - the sixth scheme; $\mathbf{G}$ - the seventh scheme; $\mathrm{H}$ - the eighth scheme; I - the ninth scheme. 


\begin{tabular}{|c|c|c|c|c|c|c|}
\hline \multirow{4}{*}{ Impact Factor: } & ISRA (India) & $=3.117$ & SIS (USA) & $=0.912$ & ICV (Poland) & $=6.630$ \\
\hline & ISI (Dubai, UAE & $=0.829$ & РИНЦ (Russia & $=0.156$ & PIF (India) & $=1.940$ \\
\hline & GIF (Australia) & $=0.564$ & ESJI (KZ) & $=8.716$ & IBI (India) & $=4.260$ \\
\hline & JIF & $=1.500$ & SJIF (Morocco & $=5.667$ & OAJI (USA) & $=0.350$ \\
\hline
\end{tabular}

A

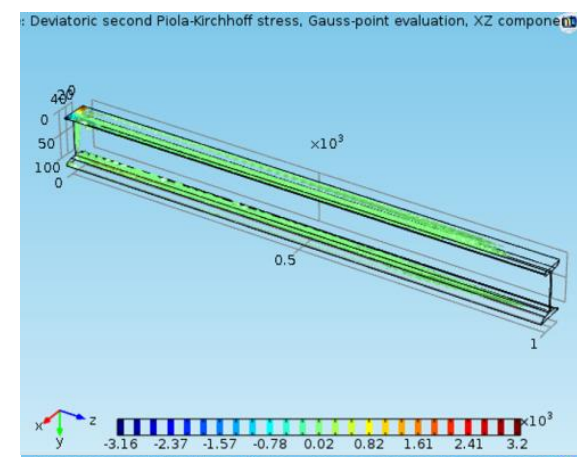

C

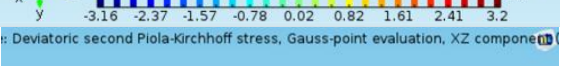

$\mathbf{E}$
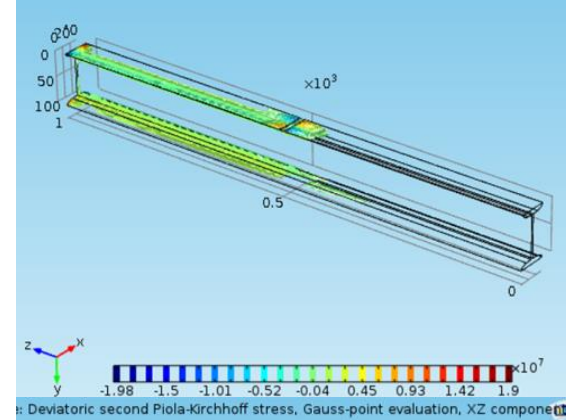

G
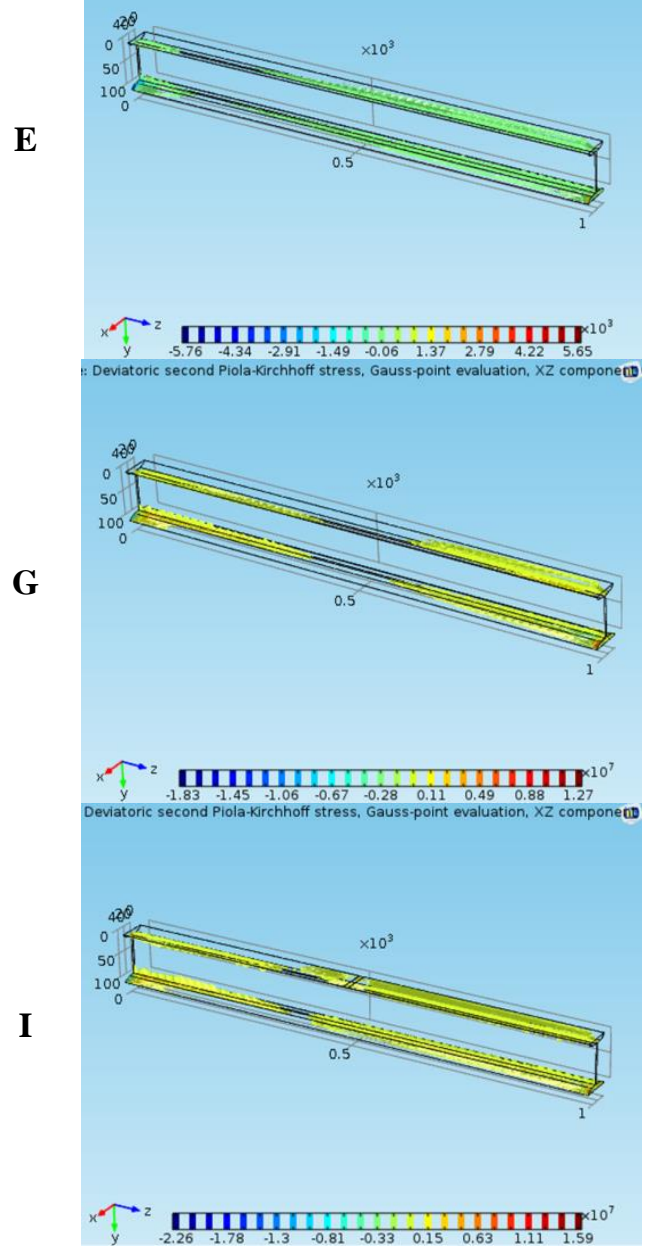

I
B

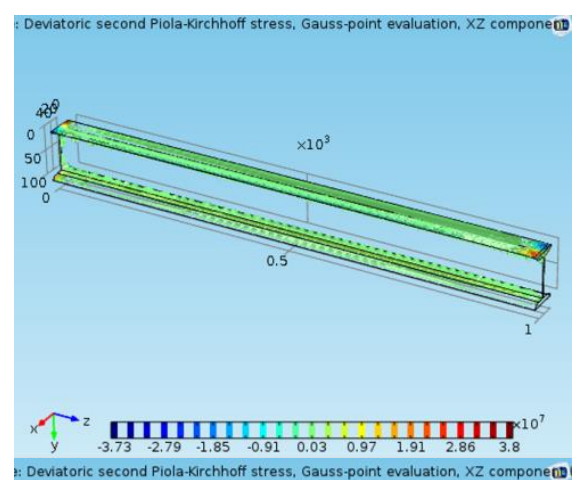

D

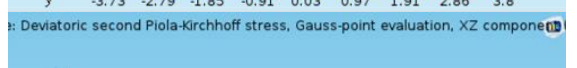

$\mathbf{F}$
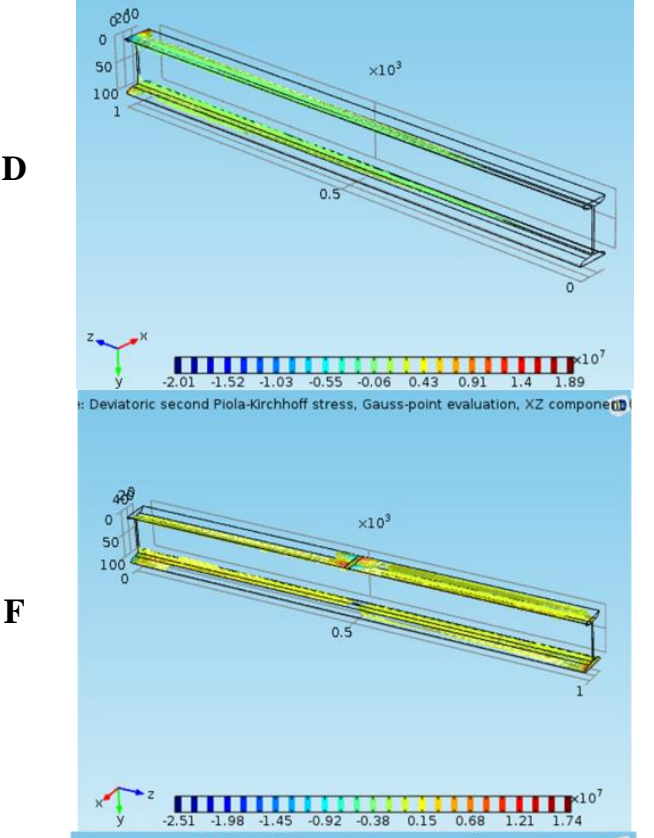

$\mathbf{H}$

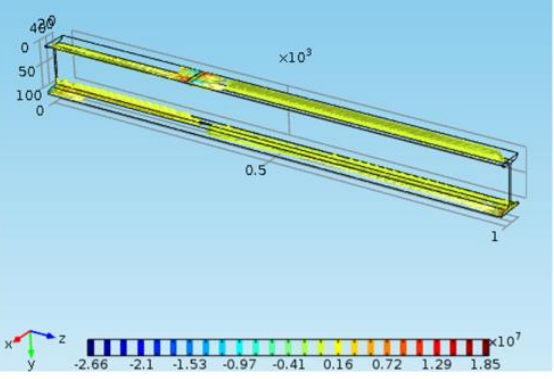

Figure 21 - Deviatoric second Piola-Kirchhoff stress, XZ component: A - the first scheme; $B$ - the second scheme; $\mathrm{C}$ - the third scheme; $\mathrm{D}$ - the fourth scheme; $\mathrm{E}$ - the fifth scheme; $\mathrm{F}$ - the sixth scheme; $\mathrm{G}$ - the seventh scheme; $\mathrm{H}$ - the eighth scheme; I - the ninth scheme. 


\begin{tabular}{|c|c|c|c|c|c|c|}
\hline \multirow{4}{*}{ Impact Factor: } & ISRA (India) & $=3.117$ & SIS (USA) & $=0.912$ & ICV (Poland) & $=6.630$ \\
\hline & ISI (Dubai, UAE & $=0.829$ & РИНЦ (Russia & $=0.156$ & PIF (India) & $=1.940$ \\
\hline & GIF (Australia) & $=0.564$ & ESJI (KZ) & $=8.716$ & IBI (India) & $=4.260$ \\
\hline & JIF & $=1.500$ & SJIF (Morocco & $=5.667$ & OAJI (USA) & $=0.350$ \\
\hline
\end{tabular}

A

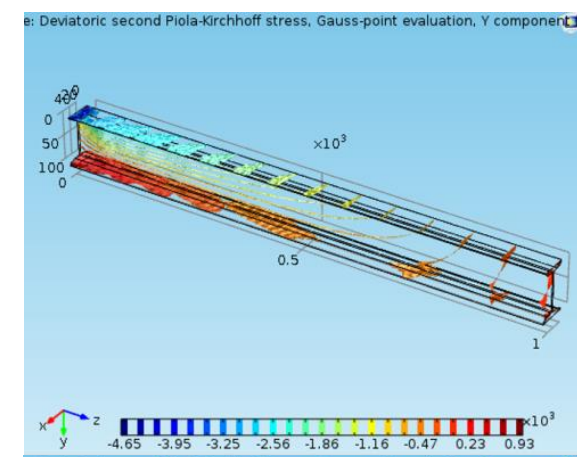

C

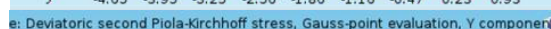

$\mathbf{E}$
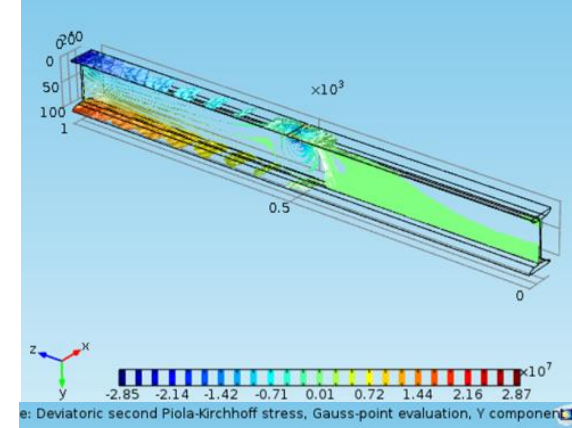

G
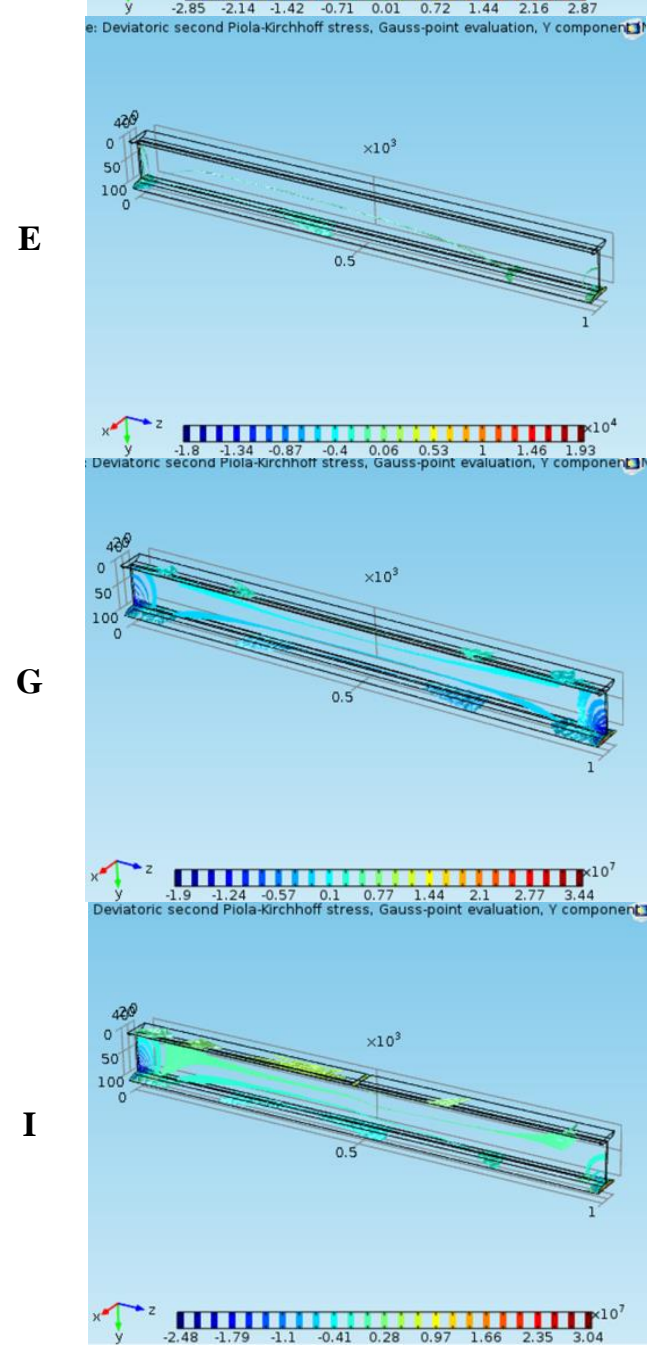

B

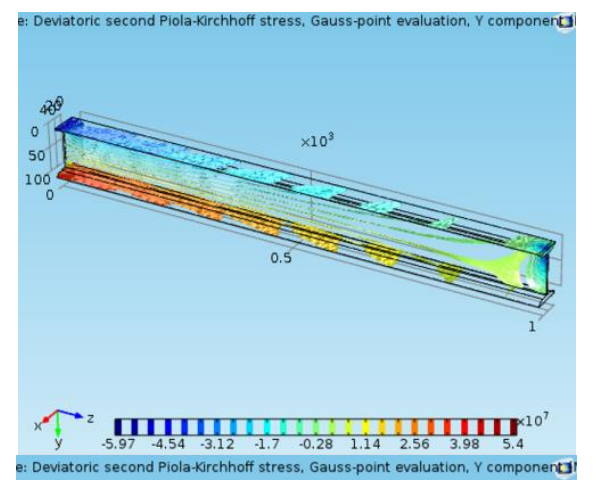

D

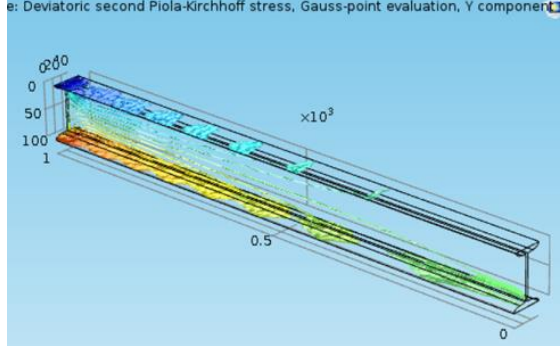

$\mathbf{F}$

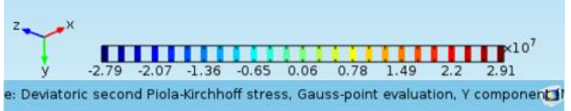

$\mathbf{H}$
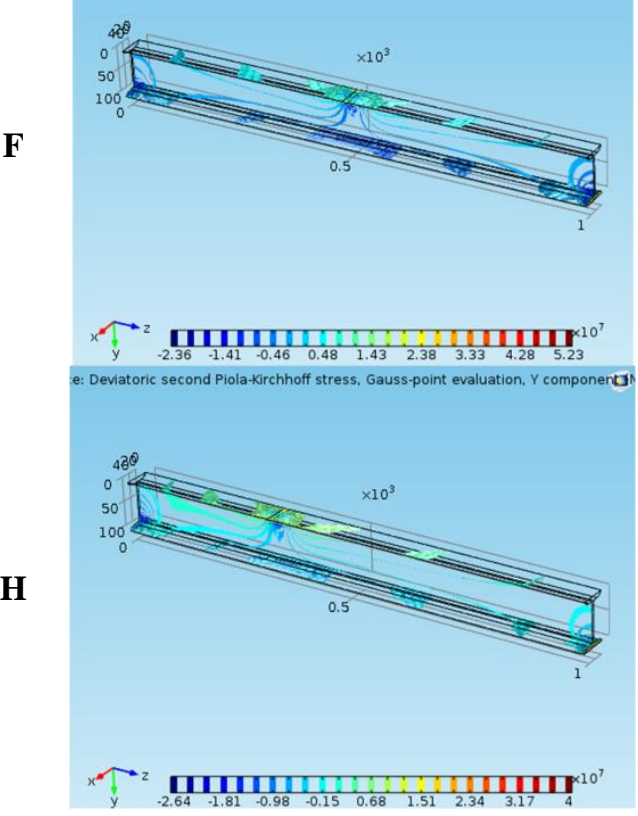

Figure 22 - Deviatoric second Piola-Kirchhoff stress, Y component: A - the first scheme; B - the second scheme; $\mathrm{C}$ - the third scheme; D - the fourth scheme; $\mathrm{E}$ - the fifth scheme; $\mathrm{F}$ - the sixth scheme; $\mathrm{G}$ - the seventh scheme; $\mathrm{H}$ - the eighth scheme; I - the ninth scheme. 


\begin{tabular}{|c|c|c|c|c|c|c|}
\hline \multirow{4}{*}{ Impact Factor: } & ISRA (India) & $=3.117$ & SIS (USA) & $=0.912$ & ICV (Poland) & $=6.630$ \\
\hline & ISI (Dubai, UAE & $=0.829$ & РИНЦ (Russia & $=0.156$ & PIF (India) & $=1.940$ \\
\hline & GIF (Australia) & $=0.564$ & ESJI (KZ) & $=8.716$ & IBI (India) & $=4.260$ \\
\hline & JIF & $=1.500$ & SJIF (Morocco & $=5.667$ & OAJI (USA) & $=0.350$ \\
\hline
\end{tabular}

A

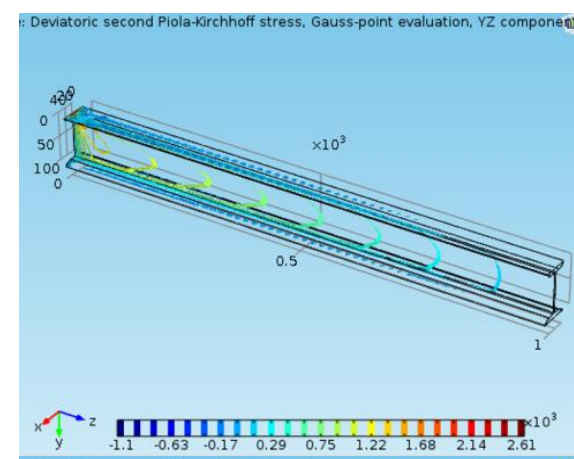

C

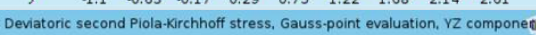

E
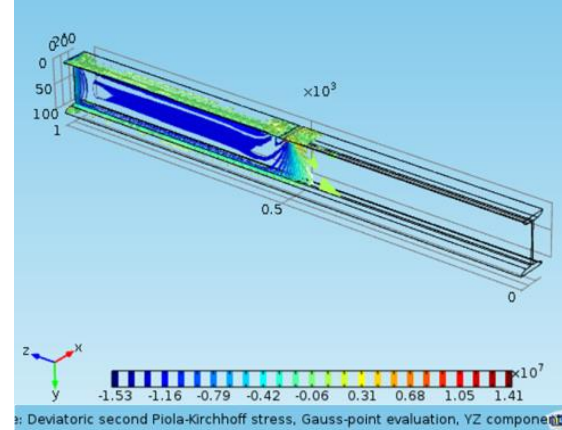

G
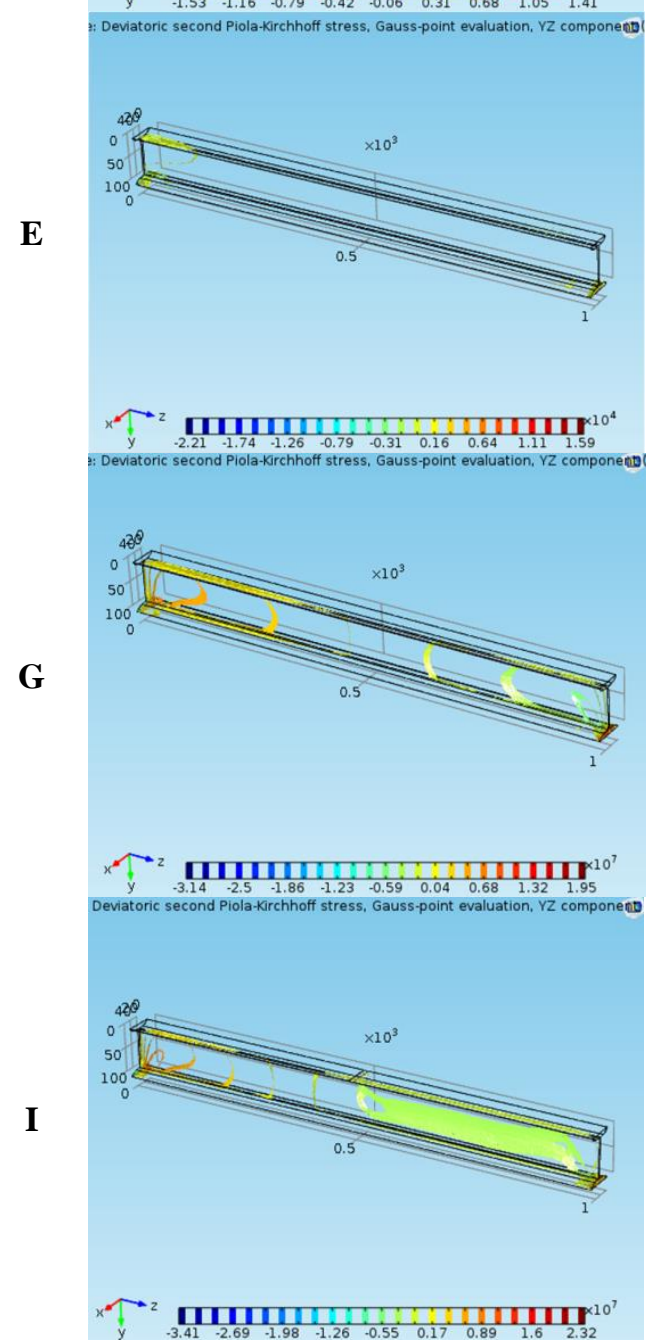

B

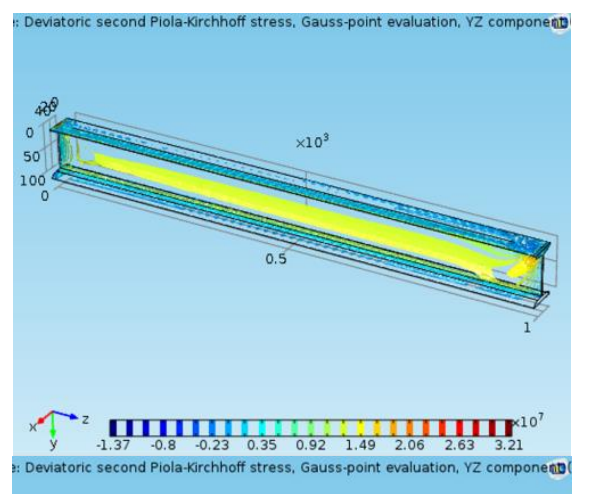

D

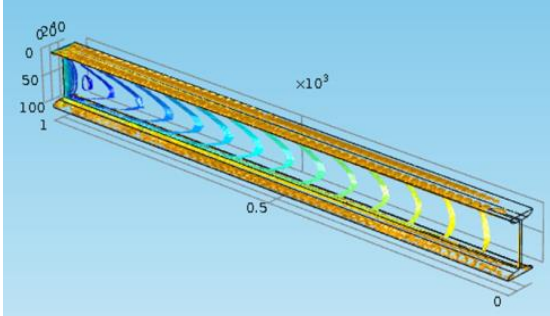

$\mathbf{F}$

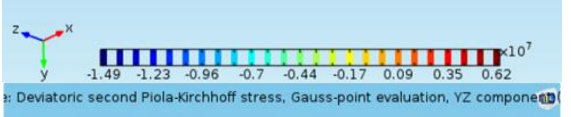

$\mathbf{H}$
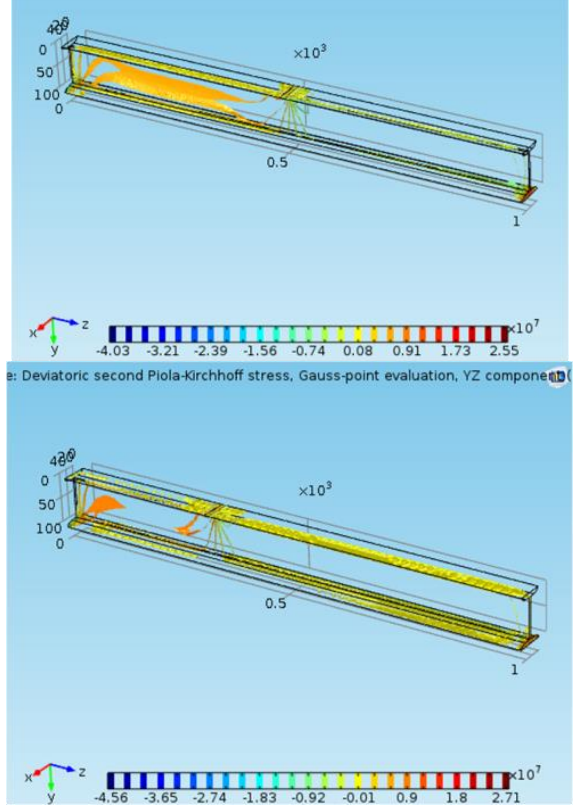

Figure 23 - Deviatoric second Piola-Kirchhoff stress, YZ component: A - the first scheme; B - the second scheme; $\mathrm{C}$ - the third scheme; D - the fourth scheme; $\mathrm{E}$ - the fifth scheme; $\mathrm{F}$ - the sixth scheme; $\mathbf{G}$ - the seventh scheme; $\mathrm{H}$ - the eighth scheme; I - the ninth scheme. 


\begin{tabular}{|c|c|c|c|c|c|c|}
\hline \multirow{4}{*}{ Impact Factor: } & ISRA (India) & $=3.117$ & SIS (USA) & $=0.912$ & ICV (Poland) & $=6.630$ \\
\hline & ISI (Dubai, UAE & $=0.829$ & РИНЦ (Russia & $=0.156$ & PIF (India) & $=1.940$ \\
\hline & GIF (Australia) & $=0.564$ & ESJI (KZ) & $=8.716$ & IBI (India) & $=4.260$ \\
\hline & JIF & $=1.500$ & SJIF (Morocco & $=5.667$ & OAJI (USA) & $=0.350$ \\
\hline
\end{tabular}

A

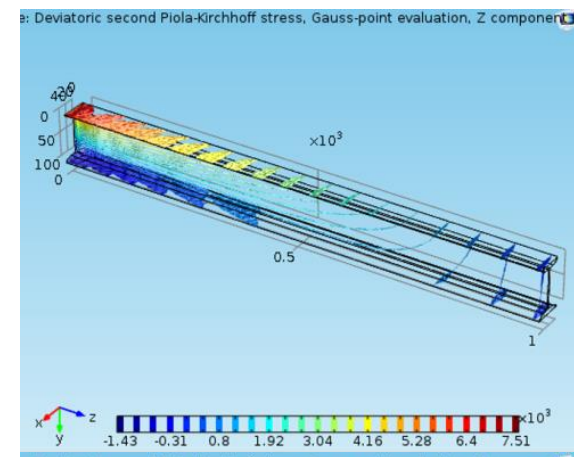

C
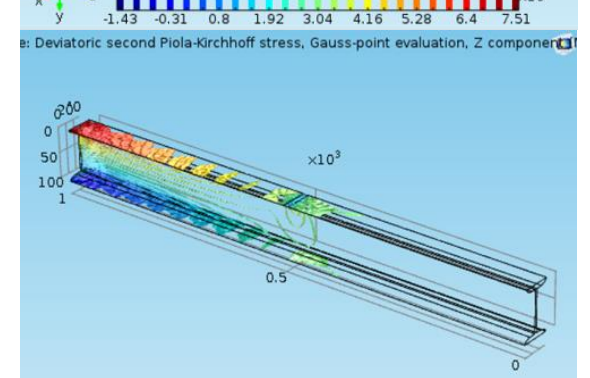

$\mathbf{E}$

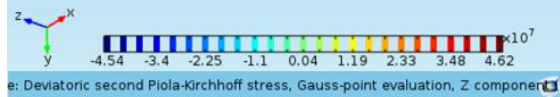

G
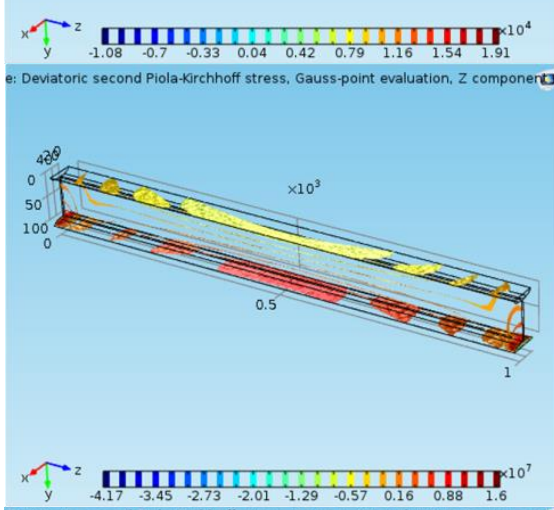

I

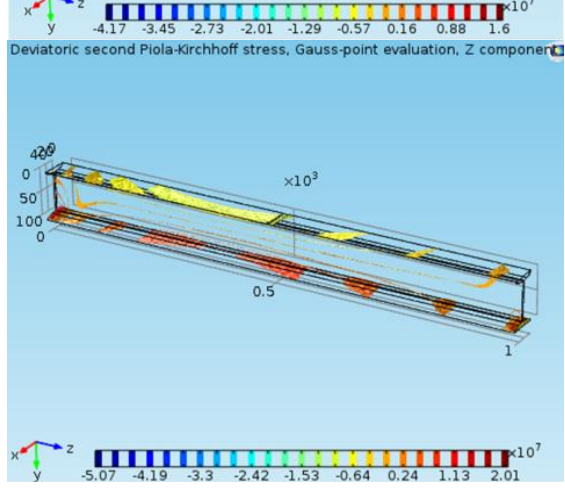

B

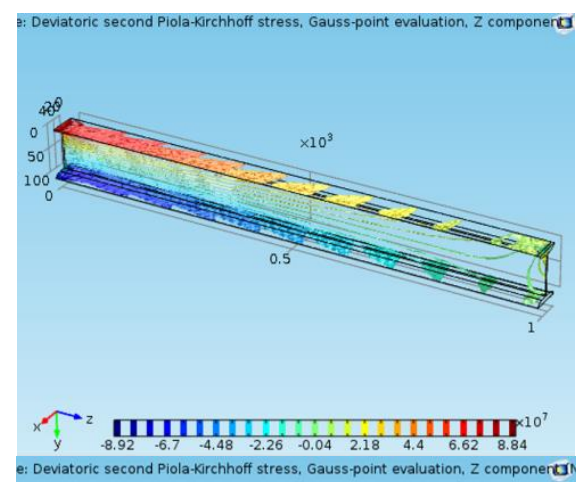

D

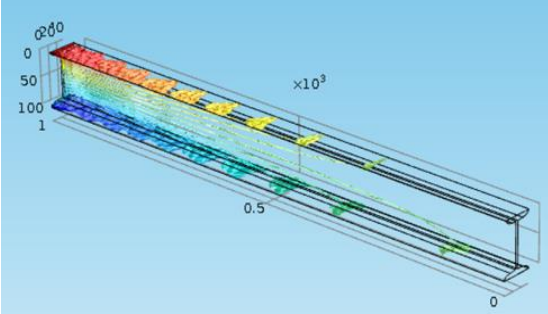

$\mathbf{F}$

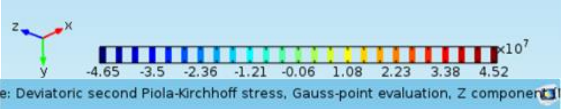

$\mathbf{H}$

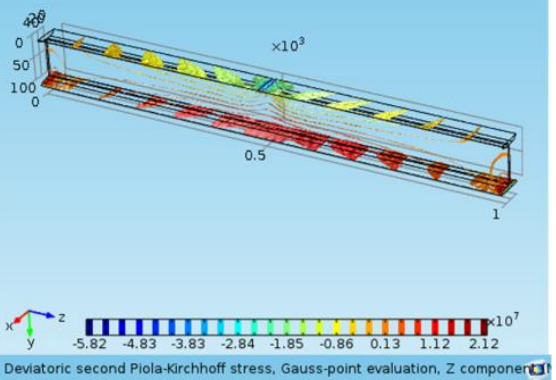

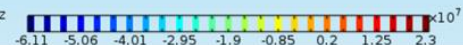

Figure 24 - Deviatoric second Piola-Kirchhoff stress, $Z$ component: $A$ - the first scheme; $B$ - the second scheme; $\mathrm{C}$ - the third scheme; D - the fourth scheme; $\mathrm{E}$ - the fifth scheme; $\mathrm{F}$ - the sixth scheme; $\mathrm{G}$ - the seventh scheme; $\mathrm{H}$ - the eighth scheme; I - the ninth scheme. 


\begin{tabular}{|c|c|c|c|c|c|c|}
\hline \multirow{4}{*}{ Impact Factor: } & ISRA (India) & $=3.117$ & SIS (USA) & $=0.912$ & ICV (Poland) & $=6.630$ \\
\hline & ISI (Dubai, UAE & $=0.829$ & РИНЦ (Russia & $=0.156$ & PIF (India) & $=1.940$ \\
\hline & GIF (Australia) & $=0.564$ & ESJI (KZ) & $=8.716$ & IBI (India) & $=4.260$ \\
\hline & JIF & $=1.500$ & SJIF (Morocco & $=5.667$ & OAJI (USA) & $=0.350$ \\
\hline
\end{tabular}

A

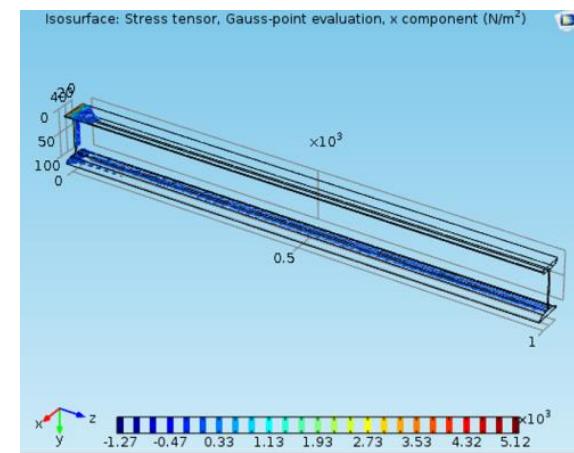

C

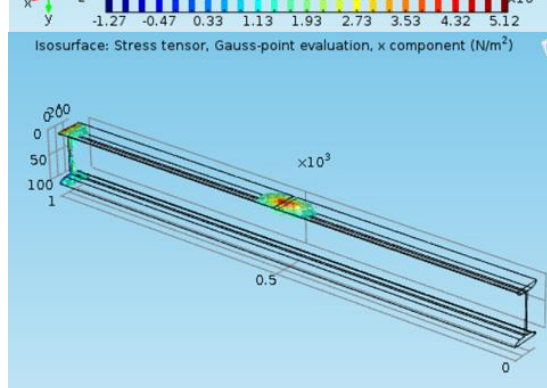

E

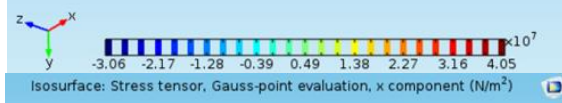

G
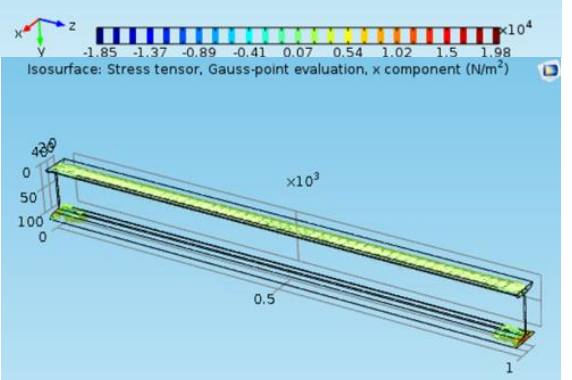

I

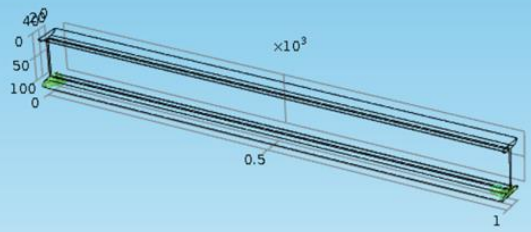

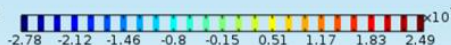
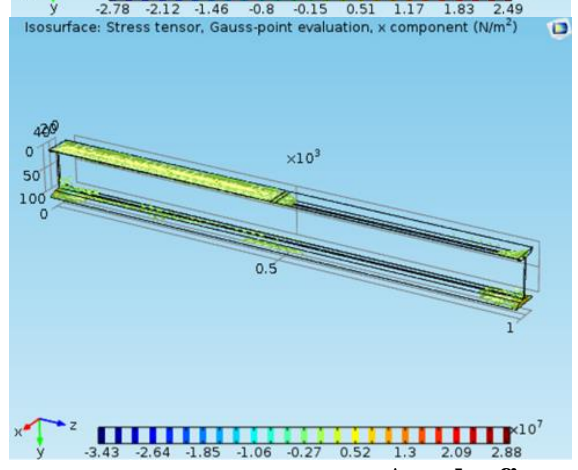

B

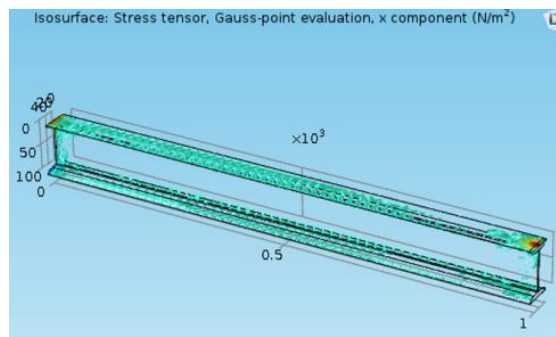

D

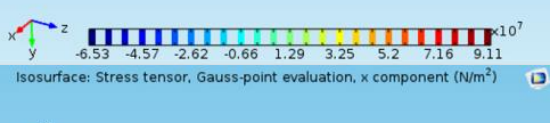

F

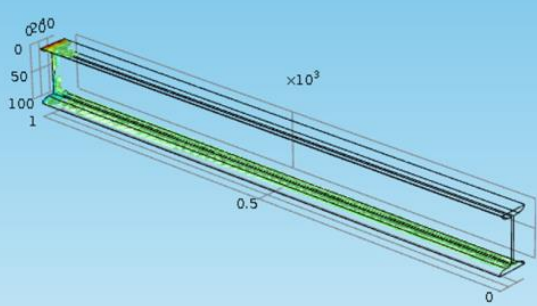

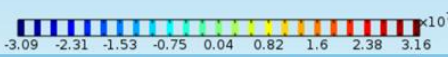
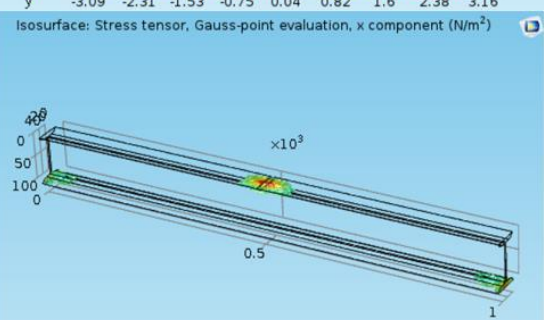

H
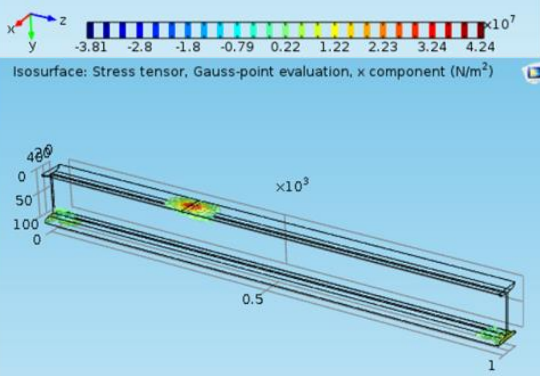

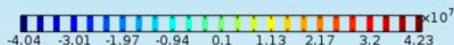

Figure 25 - Stress tensor, $x$ component: A - the first scheme; $B$ - the second scheme; $C$ - the third scheme; D - the fourth scheme; $\mathrm{E}$ - the fifth scheme; $\mathrm{F}$ - the sixth scheme; $\mathrm{G}$ - the seventh scheme; $\mathrm{H}$ - the eighth scheme; I - the ninth scheme. 


\begin{tabular}{|c|c|c|c|c|c|c|}
\hline \multirow{4}{*}{ Impact Factor: } & ISRA (India) & $=3.117$ & SIS (USA) & $=0.912$ & ICV (Poland) & $=6.630$ \\
\hline & ISI (Dubai, UAE & $=0.829$ & РИНЦ (Russia & $=0.156$ & PIF (India) & $=1.940$ \\
\hline & GIF (Australia) & $=0.564$ & ESJI (KZ) & $=8.716$ & IBI (India) & $=4.260$ \\
\hline & JIF & $=1.500$ & SJIF (Morocco & $=5.667$ & OAJI (USA) & $=0.350$ \\
\hline
\end{tabular}

$\mathbf{A}$

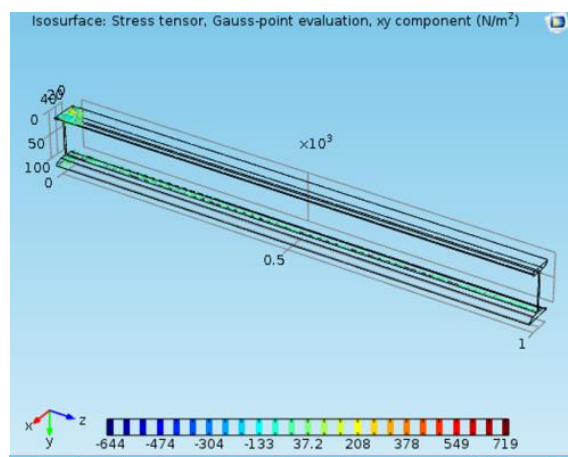

C

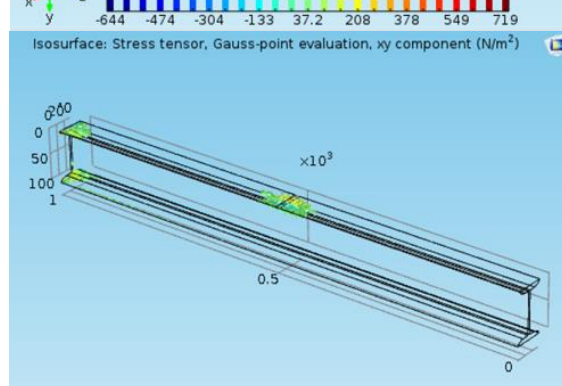

$\mathbf{E}$

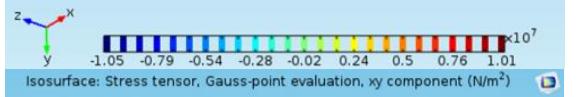

G
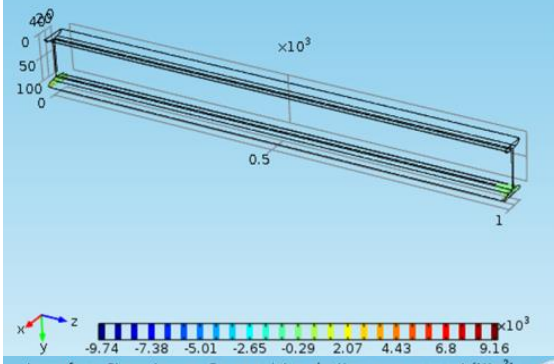

I

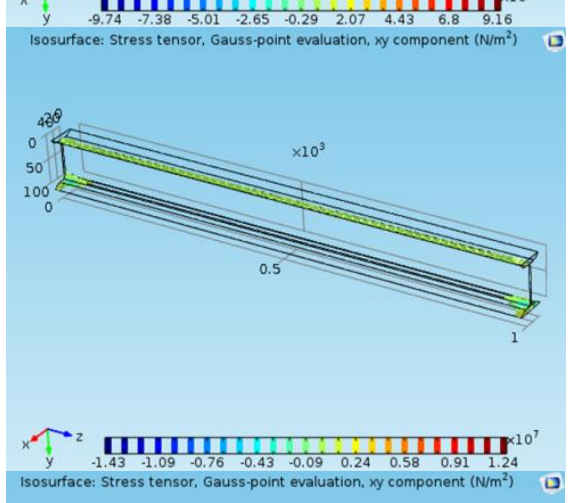

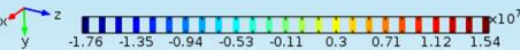

B

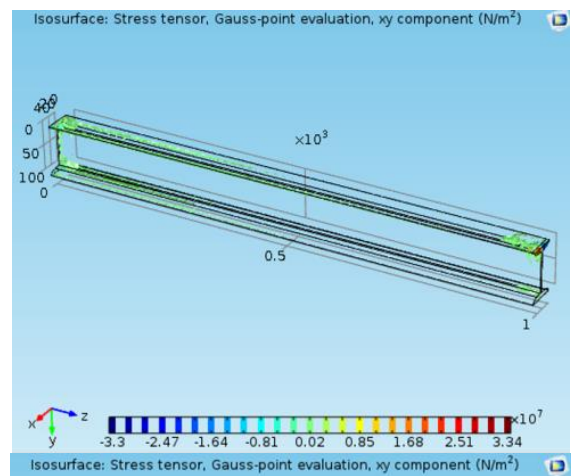

D

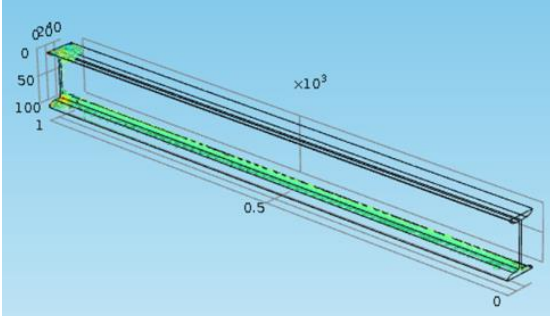

F

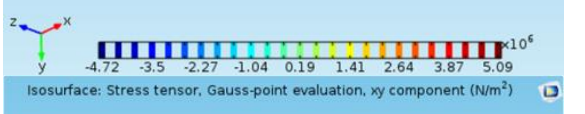

H
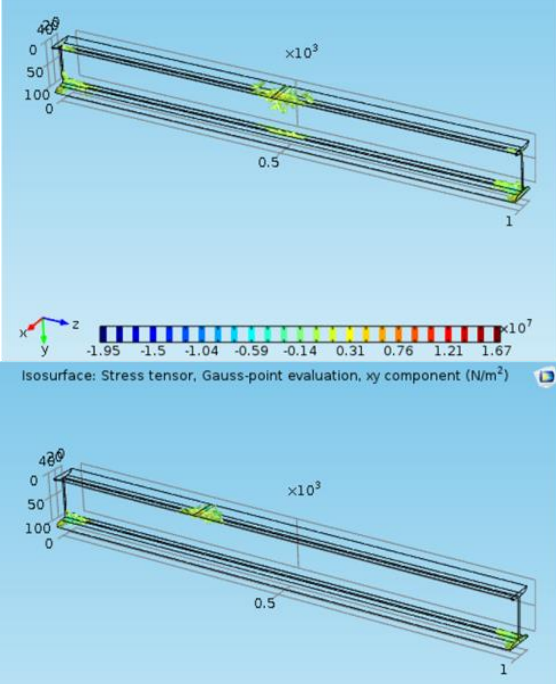

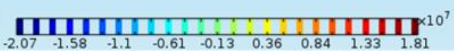

Figure 26 - Stress tensor, $x y$ component: A - the first scheme; $B$ - the second scheme; $C$ - the third scheme; D - the fourth scheme; $\mathbf{E}$ - the fifth scheme; $\mathbf{F}$ - the sixth scheme; $\mathbf{G}$ - the seventh scheme; $\mathbf{H}$ - the eighth scheme; I - the ninth scheme. 


\begin{tabular}{|c|c|c|c|c|c|c|}
\hline \multirow{4}{*}{ Impact Factor: } & ISRA (India) & $=3.117$ & SIS (USA) & $=0.912$ & ICV (Poland) & $=6.630$ \\
\hline & ISI (Dubai, UAE & $=0.829$ & РИНЦ (Russia & $=0.156$ & PIF (India) & $=1.940$ \\
\hline & GIF (Australia) & $=0.564$ & ESJI (KZ) & $=8.716$ & IBI (India) & $=4.260$ \\
\hline & JIF & $=1.500$ & SJIF (Morocco & $=5.667$ & OAJI (USA) & $=0.350$ \\
\hline
\end{tabular}

A

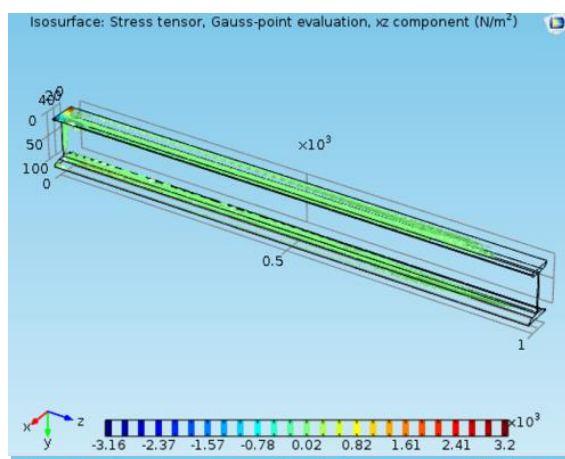

C

$\mathbf{E}$

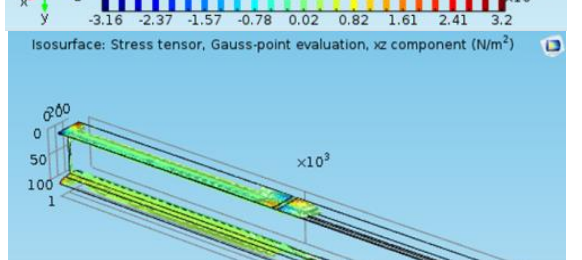

G

I

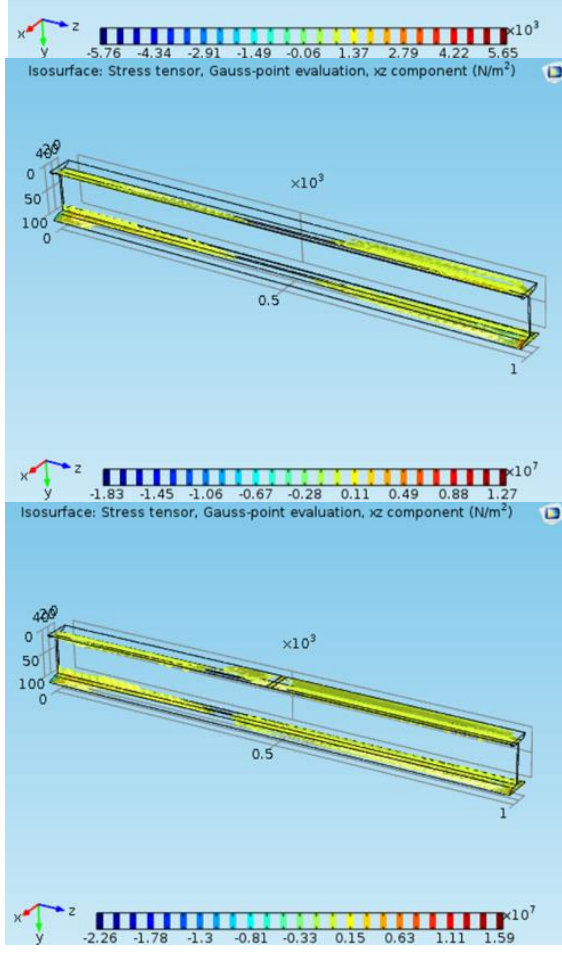

B

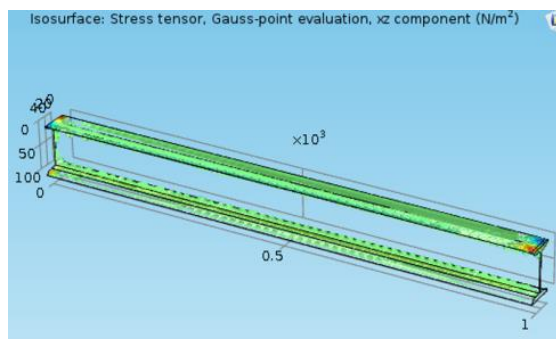

D

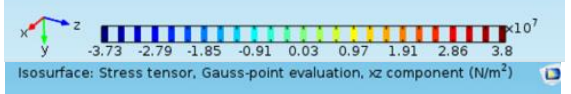

D
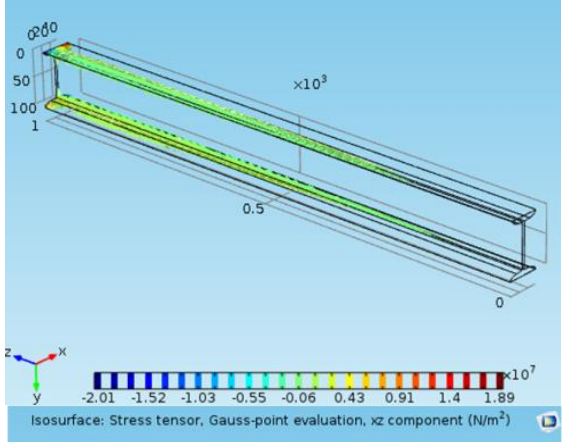

$\mathbf{H}$
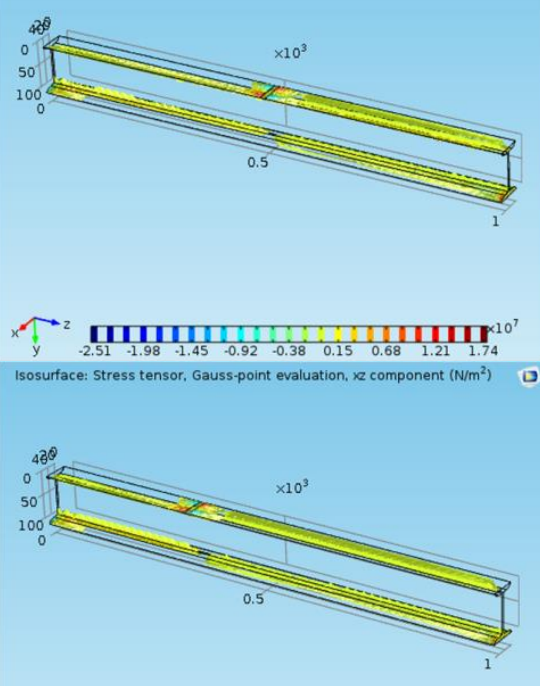

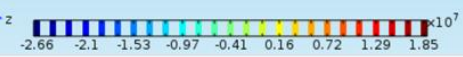

Figure 27 - Stress tensor, $x z$ component: A - the first scheme; B - the second scheme; $C$ - the third scheme; D - the fourth scheme; $\mathbf{E}$ - the fifth scheme; $\mathbf{F}$ - the sixth scheme; $\mathbf{G}$ - the seventh scheme; $\mathbf{H}$ - the eighth scheme; I - the ninth scheme. 


\begin{tabular}{|c|c|c|c|c|c|c|}
\hline \multirow{4}{*}{ Impact Factor: } & ISRA (India) & $=3.117$ & SIS (USA) & $=0.912$ & ICV (Poland) & $=6.630$ \\
\hline & ISI (Dubai, UAE & $=0.829$ & РИНЦ (Russia & $=0.156$ & PIF (India) & $=1.940$ \\
\hline & GIF (Australia) & $=0.564$ & ESJI (KZ) & $=8.716$ & IBI (India) & $=4.260$ \\
\hline & JIF & $=1.500$ & SJIF (Morocco & $=5.667$ & OAJI (USA) & $=0.350$ \\
\hline
\end{tabular}

A

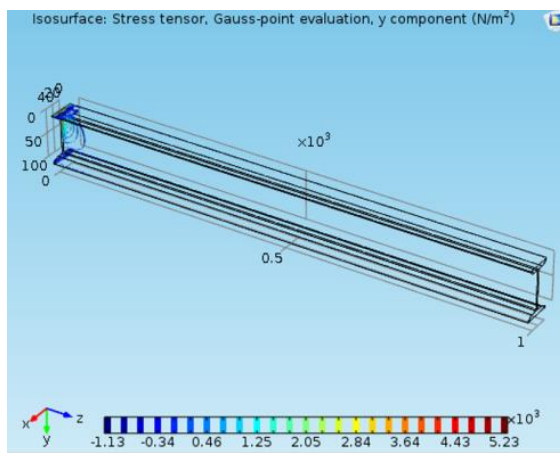

C

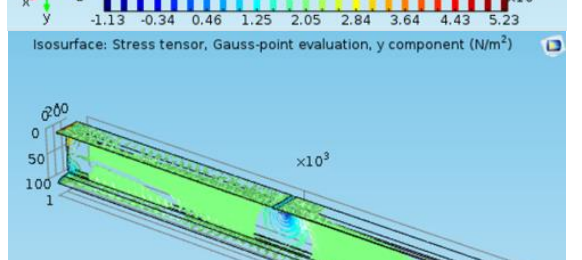

$\mathbf{E}$

G
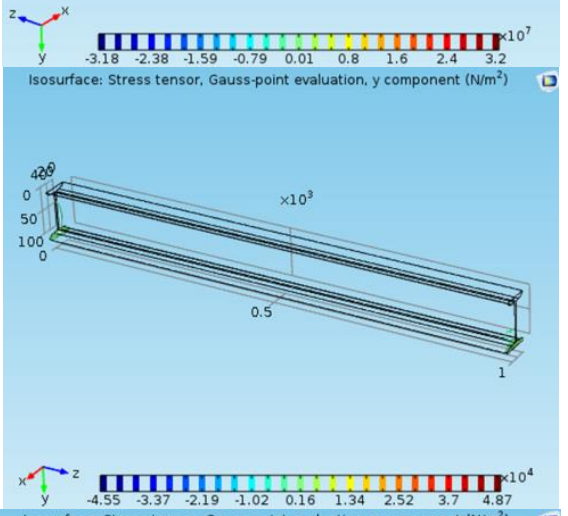

I

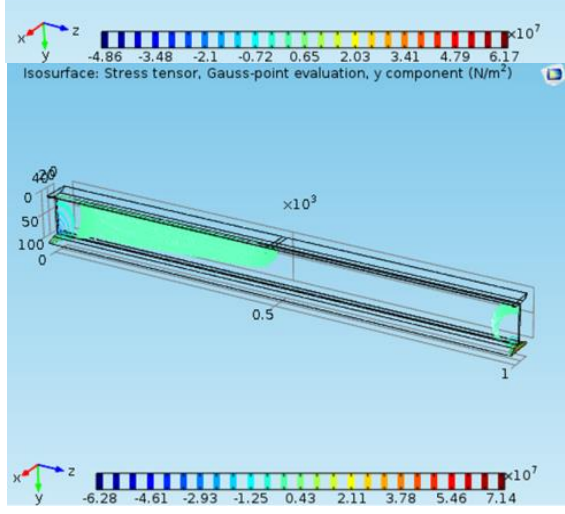

B

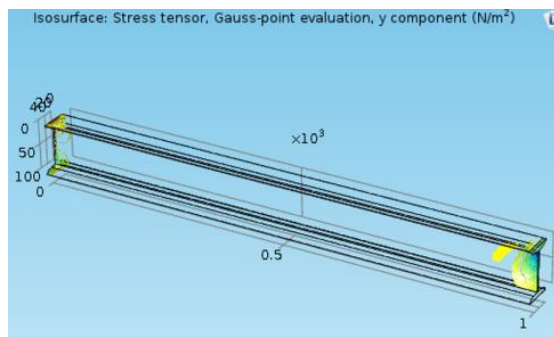

D

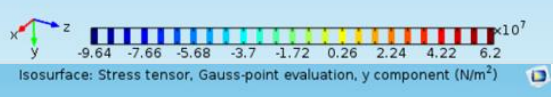

$\mathbf{F}$
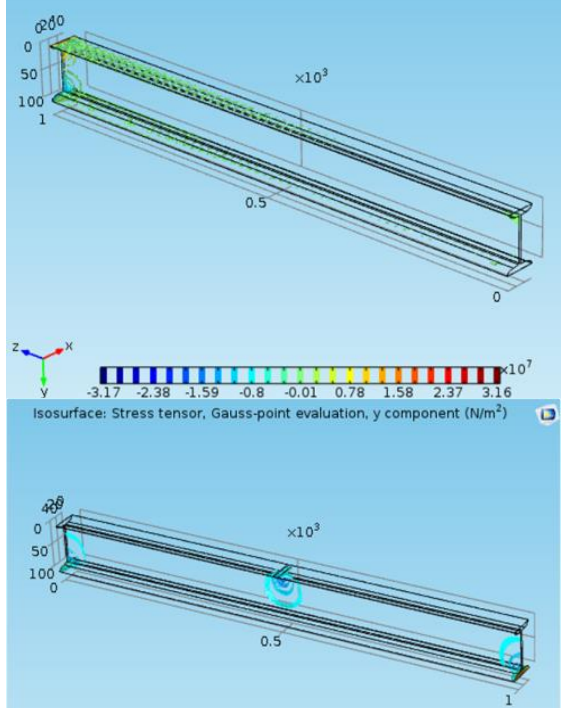

$\mathbf{H}$
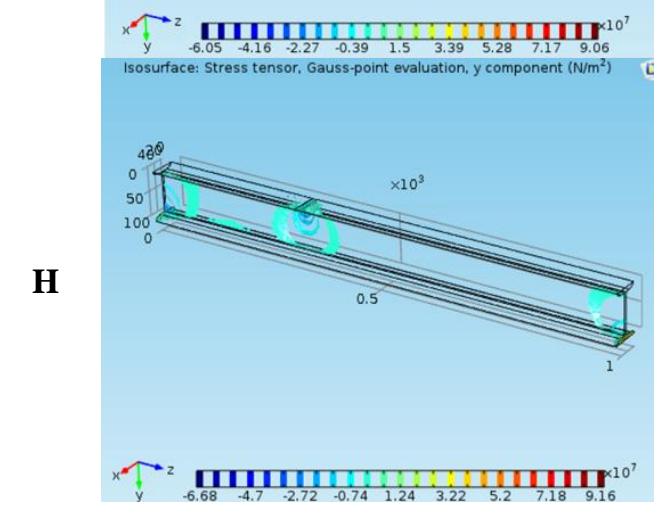

Figure 28 - Stress tensor, y component: A - the first scheme; $B$ - the second scheme; $C$ - the third scheme; D - the fourth scheme; $\mathbf{E}$ - the fifth scheme; $\mathbf{F}$ - the sixth scheme; $\mathbf{G}$ - the seventh scheme; $\mathrm{H}$ - the eighth scheme; I - the ninth scheme. 


\begin{tabular}{|c|c|c|c|c|c|c|}
\hline \multirow{4}{*}{ Impact Factor: } & ISRA (India) & $=3.117$ & SIS (USA) & $=0.912$ & ICV (Poland) & $=6.630$ \\
\hline & ISI (Dubai, UAE & $=0.829$ & РИНЦ (Russia & $=0.156$ & PIF (India) & $=1.940$ \\
\hline & GIF (Australia) & $=0.564$ & ESJI (KZ) & $=8.716$ & IBI (India) & $=4.260$ \\
\hline & JIF & $=1.500$ & SJIF (Morocco & $=5.667$ & OAJI (USA) & $=0.350$ \\
\hline
\end{tabular}

$\mathbf{A}$

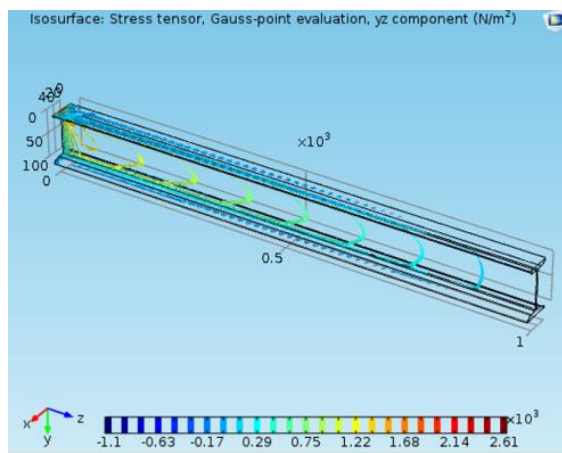

C
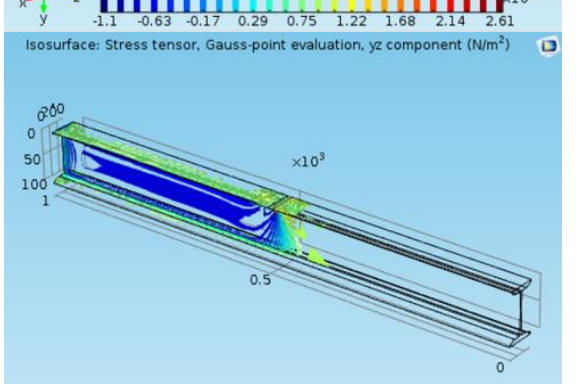

$\mathbf{E}$

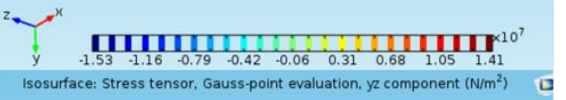

G
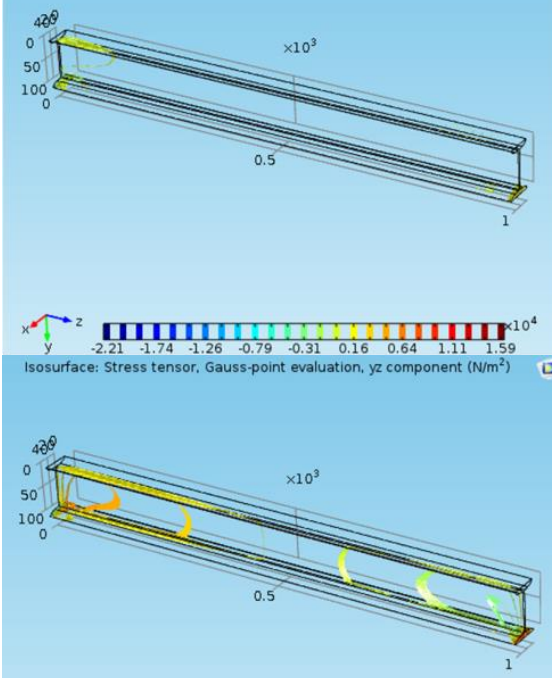

I

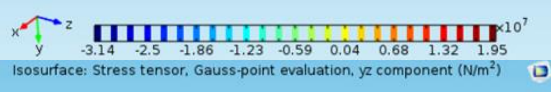

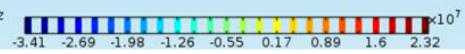

B

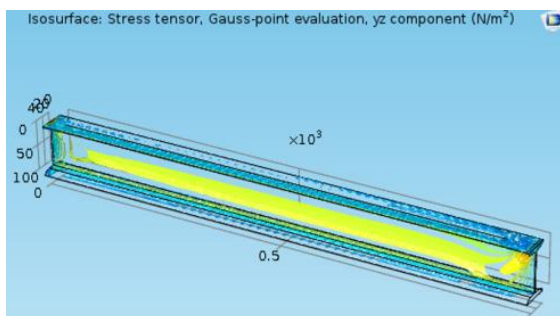

D

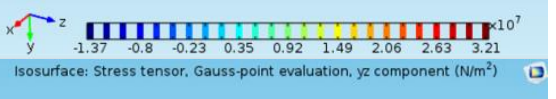

.0280

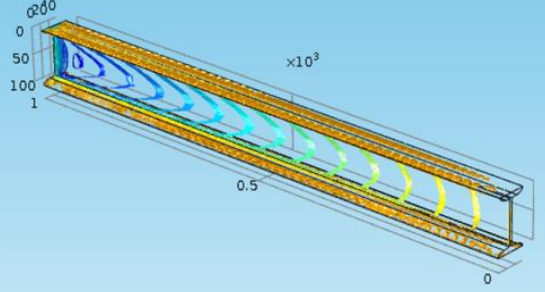

F

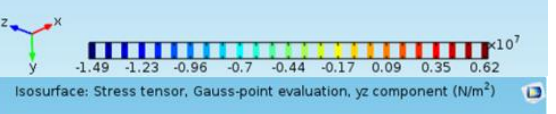

H
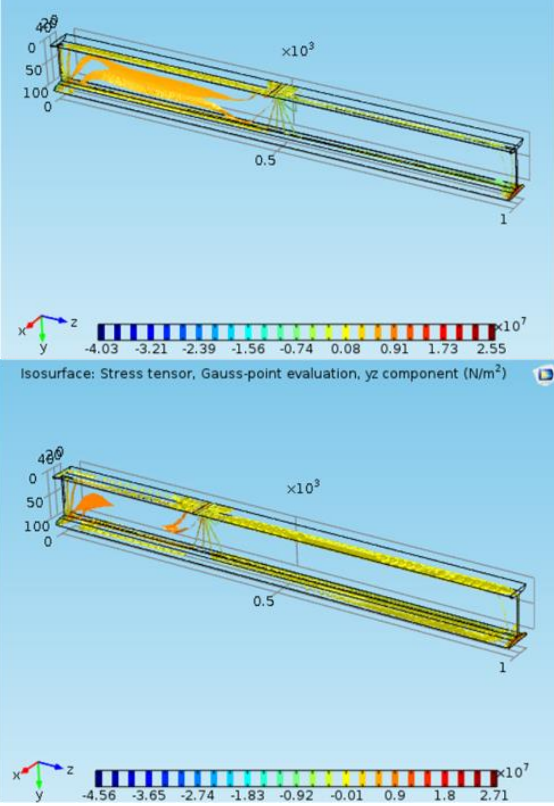

Figure 29 - Stress tensor, yz component: A - the first scheme; $B$ - the second scheme; $C$ - the third scheme; D - the fourth scheme; $\mathbf{E}$ - the fifth scheme; $\mathbf{F}$ - the sixth scheme; $\mathbf{G}$ - the seventh scheme; $\mathbf{H}$ - the eighth scheme; I - the ninth scheme. 


\begin{tabular}{|c|c|c|c|c|c|c|}
\hline \multirow{4}{*}{ Impact Factor: } & ISRA (India) & $=3.117$ & SIS (USA) & $=0.912$ & ICV (Poland) & $=6.630$ \\
\hline & ISI (Dubai, UAE & $=0.829$ & РИНЦ (Russia & $=0.156$ & PIF (India) & $=1.940$ \\
\hline & GIF (Australia) & $=0.564$ & ESJI (KZ) & $=8.716$ & IBI (India) & $=4.260$ \\
\hline & JIF & $=1.500$ & SJIF (Morocco & $=5.667$ & OAJI (USA) & $=0.350$ \\
\hline
\end{tabular}

A

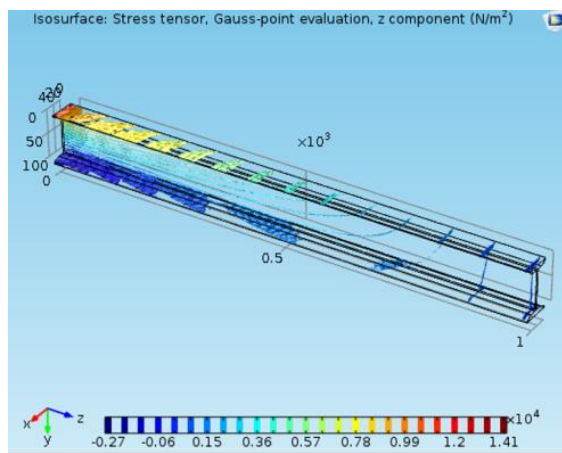

C

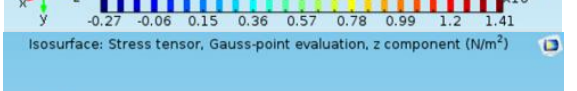

$\mathbf{E}$
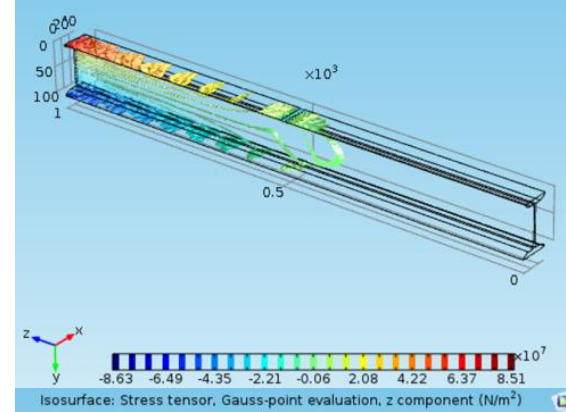

G

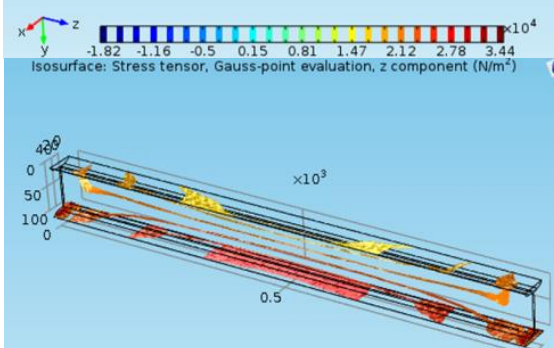

I

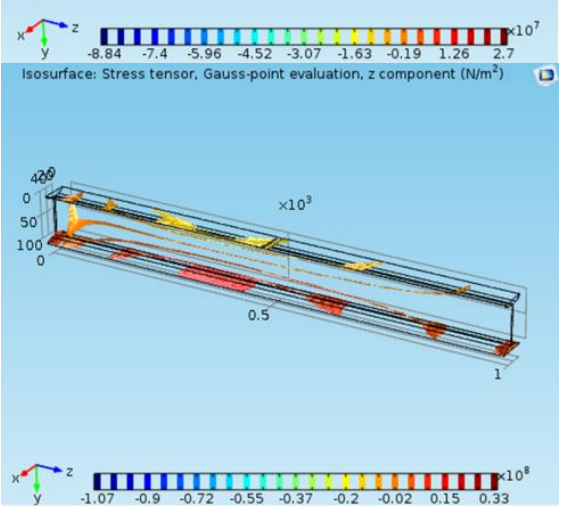

B

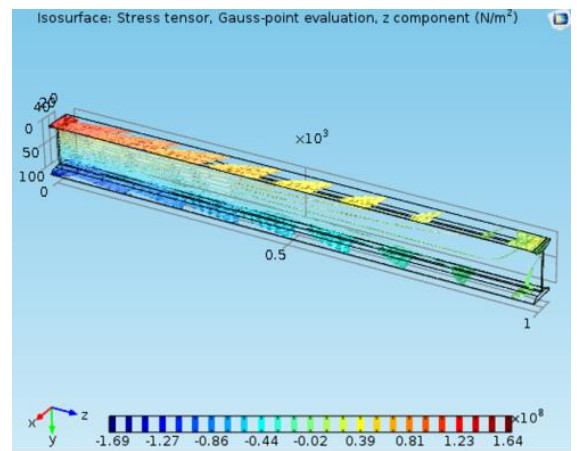

D
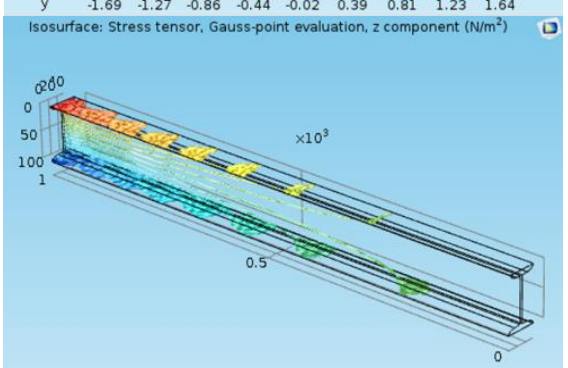

$\mathbf{F}$

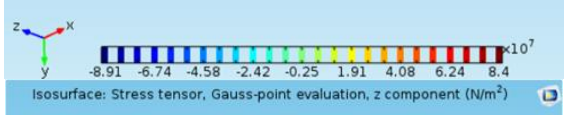

H

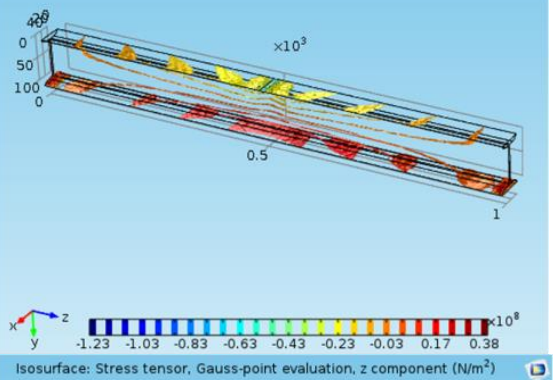

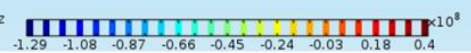

Figure 30 - Stress tensor, $\mathrm{z}$ component: A - the first scheme; B - the second scheme; C - the third scheme; D

- the fourth scheme; $\mathrm{E}$ - the fifth scheme; $\mathrm{F}$ - the sixth scheme; $\mathrm{G}$ - the seventh scheme; $\mathrm{H}$ - the eighth scheme; I - the ninth scheme. 


\begin{tabular}{|c|c|c|c|c|c|c|}
\hline \multirow{4}{*}{ Impact Factor: } & ISRA (India) & $=3.117$ & SIS (USA) & $=0.912$ & ICV (Poland) & $=6.630$ \\
\hline & ISI (Dubai, UAE & $=0.829$ & РИНЦ (Russia) & $=0.156$ & PIF (India) & $=1.940$ \\
\hline & GIF (Australia) & $=0.564$ & ESJI (KZ) & $=8.716$ & IBI (India) & $=4.260$ \\
\hline & JIF & $=1.500$ & SJIF (Morocco) & $=5.667$ & OAJI (USA) & $=0.350$ \\
\hline
\end{tabular}

$\mathbf{A}$

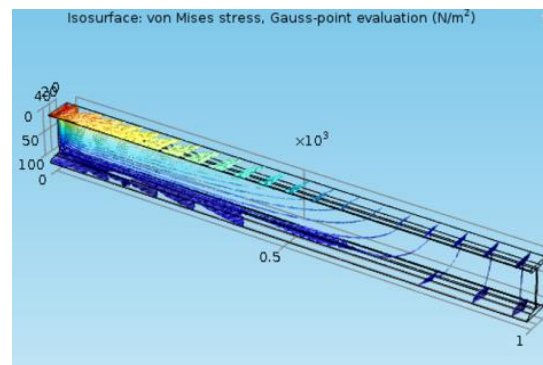

C

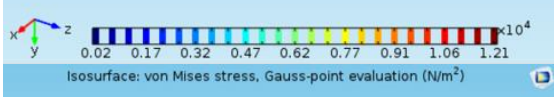

$\mathbf{E}$

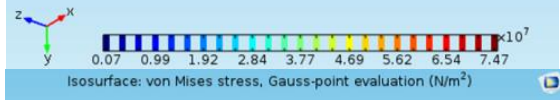

G

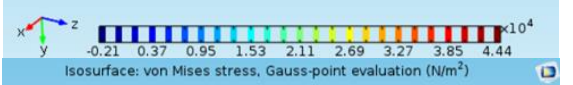

I
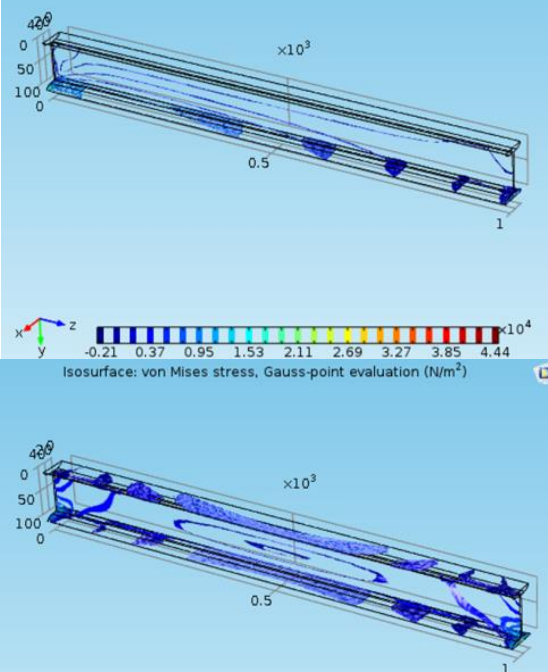

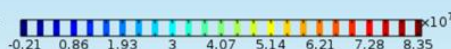

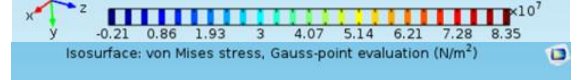

$\tau_{y}^{2} \operatorname{lmm}_{0.01} \min _{0.13} m_{0.38}$
B

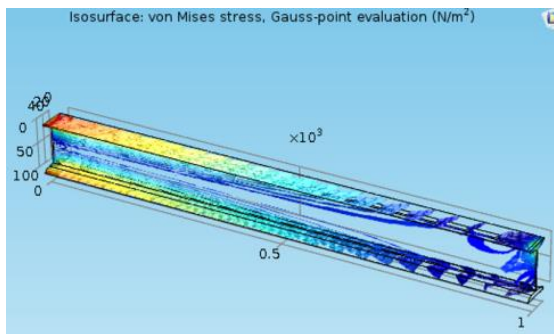

D

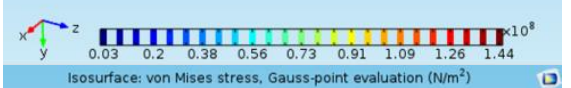

$\mathbf{F}$
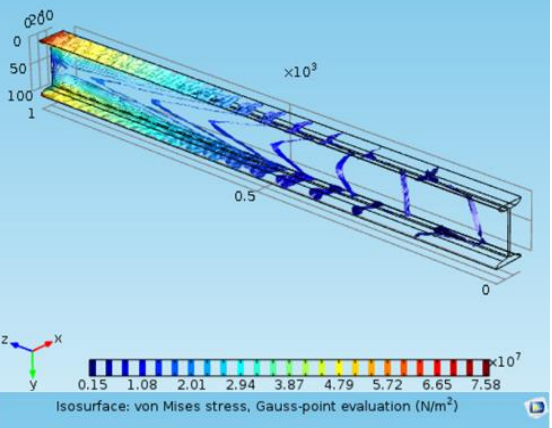

$\mathbf{H}$
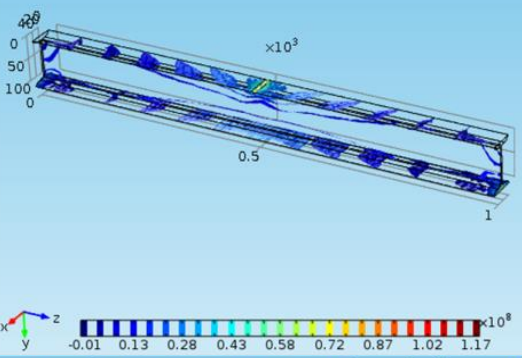

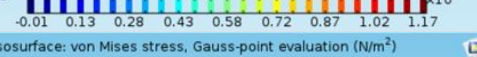

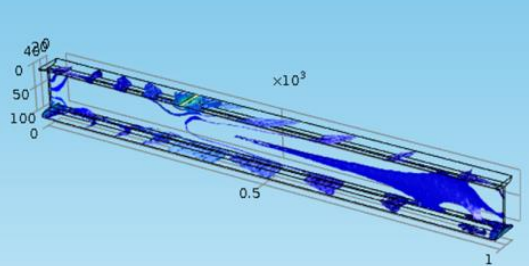

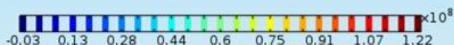

Figure 31 - Von Mises stress: A - the first scheme; B - the second scheme; C - the third scheme; D - the fourth scheme; E - the fifth scheme; F - the sixth scheme; G - the seventh scheme; H - the eighth scheme; I - the ninth scheme. 


\begin{tabular}{|c|c|c|c|c|c|c|}
\hline \multirow{4}{*}{ Impact Factor: } & ISRA (India) & $=3.117$ & SIS (USA) & $=0.912$ & ICV (Poland) & $=6.630$ \\
\hline & ISI (Dubai, UAE & $=0.829$ & РИНЦ (Russia & $=0.156$ & PIF (India) & $=1.940$ \\
\hline & GIF (Australia) & $=0.564$ & ESJI (KZ) & $=8.716$ & IBI (India) & $=4.260$ \\
\hline & JIF & $=1.500$ & SJIF (Morocco & $=5.667$ & OAJI (USA) & $=0.350$ \\
\hline
\end{tabular}

A

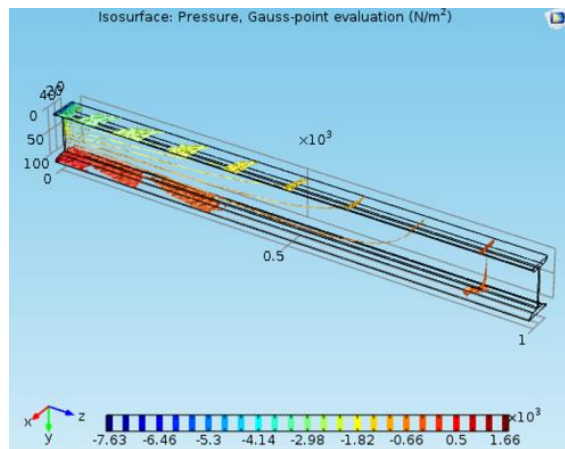

C

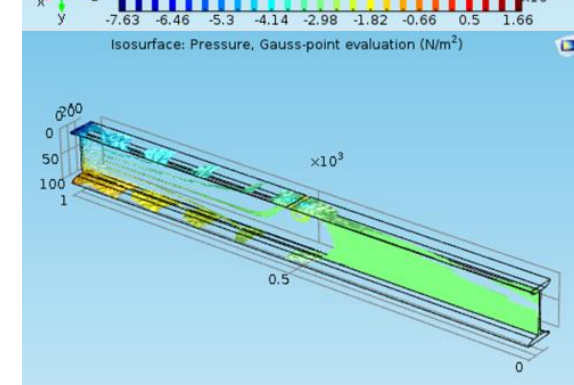

$\mathbf{E}$

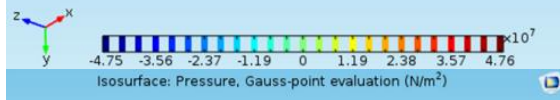

G

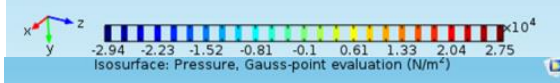

I
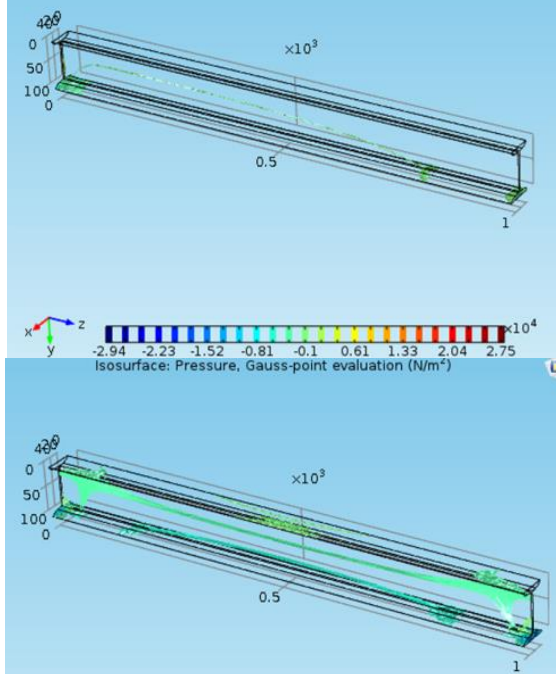

(1)

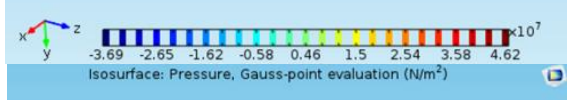

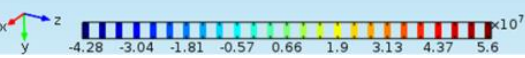

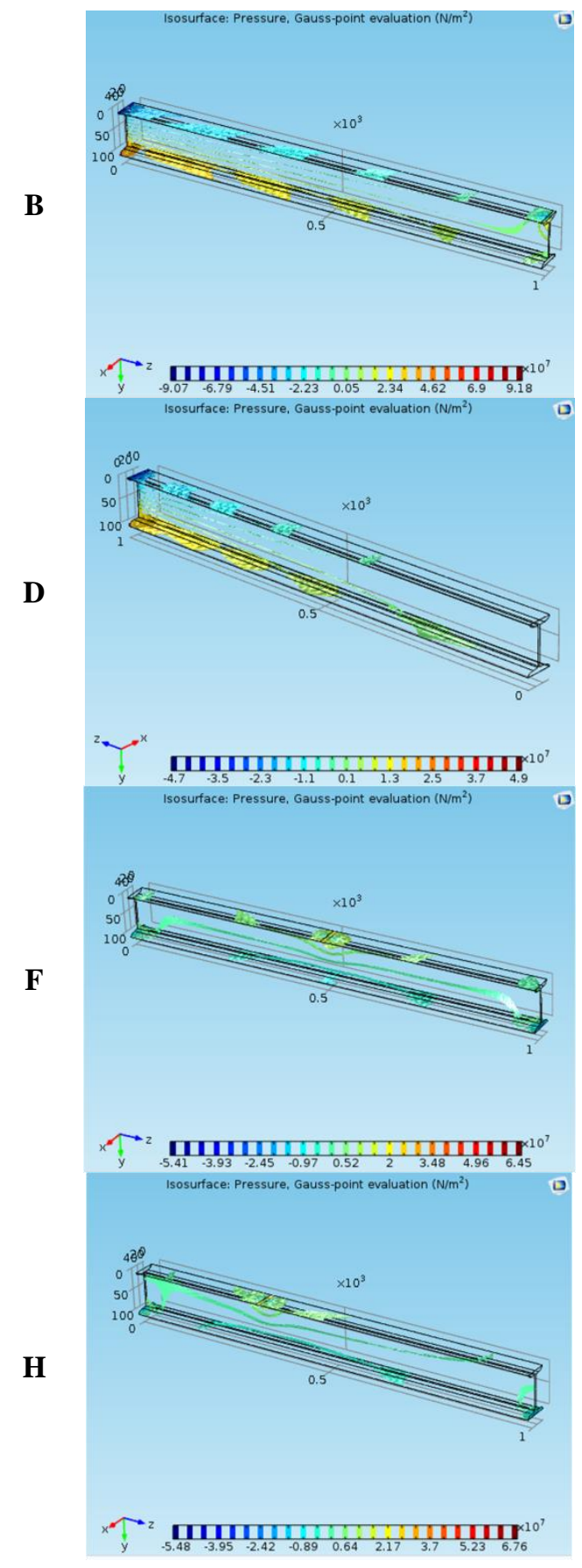

Figure 32 - Pressure: A - the first scheme; $\mathbf{B}$ - the second scheme; $\mathrm{C}$ - the third scheme; $\mathrm{D}$ - the fourth scheme; E - the fifth scheme; F - the sixth scheme; G - the seventh scheme; H - the eighth scheme; I - the ninth scheme. 


\begin{tabular}{|c|c|c|c|c|c|c|}
\hline \multirow{4}{*}{ Impact Factor: } & ISRA (India) & $=3.117$ & SIS (USA) & $=0.912$ & ICV (Poland) & $=6.630$ \\
\hline & ISI (Dubai, UAE & $=0.829$ & РИНЦ (Russia & $=0.156$ & PIF (India) & $=1.940$ \\
\hline & GIF (Australia) & $=0.564$ & ESJI (KZ) & $=8.716$ & IBI (India) & $=4.260$ \\
\hline & JIF & $=1.500$ & SJIF (Morocco & $=5.667$ & OAJI (USA) & $=0.350$ \\
\hline
\end{tabular}

$\mathbf{A}$

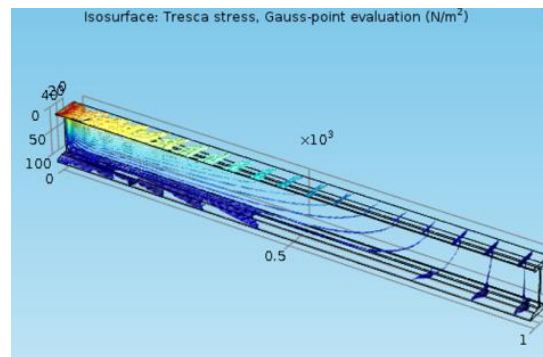

C

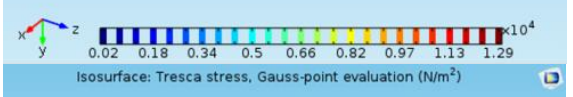

$\mathbf{E}$
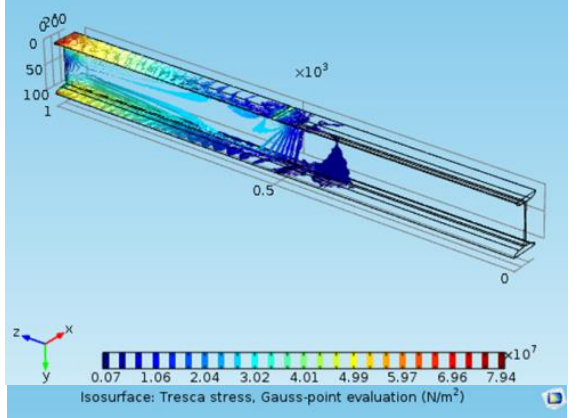

G

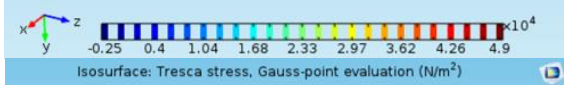

I

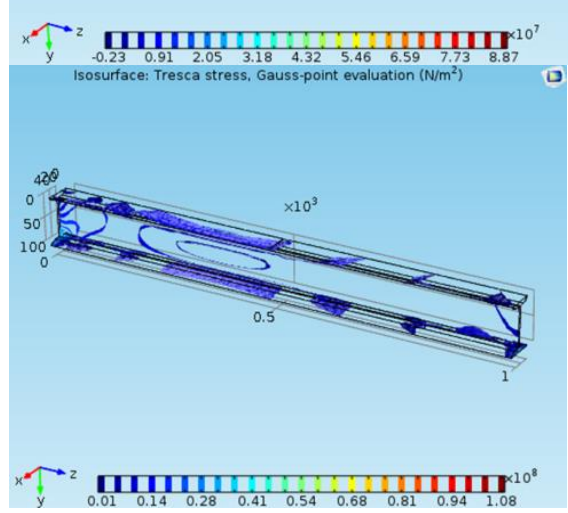

B

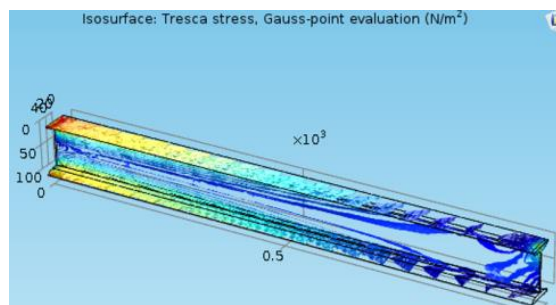

D
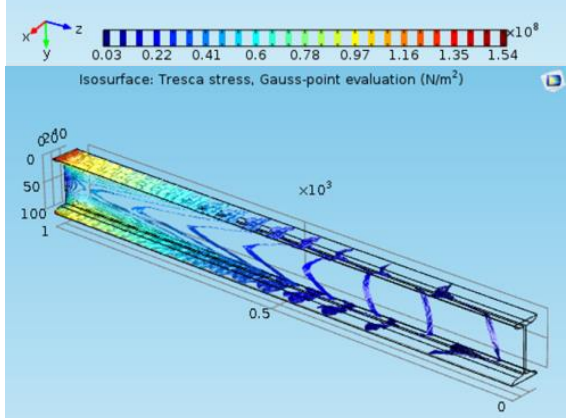

$\mathbf{F}$

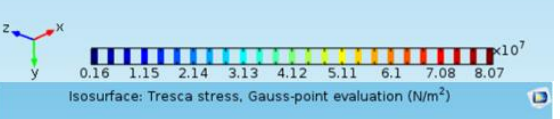

$\mathbf{H}$
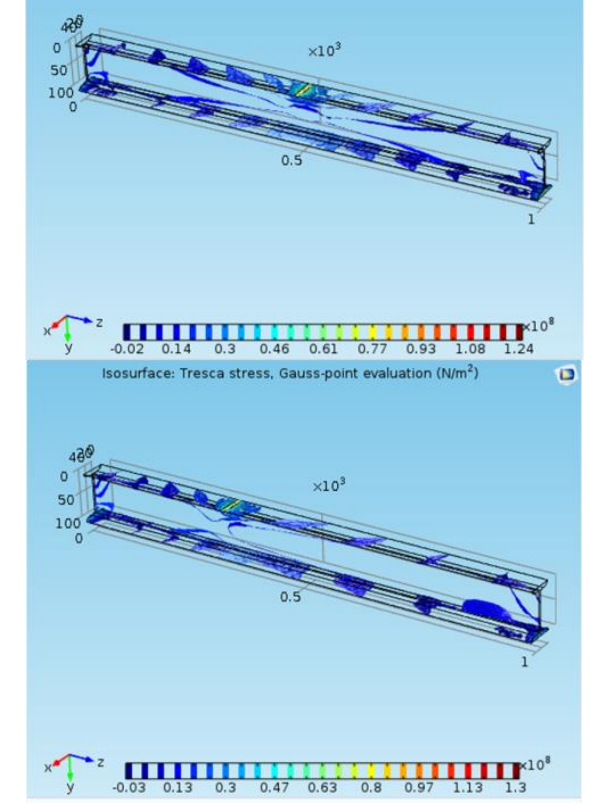

Figure 33 - Tresca stress: A - the first scheme; B - the second scheme; C - the third scheme; D - the fourth scheme; $\mathrm{E}$ - the fifth scheme; $\mathrm{F}$ - the sixth scheme; $\mathrm{G}$ - the seventh scheme; $\mathrm{H}$ - the eighth scheme; I - the ninth scheme. 


\begin{tabular}{|c|c|c|c|c|c|c|}
\hline \multirow{4}{*}{ Impact Factor: } & ISRA (India) & $=3.117$ & SIS (USA) & $=0.912$ & ICV (Poland) & $=6.630$ \\
\hline & ISI (Dubai, UAE & $=0.829$ & РИНЦ (Russia) & $=0.156$ & PIF (India) & $=1.940$ \\
\hline & GIF (Australia) & $=0.564$ & ESJI (KZ) & $=8.716$ & IBI (India) & $=4.260$ \\
\hline & JIF & $=1.500$ & SJIF (Morocco) & $=5.667$ & OAJI (USA) & $=0.350$ \\
\hline
\end{tabular}

A

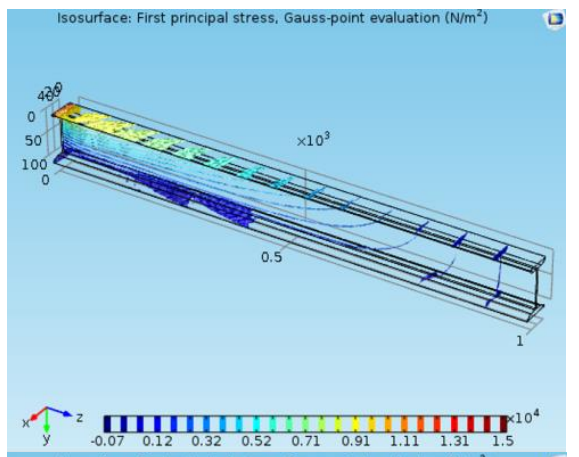

C

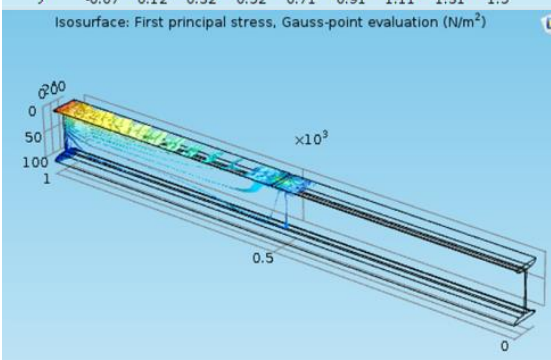

$\mathbf{E}$

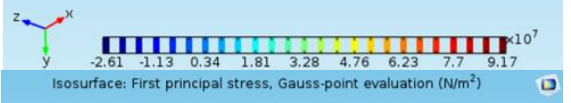

G
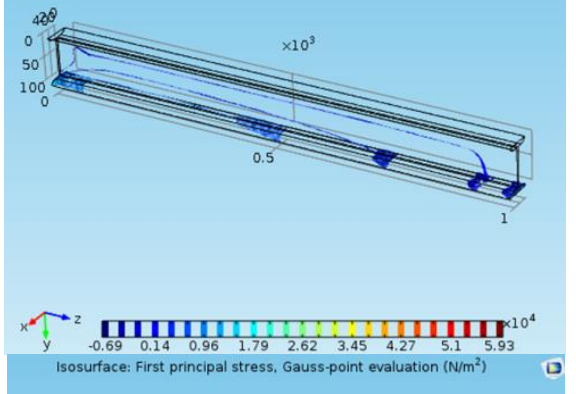

I

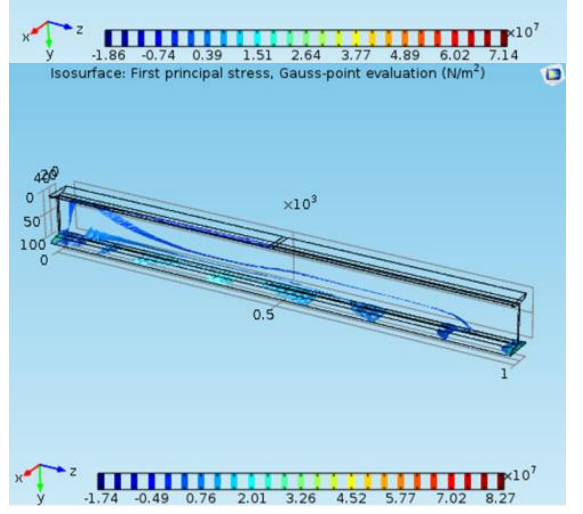

B

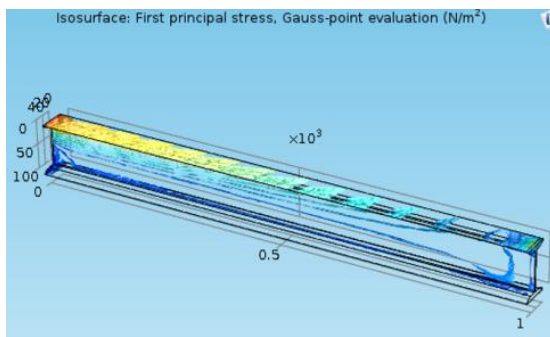

D
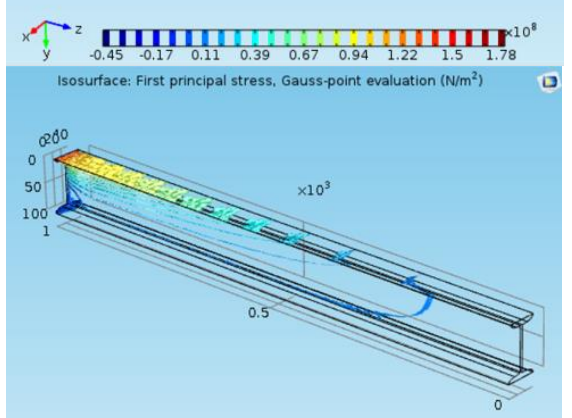

$\mathbf{F}$
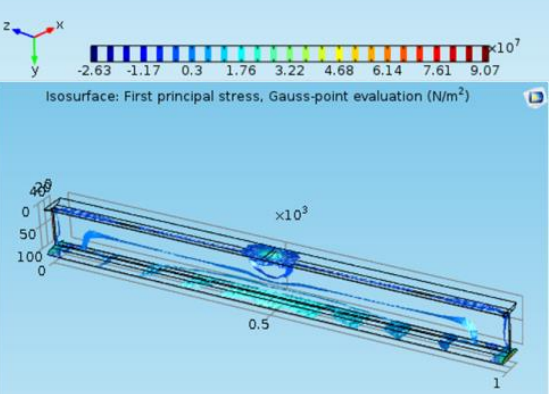

$\mathbf{H}$

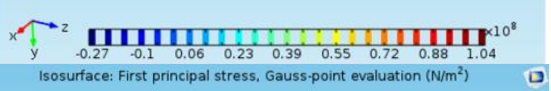

$$
\text { H }
$$

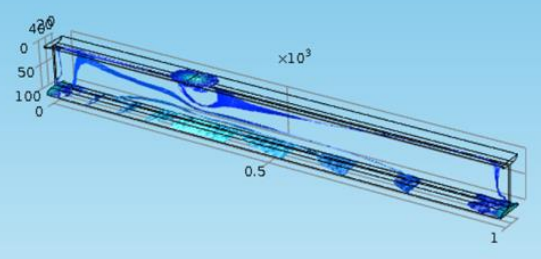

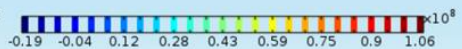

Figure 34 - First principal stress: A - the first scheme; B - the second scheme; C - the third scheme; D - the fourth scheme; $\mathbf{E}$ - the fifth scheme; $\mathbf{F}$ - the sixth scheme; $\mathbf{G}$ - the seventh scheme; H - the eighth scheme; I - the ninth scheme. 


\begin{tabular}{|c|c|c|c|c|c|c|}
\hline \multirow{4}{*}{ Impact Factor: } & ISRA (India) & $=3.117$ & SIS (USA) & $=0.912$ & ICV (Poland) & $=6.630$ \\
\hline & ISI (Dubai, UAE & $=0.829$ & РИНЦ (Russia & $=0.156$ & PIF (India) & $=1.940$ \\
\hline & GIF (Australia) & $=0.564$ & ESJI (KZ) & $=8.716$ & IBI (India) & $=4.260$ \\
\hline & JIF & $=1.500$ & SJIF (Morocco & $=5.667$ & OAJI (USA) & $=0.350$ \\
\hline
\end{tabular}

A

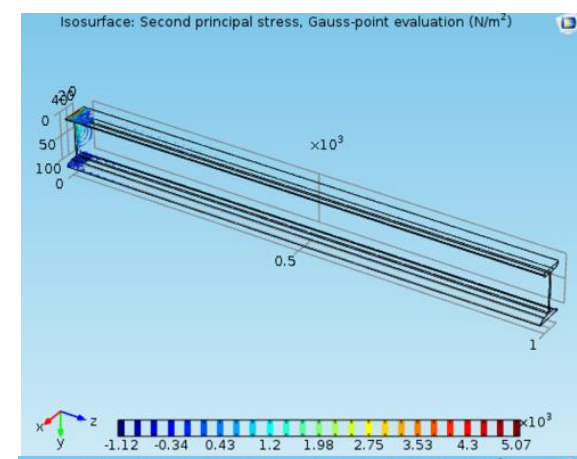

C

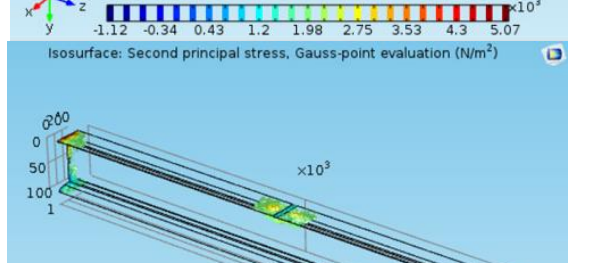

E

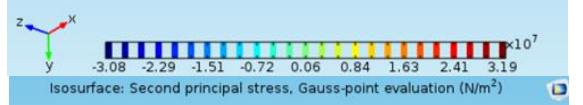

G
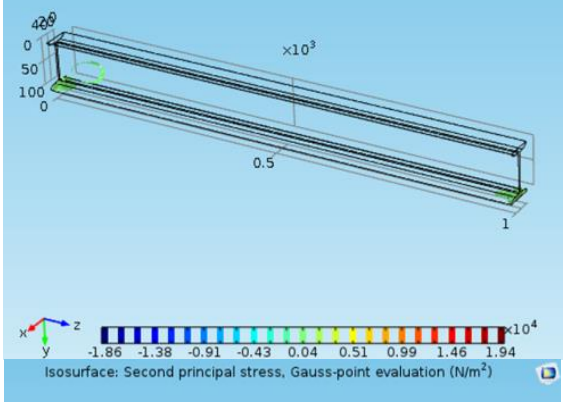

I

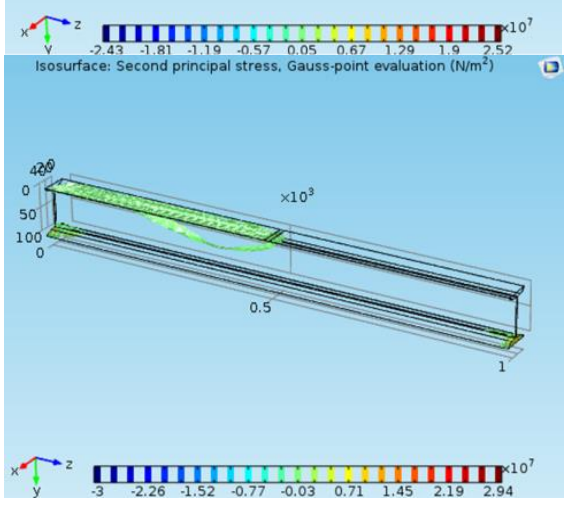

B

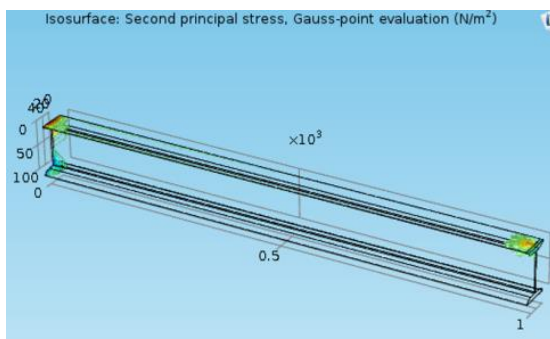

D

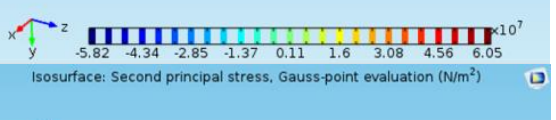

F
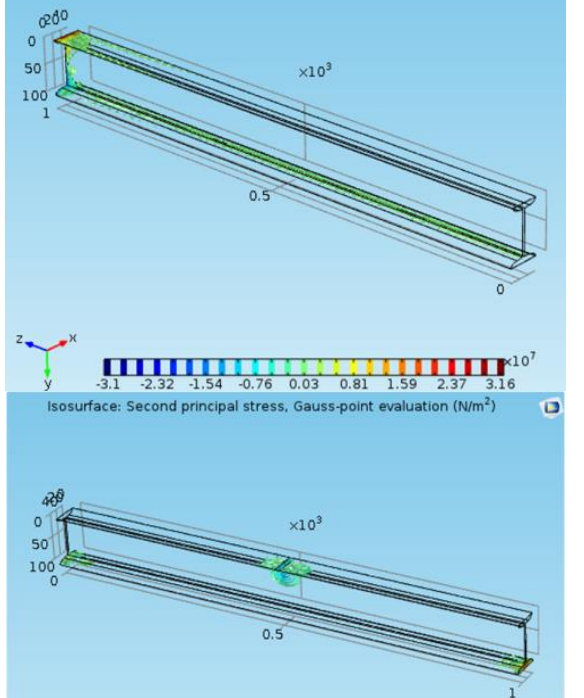

H
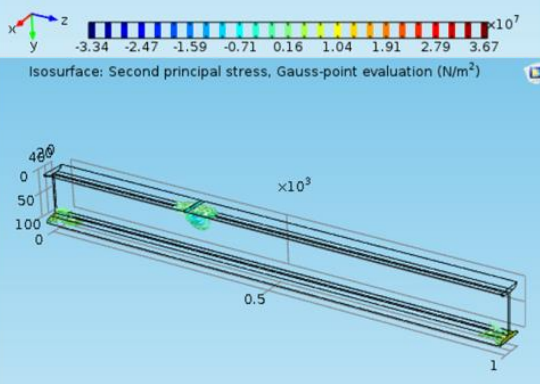

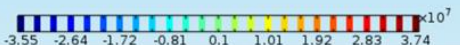

Figure 35 - Second principal stress: A - the first scheme; $B$ - the second scheme; $C$ - the third scheme; D the fourth scheme; E - the fifth scheme; F - the sixth scheme; G - the seventh scheme; H - the eighth scheme; I - the ninth scheme. 


\begin{tabular}{|c|c|c|c|c|c|c|}
\hline \multirow{4}{*}{ Impact Factor: } & ISRA (India) & $=3.117$ & SIS (USA) & $=0.912$ & ICV (Poland) & $=6.630$ \\
\hline & ISI (Dubai, UAE & $=0.829$ & РИНЦ (Russia & $=0.156$ & PIF (India) & $=1.940$ \\
\hline & GIF (Australia) & $=0.564$ & ESJI (KZ) & $=8.716$ & IBI (India) & $=4.260$ \\
\hline & JIF & $=1.500$ & SJIF (Morocco & $=5.667$ & OAJI (USA) & $=0.350$ \\
\hline
\end{tabular}

$\mathbf{A}$

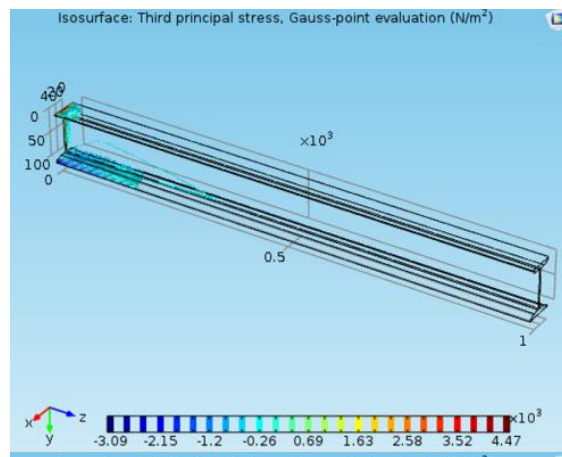

C

$\mathbf{E}$

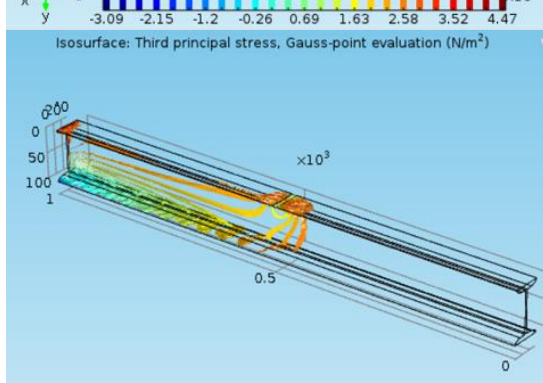

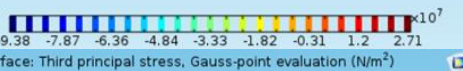

G

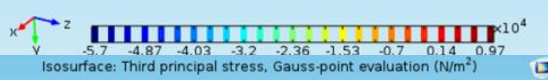

I
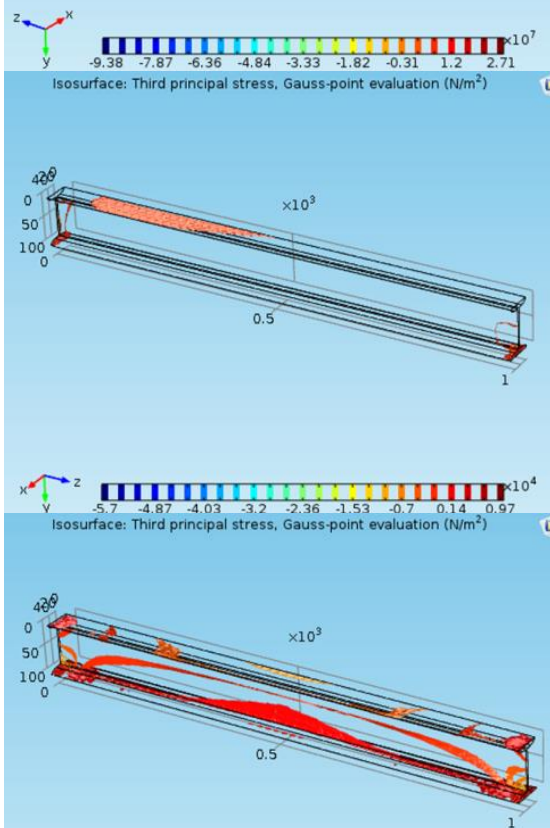

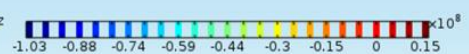

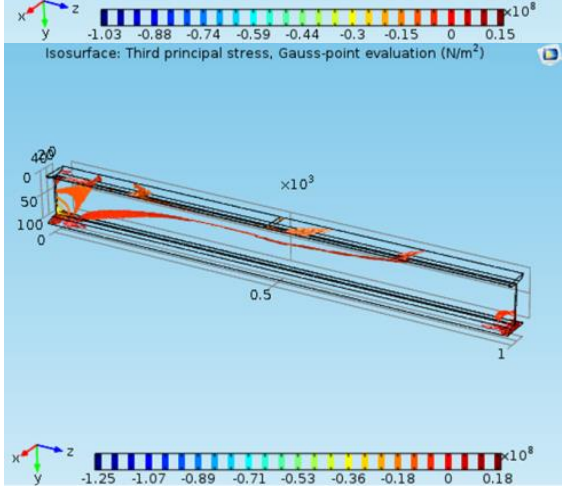

B

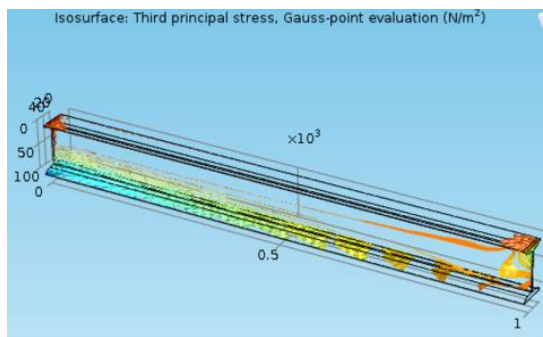

D
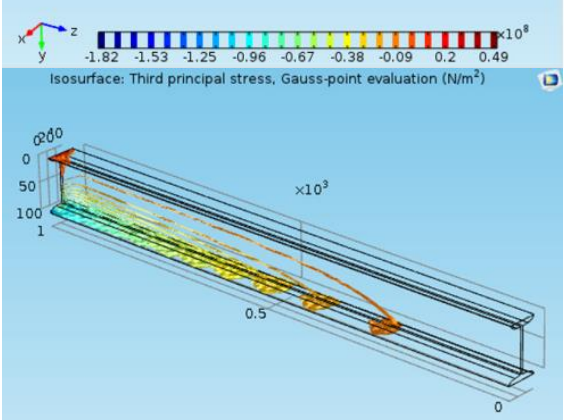

F

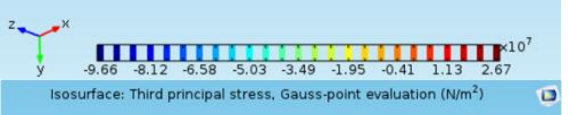

H
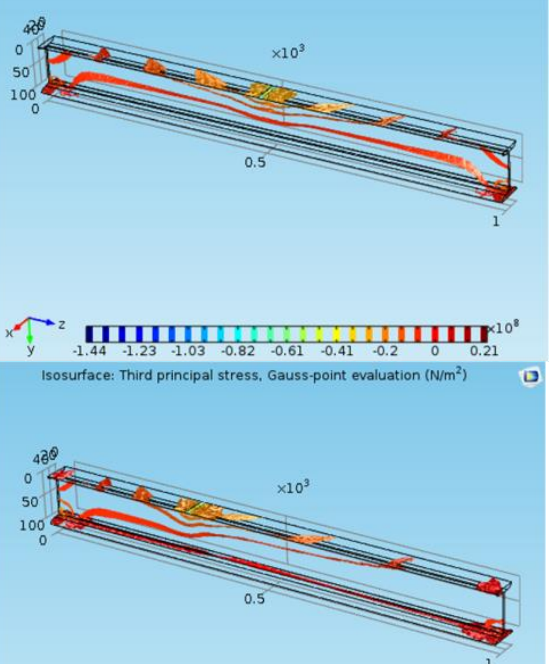

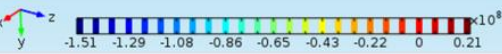

Figure 36 - Third principal stress: A - the first scheme; B - the second scheme; C - the third scheme; D the fourth scheme; $\mathrm{E}$ - the fifth scheme; $\mathrm{F}$ - the sixth scheme; $\mathrm{G}$ - the seventh scheme; $\mathrm{H}$ - the eighth scheme; I - the ninth scheme. 


\begin{tabular}{|c|c|c|c|c|c|c|}
\hline \multirow{4}{*}{ Impact Factor: } & ISRA (India) & $=3.117$ & SIS (USA) & $=0.912$ & ICV (Poland) & $=6.630$ \\
\hline & ISI (Dubai, UAE & $=0.829$ & РИНЦ (Russia & $=0.156$ & PIF (India) & $=1.940$ \\
\hline & GIF (Australia) & $=0.564$ & ESJI (KZ) & $=8.716$ & IBI (India) & $=4.260$ \\
\hline & JIF & $=1.500$ & SJIF (Morocco & $=5.667$ & OAJI (USA) & $=0.350$ \\
\hline
\end{tabular}

Von Mises stress is equivalent stress defined as the sum of all multidirectional stresses of material. Maximum von Mises stress of the cantilever I-beams is concentrated in cross sections of the elements close to the supports. Loading of the doubly supported I-beams by forces and moment is characterized by uniform distribution of the values of von Mises stress. The isosurfaces of von Mises stress in the inner layers have the elliptic shape at action of distributed force on the doubly supported I-beam.

The pressure isosurfaces characterize a stress type of the I-beams material. In the cantilever Ibeams above the neutral axis there is tensile deformation, below the neutral axis there is compressive deformation. Tensile deformation (in the inner layers of material) prevails in the doubly supported I-beams.

Tresca stress is shear stress of material. It is introduced the criterion of maximum shear stress at which there is material destruction. The isosurfaces distribution of Tresca stress is identical to the isosurfaces distribution of von Mises stress. The average ratio of Tresca stress to von Mises stress is 1.065 .

Normal stresses on areas of the selected element with zero shear stresses are called principal. Maximum and minimum principal stresses are stresses acting perpendicular to the plane on which shear stress is zero. Maximum principal stress allows to determine maximum tensile stress that occurs in the structural element due to the load conditions. Minimum principal stress allows to determine maximum compressive stress that occurs in the structural element due to the load conditions. The isosurfaces of the first and third principal stresses allow to identify the dangerous sections of the Ibeams. The volumes of the top flange at the distance from rigid restraint to middle of the span length are the dangerous sections for the second loading scheme. The isosurfaces of the second principal stress show local stresses of material in the application place of concentrated or distributed forces, as well as moment.

\section{Conclusion}

Action of concentrated force on the loose end of the cantilever I-beam can lead to significant plastic deformations of material. Material of the cantilever and doubly supported I-beams is subjected to stress more along the $Z$-axis. Stress distribution of the doubly supported I-beams material to the left and the right of the application place of concentrated and distributed forces is symmetrical. The most complex stress condition of material was determined at shortterm loading of the doubly supported I-beams (variable stress of the web material).

\section{References:}

1. Andjelic, N., \& Milosevic-Mitic, V. (2012). Optimum design of thin-walled I-beam subjected to stress constraint. Journal of Theoretical and Applied Mechanics, 50(4), 987999.

2. Cejpek, J., \& Juračka, J. (2016). Modifications of a simple I-beam and its effects on the stress state. Aviation, 20, 4, 168-172.

3. Moorman, R. B. B. (1937). Stresses in the curved beam under loads normal to the plane of its axis. Retrospective Theses and Dissertations, 14122.

4. Patel, R., Dubey, S. K., \& Pathak, K. K. (2014). Effect of depth span ratio on the behaviour of beams. International Journal of Advanced Structural Engineering, 6:3.

5. Patil, T., \& Shelke, N. L. (2016). Structural Analysis of a Cantilever Beam with Tapered Web Section through Fea. J. Steel Struct. Constr., 2:119.
6. Kim, B., Oliver, A., \& Vyse, A. (2013). Bending stresses of steel web tapered tee section cantilevers. Journal of Civil Engineering and Architecture, 7, 1329-1342.

7. Denan, F., Osman, M. H., \& Saad, S. (2010). The study of lateral torsional buckling behaviour of a beam with trapezoid web steel section by experimental and finite element analysis. International Journal of Research and Reviews in Applied Sciences, 2, 232-240.

8. Ashby, M. F. (2005). Materials Selection in Mechanical Design, Elsevier.

9. Chemezov, D., Osipov, T., \& Pesenko, A. (2016). A static calculation of an I-beam. ISJ Theoretical \& Applied Science, 11 (43), 49-52.

10. Chemezov, D., et al. (2019). Bendings of cantilever and doubly supported steel I-beams. ISJ Theoretical \& Applied Science, 01 (69), 261-267. 